NBER WORKING PAPER SERIES

\title{
BEST LINEAR APPROXIMATIONS TO SET IDENTIFIED FUNCTIONS: WITH AN APPLICATION TO THE GENDER WAGE GAP
}

\author{
Arun G. Chandrasekhar \\ Victor Chernozhukov \\ Francesca Molinari \\ Paul Schrimpf \\ Working Paper 25593 \\ http://www.nber.org/papers/w25593 \\ NATIONAL BUREAU OF ECONOMIC RESEARCH \\ 1050 Massachusetts Avenue \\ Cambridge, MA 02138 \\ February 2019
}

We are grateful to Casey Mulligan and Yona Rubinstein for sharing their data, and Denis Chetverikov and Hiroaki Kaido for comments. We thank seminar participants at Bern, Collegio Carlo Alberto, Penn State, Toronto, Toulouse, UCL, Yale, the 2012 Winter Meetings of the Econometric Society, and the 2013 Cowles Econometrics Conference for comments. Financial support from the NSF through grants SES-0922330 and SES-1824448 (Molinari) is gratefully acknowledged. Chernozhukov acknowledges NSF support. The views expressed herein are those of the authors and do not necessarily reflect the views of the National Science Foundation and the National Bureau of Economic Research.

NBER working papers are circulated for discussion and comment purposes. They have not been peer-reviewed or been subject to the review by the NBER Board of Directors that accompanies official NBER publications.

(C) 2019 by Arun G. Chandrasekhar, Victor Chernozhukov, Francesca Molinari, and Paul Schrimpf. All rights reserved. Short sections of text, not to exceed two paragraphs, may be quoted without explicit permission provided that full credit, including $\odot$ notice, is given to the source. 
Best Linear Approximations to Set Identified Functions: With an Application to the Gender Wage Gap Arun G. Chandrasekhar, Victor Chernozhukov, Francesca Molinari, and Paul Schrimpf NBER Working Paper No. 25593

February 2019

JEL No. C13,C31

\section{ABSTRACT}

This paper provides inference methods for best linear approximations to functions which are known to lie within a band. It extends the partial identification literature by allowing the upper and lower functions defining the band to carry an index, and to be unknown but parametrically or non-parametrically estimable functions. The identification region of the parameters of the best linear approximation is characterized via its support function, and limit theory is developed for the latter. We prove that the support function can be approximated by a Gaussian process and establish validity of the Bayesian bootstrap for inference. Because the bounds may carry an index, the approach covers many canonical examples in the partial identification literature arising in the presence of interval valued outcome and/or regressor data: not only mean regression, but also quantile and distribution regression, including sample selection problems, as well as mean, quantile, and distribution treatment effects. In addition, the framework can account for the availability of instruments. An application is carried out, studying female labor force participation using data from Mulligan and Rubinstein (2008) and insights from Blundell, Gosling, Ichimura, and Meghir (2007). Our results yield robust evidence of a gender wage gap, both in the 1970s and 1990s, at quantiles of the wage distribution up to the 0.4, while allowing for completely unrestricted selection into the labor force. Under the assumption that the median wage offer of the employed is larger than that of individuals that do not work, the evidence of a gender wage gap extends to quantiles up to the 0.7. When the assumption is further strengthened to require stochastic dominance, the evidence of a gender wage gap extends to all quantiles, and there is some evidence at the 0.8 and higher quantiles that the gender wage gap decreased between the 1970s and 1990s.

Arun G. Chandrasekhar

Department of Economics

Stanford University

579 Serra Mall

Stanford, CA 94305

and NBER

arungc@stanford.edu

Victor Chernozhukov

Department of Economics

Massachusetts Institute of Technology

77 Massachusetts Avenue Cambridge,

Mass. 02139

vchern@mit.edu
Francesca Molinari

Department of Economics

Cornell University

Ithaca, NY

fm72@cornell.edu

Paul Schrimpf

Vancouver School of Economics

University of British Columbia

997 - 1873 East Mall Vancouver,

BC V6T1Z1

schrimpf@mail.ubc.ca 


\section{INTRODUCTION}

This paper contributes to the partial identification literature by providing estimation and inference results for best linear approximations to set identified functions. Specifically, we work with a family of functions $f(x, \alpha)$ indexed by some parameter $\alpha \in \mathcal{A}$, that is known to satisfy $\theta_{0}(x, \alpha) \leq f(x, \alpha) \leq \theta_{1}(x, \alpha), x-a . s$., with $x \in \mathbb{R}^{d}$ a vector of covariates. Econometric frameworks yielding such restriction are ubiquitous in economics and in the social sciences, as illustrated by Manski $(2003,2007)$. Cases explicitly analyzed in this paper include: (1) mean regression; (2) quantile regression; and (3) distribution and duration regression, in the presence of interval valued outcome and/or covariate data, including hazard models with interval-valued failure times; (4) sample selection problems; (5) mean treatment effects; (6) quantile treatment effects; and (7) distribution treatment effects, see Section 3 for details 11 Yet, the methodology that we propose can be applied to virtually any of the frameworks discussed in Manski $(2003,2007)$. In fact, our results below also allow for exclusion restrictions that yield intersection bounds of the form $\sup _{v \in \mathcal{V}} \theta_{0}(x, v, \alpha) \equiv$ $\theta_{0}(x, \alpha) \leq f(x, \alpha) \leq \theta_{1}(x, \alpha) \equiv \inf _{v \in \mathcal{V}} \theta_{1}(x, v, \alpha) x-a . s$., with $v$ an instrumental variable taking values in a finite set $\mathcal{V}$. The bounding functions $\theta_{0}(x, \alpha)$ and $\theta_{1}(x, \alpha)$ may be indexed by a parameter $\alpha \in \mathcal{A}$ and may be any estimable function of $x$.

When the restriction $\theta_{0}(x, \alpha) \leq f(x, \alpha) \leq \theta_{1}(x, \alpha) x$-a.s. summarizes all the information available to the researcher, the identification region for $f(\cdot, \alpha)$ is given by the set of functions

$$
\mathfrak{F}(\alpha)=\left\{\phi(\cdot, \alpha): \theta_{0}(x, \alpha) \leq \phi(x, \alpha) \leq \theta_{1}(x, \alpha) x-a . s .\right\}
$$

The set $\mathfrak{F}(\alpha)$, while sharp, can be difficult to interpret and report, especially when $x$ is multi-dimensional. Similar considerations apply to related sets, e.g. the set of marginal effects of components of $x$ on $f(x, \alpha)$. Consequently, in this paper we focus on the sharp set of parameters characterizing best linear approximations to the functions comprising $\mathfrak{F}(\alpha)$. This set, denoted $B(\alpha)$ in what follows, is of great interest in empirical work because of its tractability. In particular, it can be computed extremely rapidly using standard statistical packages such as Stata or $\mathrm{R} \mathrm{L}^{2}$

Our method appears to be the first and currently only method available in the literature for performing inference on the set $B(\alpha)$ and its elements when the bounding functions $\theta_{0}(\cdot, \alpha)$ and $\theta_{1}(\cdot, \alpha)$ need to be estimated. This estimation may be carried out both parametrically as well as non-parametrically via series methods. Previous closely related contributions by Beresteanu and Molinari (2008, BM henceforth) and Bontemps, Magnac, and Maurin (2012) provided inference methods for best linear approximations to conditional expectations in the presence of interval outcome data. In that environment, the bounding functions do not need to be estimated, as the set of best linear approximations can be

\footnotetext{
${ }^{1}$ For example, one may be interested in the $\alpha$-conditional quantile of a random variable $y$ given $x$, denoted $Q_{y}(\alpha \mid x)$, but only observe interval data $\left[y_{0}, y_{1}\right]$ which contain $y$ with probability one. In this case, $f(x, \alpha) \equiv Q_{y}(\alpha \mid x)$ and $\theta_{\ell}(x, \alpha) \equiv Q_{\ell}(\alpha \mid x), \ell=0,1$, the conditional quantiles of properly specified random variables.

${ }^{2}$ An implementation in $\mathrm{R}$ with non-parametrically estimated functions $\theta_{0}(\cdot, \alpha), \theta_{1}(\cdot, \alpha)$ can be found at https://bitbucket.org/paulschrimpf/mulligan-rubinstein-bounds. An implementation in Stata with observed $\theta_{0}(\cdot, \alpha), \theta_{1}(\cdot, \alpha)$ can be found at https://molinari.economics.cornell.edu/programs/Stata_ SetBLP.zip
} 
characterized directly through functions of moments of the observable variables. Hence, our paper builds upon and significantly generalizes their results. These generalizations are our main contribution and are important for many empirically relevant applications.

As we discuss in Section 2 the set $B(\alpha)$ is convex and hence can be fully characterized by its support function $\left.\right|^{3}$ We therefore use this function to carry out inference for $B(\alpha)$ and its elements. An application of results in BM and Bontemps, Magnac, and Maurin (2012) gives that the support function of $B(\alpha)$ is equal to the expectation of a function $s\left(\theta_{0}(x, \alpha), \theta_{1}(x, \alpha), x, \mathrm{E}\left(x x^{\prime}\right)\right)$. Hence, the analogy principle suggests to estimate the support function of $B(\alpha)$ through a sample average of the same function $s$, where $\theta_{0}(x, \alpha)$ and $\theta_{1}(x, \alpha)$ are replaced by parametric or non-parametric estimators, and $\mathrm{E}\left(x x^{\prime}\right)$ is replaced by its sample analog. We show that the resulting estimator is consistent for the support function of $B(\alpha)$, uniformly over $(q, \alpha) \in \mathcal{S}^{d-1} \times \mathcal{A}$, where $\mathcal{S}^{d-1}:=\left\{q \in \mathbb{R}^{d}:\|q\|=1\right\}$ is the range of directions for which the support function needs to be evaluated to fully characterize $B(\alpha)$. We then provide a methodology for inference. In doing so, our paper overcomes significant technical complications, thereby making contributions of independent interest.

First, when $\theta_{0}(\cdot, \alpha)$ and $\theta_{1}(\cdot, \alpha)$ are non-parametrically estimated through series methods, we show that the support function process is strongly approximated by a Gaussian process on $\mathcal{S}^{d-1} \times \mathcal{A}$ that may not necessarily converge as the number of series functions increases to infinity. To solve this difficulty, we show that each subsequence has a further subsequence converging to a tight Gaussian process on $\mathcal{S}^{d-1} \times \mathcal{A}$ with a uniformly equicontinuous and non-degenerate covariance function. We show how to conduct inference using properties of this covariance function, and we provide a consistent Bayesian bootstrap procedure to estimate quantiles of functions of the Gaussian process.

Second, we allow for the possibility that some of the regressors in $x$ have a discrete distribution. In order to conduct test of hypothesis and make confidence statements, both BM and Bontemps, Magnac, and Maurin (2012) had explicitly ruled out discrete regressors, as their presence greatly complicates the derivation of the limiting distribution of the support function process. By using a simple data-jittering technique, we show that these complications completely disappear, albeit at the cost of basing statistical inference on a slightly conservative confidence set 4

Third the function $f(\cdot, \alpha)$ may be set identified via intersection of bounds of the form $\sup _{v \in \mathcal{V}} \theta_{0}(x, v, \alpha) \leq f(x, \alpha) \leq \inf _{v \in \mathcal{V}} \theta_{1}(x, v, \alpha) x-a . s$., with $\mathcal{V}$ a finite set. In this case we show that the set of best linear approximations to the intersected bands of functions is equal to the intersection of the sets of best linear approximations obtained for each $v \in \mathcal{V}$ corresponding to the band $\theta_{0}(x, v, \alpha) \leq f(x, \alpha) \leq \theta_{1}(x, v, \alpha)$. We then propose an extremely

\footnotetext{
3"The support function (of a nonempty closed convex set $B$ in direction $q$ ) $\sigma(q)$ is the signed distance of the support plane to $B$ with exterior normal vector $q$ from the origin; the distance is negative if and only if $q$ points into the open half space containing the origin," Schneider (1993, page 37). See Rockafellar (1970, Chapter 13) or Schneider (1993, Section 1.7) for a thorough discussion of the support function of a closed convex set and its properties.

${ }^{4}$ More recently, Fang and Santos (2018) propose an alternative resampling scheme, which does not require data jittering, and is valid in our context. Their procedure, however, requires the estimation of the directional derivative of the support function.
} 
straightforward, albeit (mildly) conservative, procedure to conduct inference. More powerful inference can be achieved by employing either the precision correction procedure of Chernozhukov, Lee, and Rosen (2013) or the generalized moment selection approach of Andrews and Shi (2013).

Because the support function process is characterized on the entire $\mathcal{S}^{d-1} \times \mathcal{A}$, our functional asymptotic results also allow us to perform inference on statistics that involve a continuum of values for $q$ and/or for $\alpha$. The latter is a substantial advancement compared to the related literature using support function for inference in partially identified models. For example, for best linear approximations to conditional quantile functions with interval outcome data, we are able to test whether a given regressor $x_{j}$ has a positive coefficient in the best linear approximation for all $\alpha \in \mathcal{A}$. When the conditional quantile is in fact linear, rejection of this assumption implies that $x_{j}$ has a non-positive effect for some $\alpha \in \mathcal{A}$.

To illustrate the use of our estimator, we revisit the analysis of Mulligan and Rubinstein (2008) in light of the bounding approach of Blundell, Gosling, Ichimura, and Meghir (2007). The literature studying female labor force participation has argued that the gender wage gap has shrunk between 1975 and 2001. Mulligan and Rubinstein (2008) suggest that women's wages may have grown less than men's wages between 1975 and 2001, had their behavior been held constant, but a selection effect induces the data to show the gender wage gap contracting. They point out that a growing wage inequality within gender induces women to invest more in their market productivity. In turn, this would differentially pull high skilled women into the workplace and the selection effect may make it appear as if cross-gender wage inequality had declined. To test this conjecture they employ a Heckman selection model to correct married women's conditional mean wages for selectivity and investment biases. Using CPS repeated cross-sections from 1975-2001 they argue that the selection of women into the labor market has changed sign, from negative to positive, or at least that positive selectivity bias has come to overwhelm investment bias. Specifically, they find that the gender wage gap measured by OLS decreased from -0.419 in 1975-1979 to -0.256 in 19951999. After correcting for selection using the classic Heckman selection model, they find that the wage gap was -0.379 in 1975-1979 and -0.358 in 1995-1999, thereby concluding that correcting for selection, the gender wage gap may have not shrunk at all. Because it is well known that without a strong exclusion restriction results of the normal selection model can be unreliable, Mulligan and Rubinstein conduct a sensitivity analysis which corroborates their findings.

We provide an alternative approach. We use our method to estimate bounds on the quantile gender wage gap for the 1970s and the 1990s, without assuming a parametric form of selection or a strong exclusion restriction. When selection into the labor force if left completely unrestricted, we are able to document a gender wage gap for quantiles up to the 0.4. We then augment our analysis with additional assumptions, following the identification analysis earlier put forward by Blundell, Gosling, Ichimura, and Meghir (2007) to study the gender wage gap in the UK. Under the assumption that the median wage offer of the employed is larger than that of individuals that do not work, the evidence of a gender wage gap extends to quantiles up to the 0.7. When the assumption is further strengthened to require stochastic dominance, the evidence of a gender wage gap extends to all quantiles, and 
there is some evidence at the 0.8 and higher quantiles that the gender wage gap decreased between the 1970s and 1990s.

Related Literature. This paper contributes to a growing literature on inference on setidentified parameters. Important examples in the literature include, among others, Andrews and Barwick (2012), Andrews and Shi (2013), Andrews and Soares (2010), BM, Bontemps, Magnac, and Maurin (2012), Bugni (2010), Canay (2010), Chernozhukov, Hong, and Tamer (2007), Chernozhukov, Lee, and Rosen (2013), Galichon and Henry (2009), Kaido (2016), Kaido and Santos (2014), Romano and Shaikh (2008), Romano and Shaikh (2010), and Rosen (2008). BM propose an approach for estimation and inference in models where the identification region is equal to the Aumann expectation of a properly defined random set that can be constructed from observable random variables, and can therefore be estimated via Minkowski averages of sample counterparts. Building on the fundamental insight in random set theory that convex compact sets can be represented via their support functions to leverage limit theorems for stochastic processes (Artstein and Vitale (1975)), BM propose a support function based framework for inference in partially identified models. They use best linear prediction as their main illustration. Bontemps, Magnac, and Maurin (2012) extend the results of BM in important directions, by allowing for incomplete linear moment restrictions where the number of restrictions exceeds the number of parameters to be estimated, and extend the familiar Sargan test for overidentifying restrictions to partially identified models. Kaido (2016) establishes a duality between the criterion function approach proposed by Chernozhukov, Hong, and Tamer (2007), and the support function approach proposed by BM. Kaido and Santos (2014) establish that support function based estimators for identified sets resulting from convex moment inequalities are asymptotically efficient, including the estimator proposed by BM.

Closely related to the application of our method to the sample selection example, is the work of Kline and Santos (2013). They study the sensitivity of empirical conclusions about conditional quantile functions to the presence of missing outcome data, when the Kolmogorov-Smirnov distance between the conditional distribution of observed outcomes and the conditional distribution of missing outcomes is bounded by some constant $k$ across all values of the covariates. Under these assumptions, Kline and Santos show that the conditional quantile function is sandwiched between a lower and an upper band, indexed by the level of the quantile and the constant $k$. They conduct inference pointiwise in $\alpha$, under the assumptions that: (i) the support of the covariates is finite, so that the lower and upper bands can be estimated at parametric rates; and (ii) the distribution of $x$ is known, thereby obtaining that $\mathrm{E}\left(x x^{\prime}\right)$ is known. Each of these assumptions significantly simplifies the derivation of the asymptotic distribution, but is often not warranted in applications. The former assumption rules out the presence of any continuous regressor, while the latter introduces a strong element of arbitrariness in the analysis. In sharp contrast, our results are derived without imposing any of these assumptions, hold uniformly in $\alpha$, and allow the researcher to utilize instruments. While technically challenging, allowing for non-parametric estimates of the bounding functions and for intersection bounds considerably expands the domain of applicability of our approach, while allowing for an unknown population distribution on $x$ eliminates a significant element of arbitrariness from the analysis. 
Structure of the Paper. Section 2 develops our framework, and Section 3 demonstrates its versatility by applying it to quantile regression, distribution regression, sample selection problems, and treatment effects. Section 4 provides an overview of our theoretical results and describes the estimation and inference procedures. Section 5 reports results for our empirical application. Section 6 concludes. All proofs are in the Appendix.

\section{The General Framework}

We propose a method to carry out inference for best linear approximations to the set of functions $\mathfrak{F}(\alpha)$ defined in equation (1.1). We let $x$ denote a (column) vector in $\mathbb{R}^{d}$, and $\alpha \in \mathcal{A}$ some index with $\mathcal{A}$ a compact set. For example, in quantile regression $\alpha$ denotes a quantile; in duration regression $\alpha$ denotes a failure time. We assume that for each $x$ the bounding functions $\theta_{0}(x, \alpha)$ and $\theta_{1}(x, \alpha)$ are absolutely integrable. We note that even in the special case where $f(\cdot, \alpha)$ is linear in $x$, the bounding functions $\theta_{0}(\cdot, \alpha), \theta_{1}(\cdot, \alpha)$ need not be linear in $x$ and economic theory may provide little guidance on their functional form. Therefore, our asymptotic results allow for non-parametric estimation of these functions via series methods.

If the true function of interest $f(\cdot, \alpha)$ were point identified, we could approximate it with a linear function by choosing coefficients $\beta(\alpha)$ to minimize the expected squared prediction error $\mathrm{E}\left[\left(f(x, \alpha)-x^{\prime} \beta(\alpha)\right)^{2}\right]$ under the assumption that $\mathrm{E}\left[x x^{\prime}\right]$ is full rank. Because $f(\cdot, \alpha)$ is only known to lie in $\mathfrak{F}(\alpha)$, performing this operation for each admissible function $\phi(\cdot, \alpha) \in$ $\mathfrak{F}(\alpha)$ yields a set of observationally equivalent parameter vectors, denoted $B(\alpha)$ :

$$
\begin{aligned}
B(\alpha) & =\left\{\beta \in \mathbb{R}^{d}: \beta=\underset{b}{\arg \min } \mathrm{E}\left[\left(\phi(x, \alpha)-x^{\prime} b\right)^{2}\right], \mathrm{P}\left(\theta_{0}(x, \alpha) \leq \phi(x, \alpha) \leq \theta_{1}(x, \alpha)\right)=1\right\} \\
& =\left\{\beta \in \mathbb{R}^{d}: \beta=\mathrm{E}\left[x x^{\prime}\right]^{-1} \mathrm{E}[x \phi(x, \alpha)], \mathrm{P}\left(\theta_{0}(x, \alpha) \leq \phi(x, \alpha) \leq \theta_{1}(x, \alpha)\right)=1\right\} .
\end{aligned}
$$

It is easy to see that the set $B(\alpha)$ is almost surely non-empty, compact, and convex valued, because it is obtained by applying linear operators to the (random) almost surely nonempty interval $\left[\theta_{0}(x, \alpha), \theta_{1}(x, \alpha)\right]$, see BM (Section 4) for a discussion. Hence, $B(\alpha)$ can be characterized quite easily through its support function

$$
\sigma(q, \alpha):=\sup _{\beta(\alpha) \in B(\alpha)} q^{\prime} \beta(\alpha)
$$

which takes on almost surely finite values $\forall q \in \mathcal{S}^{d-1}$. In fact,

$$
B(\alpha)=\bigcap_{q \in \mathcal{S}^{d-1}}\left\{b: q^{\prime} b \leq \sigma(q, \alpha)\right\},
$$

see Rockafellar (1970, Chapter 13). The support function provides a convenient way to compute projections of the identified set. These can be used to report upper and lower bounds on individual coefficients and draw two-dimensional identification regions for pairs of coefficients. For example, the bound for the $k$ th component of $\beta(\alpha)$ is $\left[-\sigma\left(-e_{k}, \alpha\right), \sigma\left(e_{k}, \alpha\right)\right]$, where $e_{k}$ is the $k$ th standard basis vector. Similarly, the bound for a linear combination of the coefficients, $q^{\prime} \beta(\alpha)$, is $[-\sigma(-q, \alpha), \sigma(q, \alpha)]$. Figure 2.1 provides an illustration. In this example, $\beta$ is three dimensional. The left panel shows the entire identified set. The right panel shows the joint identification region for $\beta_{1}$ and $\beta_{2}$. The identified intervals for $\beta_{1}$ and $\beta_{2}$ are also marked in red on the right panel. 
FiguRE 2.1. Identification region and its projections
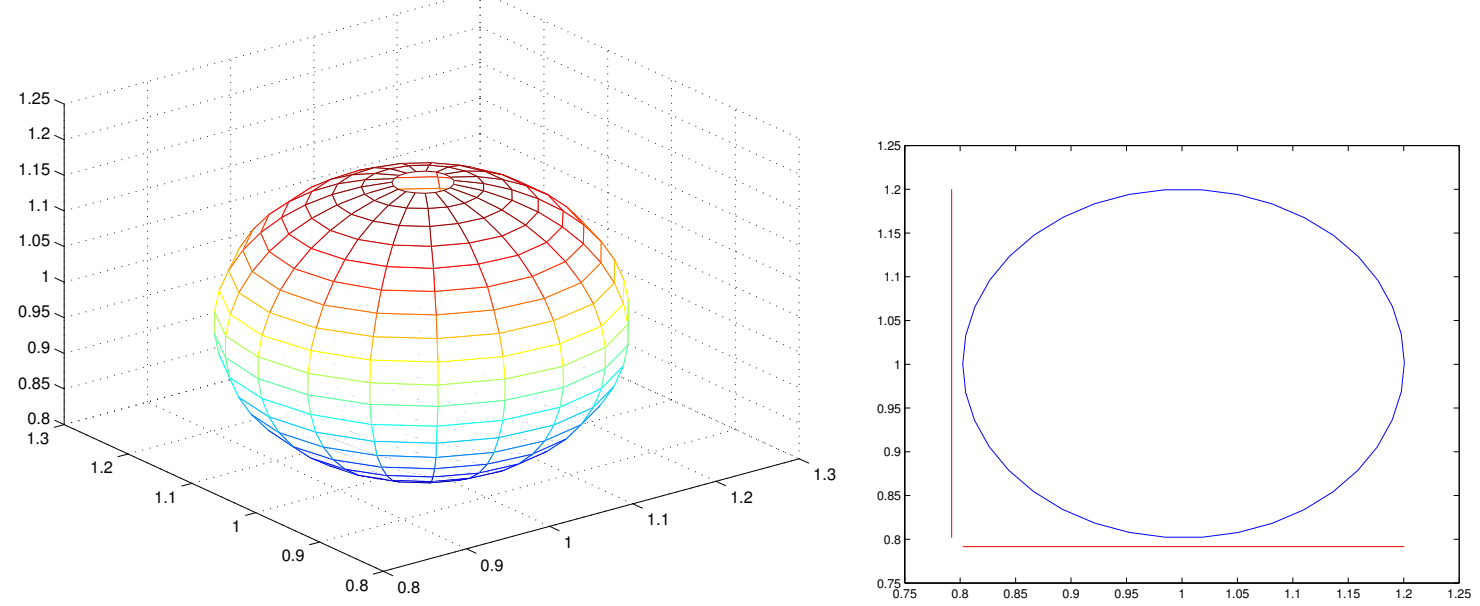

More generally, if the criterion for "best" linear approximation is to minimize $\mathrm{E}[(f(x, \alpha)-$ $\left.\left.x^{\prime} b(\alpha)\right) \tilde{z}^{\prime} W \tilde{z}\left(f(x, \alpha)-x^{\prime} b(\alpha)\right)\right]$, where $W$ is a $j \times j$ weight matrix and $\tilde{z}$ a $j \times 1$ vector of instruments, then we have

$B(\alpha)=\left\{\beta \in \mathbb{R}^{d}: \beta=\mathrm{E}\left[x \tilde{z}^{\prime} W \tilde{z} x^{\prime}\right]^{-1} \mathrm{E}\left[x \tilde{z}^{\prime} W \tilde{z} \phi(x, \alpha)\right], \mathrm{P}\left(\theta_{0}(x, \alpha) \leq \phi(x, \alpha) \leq \theta_{1}(x, \alpha)\right)=1\right\}$.

As in Bontemps, Magnac, and Maurin (2012), Magnac and Maurin (2008), and BM (p. 807 ) the support function of $B(\alpha)$ can be shown to be ${ }^{5}$

$$
\sigma(q, \alpha)=\mathrm{E}\left[z_{q} w_{q}\right]
$$

where

$$
\begin{aligned}
z & =x \tilde{z}^{\prime} W \tilde{z}, z_{q}=q^{\prime} \mathrm{E}\left[x z^{\prime}\right]^{-1} z, \\
w_{q} & =\theta_{1}(x, \alpha) 1\left(z_{q}>0\right)+\theta_{0}(x, \alpha) 1\left(z_{q} \leq 0\right) .
\end{aligned}
$$

We estimate the support function by plugging in estimates of $\theta_{\ell}(x, \alpha), \ell=0,1$, and taking empirical expectations:

$$
\widehat{\sigma}(q, \alpha)=\mathbb{E}_{n}\left[q^{\prime}\left(\mathbb{E}_{n}\left[x_{i} z_{i}^{\prime}\right]\right)^{-1} z_{i}\left(\widehat{\theta}_{1}\left(x_{i}, \alpha\right) 1\left(\widehat{z}_{i q}>0\right)+\widehat{\theta}_{0}\left(x_{i}, \alpha\right) 1\left(\widehat{z}_{i q} \leq 0\right)\right)\right],
$$

where $\mathbb{E}_{n}$ denotes the empirical expectation, $\widehat{z}_{i q}=q^{\prime}\left(\mathbb{E}_{n}\left[x_{i} z_{i}^{\prime}\right]\right)^{-1} z_{i}$, and $\widehat{\theta}_{\ell}(x, \alpha), \ell=0,1$, are the estimators of $\theta_{\ell}(x, \alpha)$.

\footnotetext{
${ }^{5}$ To further illustrate, suppose that $z=x=\left[1 ; x_{1}\right]$, with $x_{1}$ a scalar random variable, so $\beta(\alpha)=$ $\left[\beta_{0}(\alpha) \quad \beta_{1}(\alpha)\right]$. In most applications, $\beta_{1}(\alpha)$ is the primary object of interest. Setting $q=[0 \pm 1]$, BM and Bontemps, Magnac, and Maurin (2012) give explicit formulae for the upper and lower bound of $\beta_{1}(\alpha)$ :

$$
\begin{aligned}
& \underline{\beta}_{1}(\alpha)=\inf _{f_{i} \in\left[\theta_{i 0}, \theta_{i 1}\right]} \frac{\operatorname{cov}\left(x_{1 i}, f_{i}\right)}{\operatorname{var}\left(x_{i 1}\right)}=\frac{\mathrm{E}\left[\left(x_{1 i}-\mathrm{E}\left[x_{1 i}\right]\right)\left(\theta_{1 i} 1\left\{x_{1 i}<\mathrm{E}\left[x_{1 i}\right]\right\}+\theta_{0 i} 1\left\{x_{1 i}>\mathrm{E}\left[x_{1 i}\right]\right\}\right)\right]}{\mathrm{E}\left[x_{1 i}^{2}\right]-\mathrm{E}\left[x_{1 i}\right]^{2}} \\
& \bar{\beta}_{1}(\alpha)=\sup _{f_{i} \in\left[\theta_{i 0}, \theta_{i 1}\right]} \frac{\operatorname{cov}\left(x_{1 i}, f_{i}\right)}{\operatorname{var}\left(x_{i 1}\right)}=\frac{\mathrm{E}\left[\left(x_{1 i}-\mathrm{E}\left[x_{1 i}\right]\right)\left(\theta_{1 i} 1\left\{x_{1 i}>\mathrm{E}\left[x_{1 i}\right]\right\}+\theta_{0 i} 1\left\{x_{1 i}<\mathrm{E}\left[x_{1 i}\right]\right\}\right)\right]}{\mathrm{E}\left[x_{1 i}^{2}\right]-\mathrm{E}\left[x_{1 i}\right]^{2}}
\end{aligned}
$$

where $\theta_{0 i}=\theta_{0}\left(x_{i}, \alpha\right)$ and $\theta_{1 i}=\theta_{1}\left(x_{i}, \alpha\right)$, see also Stoye $(2007)$.
} 


\section{Motivating Examples}

3.1. Interval valued data. Analysis of regression with interval valued data has become a canonical example in the partial identification literature due to the widespread presence of such data. The Health and Retirement Study is one of the first instances where income data is collected from respondents in the form of brackets, with degenerate (singleton) intervals for individuals who opt to fully reveal their income (see, e.g. Juster and Suzman (1995)). The Occupational Employment Statistics (OES) program at the Bureau of Labor Statistics collects wage data from employers as intervals, and uses these data to construct estimates for wage and salary workers in 22 major occupational groups and 801 detailed occupations. Due to concerns for privacy, public use tax data are recorded as the number of tax payers which belong to each of a finite number of cells, see e.g. Picketty (2005) ${ }^{6}$

3.1.1. Interval valued y. BM and Bontemps, Magnac, and Maurin (2012), among others, have focused on estimation of best linear approximations to conditional expectation functions with interval outcome data. Our framework covers the conditional expectation case, as well as an extension to quantile regression wherein we set identify $\beta(\alpha)$ across all quantiles $\alpha \in \mathcal{A}$. To avoid redundancy with the related literature, here we describe the setup for quantile regression. Let the $\alpha$-th conditional quantile of $y \mid x$ be denoted $Q_{y}(\alpha \mid x)$. We are interested in a linear approximation $x^{\prime} \beta(\alpha)$ to this function. However, we do not observe $y$. Instead we observe $y_{0}$ and $y_{1}$, with $\mathrm{P}\left(y_{0} \leq y \leq y_{1}\right)=1$. It is immediate that

$$
Q_{y_{0}}(\alpha \mid x) \leq Q_{y}(\alpha \mid x) \leq Q_{y_{1}}(\alpha \mid x) \quad x-\text { a.s. }
$$

where $Q_{y_{\ell}}(\alpha \mid x)$ is the $\alpha$-th conditional quantile of $y_{\ell} \mid x, \ell=0,1$. Hence, the identification region $B(\alpha)$ is as in equation $(2.1)$, with $\theta_{\ell}(x, \alpha)=Q_{y_{\ell}}(\alpha \mid x)$.

3.1.2. Interval valued $x$. Consider now inference on functionals of $\mathrm{P}(y \mid x)$, when $y$ is perfectly observed but $x$ is only learned to lie in the interval $\left[x_{0}, x_{1}\right]$, as $\mathrm{P}\left(x_{0} \leq x \leq x_{1}\right)=1$, with $x_{0}, x_{1}$ observed. Our methodology applies to the framework of Manski and Tamer (2002), extended to the case of quantile regression $7^{7}$ Following Manski and Tamer, we assume that the conditional expectation (respectively, quantile) of $y \mid x$ is weakly monotonic in $x$, say nondecreasing, and mean independent of $x_{0}, x_{1}$ conditional on $x$ (respectively, $\left.Q_{y}\left(\alpha \mid x, x_{0}, x_{1}\right)=Q_{y}(\alpha \mid x)\right)$. Manski and Tamer show that

$$
\sup _{x_{1} \leq x} \mathrm{E}\left(y \mid x_{0}, x_{1}\right) \leq \mathrm{E}(y \mid x) \leq \inf _{x_{0} \geq x} \mathrm{E}\left(y \mid x_{0}, x_{1}\right),
$$

and similar reasoning yields

$$
\sup _{x_{1} \leq x} Q_{y}\left(\alpha \mid x_{0}, x_{1}\right) \leq Q_{y}(\alpha \mid x) \leq \inf _{x_{0} \geq x} Q_{y}\left(\alpha \mid x_{0}, x_{1}\right) .
$$

\footnotetext{
${ }^{6}$ See Manski and Tamer (2002) and Bontemps, Magnac, and Maurin (2012) for more examples.

${ }^{7}$ Our approach also applies to the framework of Magnac and Maurin (2008), who study identification in semi-parametric binary regression models with regressors that are either discrete or measured by intervals, under an uncorrelated error assumption, a conditional independence assumption between error and interval/discrete valued regressor, and a finite support assumption.
} 
Hence, the identification region $B(\alpha)$ corresponding to (3.1) is as in equation (2.1), with $\theta_{0}(x, \alpha)=\sup _{x_{1} \leq x} \mathrm{E}\left(y \mid x_{0}, x_{1}\right)$ and $\theta_{1}(x, \alpha)=\inf _{x_{0} \geq x} \mathrm{E}\left(y \mid x_{0}, x_{1}\right)$, while the the identification region $B(\alpha)$ corresponding to $(3.2)$ is as in equation (2.1), with $\theta_{0}(x, \alpha)=\sup _{x_{1} \leq x} Q_{y}\left(\alpha \mid x_{0}, x_{1}\right)$ and $\theta_{1}(x, \alpha)=\inf _{x_{0} \geq x} Q_{y}\left(\alpha \mid x_{0}, x_{1}\right)$.

3.2. Distribution and duration regression with interval outcome data. Distribution regression with interval valued data is another application of our method. We consider models in which the conditional distribution of $y \mid x$ is given by

$$
\mathrm{P}(y \leq \alpha \mid x) \equiv F_{y \mid x}(\alpha \mid x)=\Phi(f(x, \alpha))
$$

where $\Phi($.$) is a known one-to-one link function. A special case of this class of models is$ the duration model, wherein we have $f(\alpha, x)=g(\alpha)+\gamma(x)$, where $g($.$) is a monotonic$ function. As in the quantile regression example, assume that we observe $\left(y_{0}, y_{1}, x\right)$ with $\mathrm{P}\left(y_{0} \leq y \leq y_{1}\right)=1$. Then

$$
\Phi^{-1}\left(F_{y_{1} \mid x}(\alpha \mid x)\right) \leq f(x, \alpha) \leq \Phi^{-1}\left(F_{y_{0} \mid x}(\alpha \mid x)\right) .
$$

Hence, $B(\alpha)$ is as in equation (2.1), with $\theta_{\ell}(x, \alpha)=F_{y_{1-\ell} \mid x}(\alpha \mid x), \ell=0,1$ and the operator $\Phi^{-1}$ applied to the bounding functions. A leading example, following Han and Hausman (1990) and Foresi and Peracchi (1995), takes this form with $\Phi$ a probit or logit link function.

3.3. Sample Selection. Sample selection is a commonplace problem in the empirical analysis of important economic phenomena, including labor force participation, skill composition of immigrants, returns to education, program evaluation, productivity estimation, insurance, models with occupational choice and financial intermediation (for recent examples, see respectively Mulligan and Rubinstein (2008), Jasso and Rosenzweig (2008), Card (1999), Imbens and Wooldridge (2009), Olley and Pakes (1996), Einav, Finkelstein, Ryan, Schrimpf, and Cullen (2013), Townsend and Urzua (2009)). In Section 5 we use our methodology in conjunction with the bounding approach of Blundell, Gosling, Ichimura, and Meghir (2007) to revisit the analysis of Mulligan and Rubinstein (2008), who confront selection in the context of female labor supply.

Consider a standard sample selection model. We are interested in the behavior of $y$ conditional on $x$; however, we only observe $y$ when $u=1$. Manski (1994) shows that the sharp bounds on the conditional quantile function of $y$ are

$$
\begin{aligned}
Q_{0}(\alpha \mid x) & = \begin{cases}Q_{y}\left(\frac{\alpha-\mathrm{P}(u=0 \mid x)}{\mathrm{P}(u=1 \mid x)} \mid x, u=1\right) & \text { if } \alpha \geq \mathrm{P}(u=0 \mid x) \\
y_{0} & \text { otherwise }\end{cases} \\
Q_{1}(\alpha \mid x) & = \begin{cases}Q_{y}\left(\frac{\alpha}{\mathrm{P}(u=1 \mid x)} \mid x, u=1\right) & \text { if } \alpha \leq \mathrm{P}(u=1 \mid x) \\
y_{1} & \text { otherwise }\end{cases}
\end{aligned}
$$

where $y_{0}$ is the smallest possible value that $y$ can take (possibly $-\infty$ ) and $y_{1}$ is the largest possible value that $y$ can take (possibly $+\infty$ ). Thus, we obtain

$$
Q_{0}(\alpha \mid x) \leq Q_{y}(\alpha \mid x) \leq Q_{1}(\alpha \mid x) .
$$

and the corresponding set of coefficients of linear approximations to $Q_{y}(\alpha \mid x)$ is as in equation (2.1), with $\theta_{\ell}(x, \alpha)=Q_{\ell}(\alpha \mid x), \ell=0,1$. 
3.3.1. Alternative Form for the Bounds. As written above, the expressions for $Q_{0}(\alpha \mid x)$ and $Q_{1}(\alpha \mid x)$ involve the propensity score, $\mathrm{P}(u \mid x)$ and several different conditional quantiles of $y \mid u=1$. Estimating these objects might be computationally intensive. We provide a simplification by working with

$$
\tilde{y}_{0}=y 1\{u=1\}+y_{0} 1\{u=0\}, \quad \tilde{y}_{1}=y 1\{u=1\}+y_{1} 1\{u=0\} .
$$

One can easily verify that $Q_{\tilde{y}_{\ell}}(\alpha \mid x)=Q_{\ell}(\alpha \mid x), \ell=0,1$, hence the bounds on the conditional quantile function can be obtained without calculating the propensity score.

3.3.2. Sample Selection with an Exclusion Restriction. Often when facing selection problems researchers impose exclusion restrictions. That is, they assume that there are some components of $x$ that affect $\mathrm{P}(u=1 \mid x)$, but not $F(y \mid x)$. Availability of such an instrument, denoted $v$, can help shrink the bounds on $Q_{y}(\alpha \mid x)$. For concreteness, we replace $x$ with $(x, v)$ we assume that $F(y \mid x, v)=F(y \mid x) \forall v \in \mathcal{V}_{x}$, with $\mathcal{V}_{x}$ denoting the support of $v$ given $x$, and we assume that $\mathcal{V}_{x}$ is finite $x-$ a.s. To simplify notation, we assume $\mathcal{V}_{x}=\mathcal{V}$ for all $x$, but we note that our approach is valid in the absence of this restriction. Using again Manski (1994), for each $v \in \mathcal{V}$ the bounds on the conditional quantile function are:

$$
Q_{0}(\alpha \mid x, v) \leq Q_{y}(\alpha \mid x) \leq Q_{1}(\alpha \mid x, v) \forall v \in \mathcal{V},
$$

and therefore

$$
\sup _{v \in \mathcal{V}} Q_{0}(\alpha \mid x, v) \leq Q_{y}(\alpha \mid x) \leq \inf _{v \in \mathcal{V}} Q_{1}(\alpha \mid x, v)
$$

where $Q_{\ell}(\alpha \mid x, v), \ell=0,1$, are defined similarly to the previous section with $x$ replaced by $(x, v)$, and as before we can avoid computing the propensity score by constructing the variables $\tilde{y}_{\ell}, \ell=0,1$ as in equation (3.3). Then $Q_{\tilde{y}_{\ell}}(\alpha \mid x, v)=Q_{\ell}(\alpha \mid x, v)$, and the set of coefficients of linear approximations to $Q_{y}(\alpha \mid x)$ is as in equation $(2.1)$, with $\theta_{0}(x, \alpha)=$ $\sup _{v \in \mathcal{V}} Q_{\tilde{y}_{0}}(\alpha \mid x, v)$ and $\theta_{1}(x, \alpha)=\inf _{v \in \mathcal{V}} Q_{\tilde{y}_{1}}(\alpha \mid x, v)$.

3.4. Average, Quantile, and Distribution Treatment Effects. Researchers are often interested in mean, quantile, and distributional treatment effects. Our framework easily accommodates these examples. Let $y_{i}^{C}$ denote the outcome for person $i$ if she does not receive treatment, and $y_{i}^{T}$ denote the outcome for person $i$ if she receives treatment. The methods discussed in the preceding section yield bounds on the conditional quantiles of these outcomes. In turn, these bounds can be used to obtain bounds on the quantile treatment effect as follows:

$\sup _{v \in \mathcal{V}} Q_{0}^{T}(\alpha \mid x, v)-\inf _{v \in \mathcal{V}} Q_{1}^{C}(\alpha \mid x, v) \leq Q_{y^{T}}(\alpha \mid x)-Q_{y^{C}}(\alpha \mid x) \leq \inf _{v \in \mathcal{V}} Q_{1}^{T}(\alpha \mid x, v)-\sup _{v \in \mathcal{V}_{x}} Q_{0}^{C}(\alpha \mid x, v)$,

and again the corresponding set of coefficients of linear approximations to $Q_{y^{T}}(\alpha \mid x)-$ $Q_{y^{C}}(\alpha \mid x)$ is as in equation 2.1, with $\theta_{0}(x, \alpha)=\sup _{v \in \mathcal{V}} Q_{0}^{T}(\alpha \mid x, v)-\inf _{v \in \mathcal{V}} Q_{1}^{C}(\alpha \mid x, v)$, and $\theta_{1}(x, \alpha)=\inf _{v \in \mathcal{V}} Q_{1}^{T}(\alpha \mid x, v)-\sup _{v \in \mathcal{V}_{x}} Q_{0}^{C}(\alpha \mid x, v)$.

Analogous bounds apply for the distribution treatment effect and the mean treatment effect 8

\footnotetext{
${ }^{8}$ Interval regressors can also be accommodated, by adapting the results in Section 3.1.
} 


\section{Estimation And Inference}

4.1. Overview of the Results. This section provides an overview of our results, and explains how these can be applied in practice. As described in section 2, our goal is to estimate the support function, $\sigma(q, \alpha)$.

4.1.1. Use of asymptotic results. We develop limit theory that allows us to (1) derive the asymptotic distribution of the support function process; (2) provide inferential procedures; and (3) establish validity of the Bayesian bootstrap. Bootstrapping is especially important for practitioners, because of the potential complexity of the covariance functions involved in the limiting distributions.

Limit theory and bootstrap. We show that the support function process $S_{n}(t):=\sqrt{n}\left(\widehat{\sigma}(t)-\sigma_{0}(t)\right)$, with $t \in T:=\mathcal{S}^{d-1} \times \mathcal{A}$, is strongly approximated by a Gaussian process on $T$ :

$$
S_{n}(t)=\mathbb{G}\left[h_{k}(t)\right]+o_{\mathrm{P}}(1)
$$

in $\ell^{\infty}(T)$, where $\ell^{\infty}(T)$ denotes the set of all uniformly bounded real functions on $T, k$ denotes the number of series terms in our non-parametric estimator of $\theta_{\ell}(x, \alpha), \ell=0,1$, and $h_{k}(t)$ denotes a stochastic process carefully defined in Section 4.3 . Here, $\mathbb{G}\left[h_{k}(t)\right]$ is a tight P-Brownian bridge with covariance function $\Omega_{k}\left(t, t^{\prime}\right)=\mathrm{E}\left[h_{k}(t) h_{k}\left(t^{\prime}\right)\right]-\mathrm{E}\left[h_{k}(t)\right] \mathrm{E}\left[h_{k}\left(t^{\prime}\right)\right]$. We show that while the sequence of processes $\mathbb{G}\left[h_{k}(t)\right]$ may not necessarily converge weakly when $k \rightarrow \infty$, each subsequence has a further subsequence converging to a tight Gaussian process in $\ell^{\infty}(T)$ with a non-degenerate covariance function.

We show how to conduct inference using the quantiles of the sequence $\mathbb{G}\left[h_{k}(t)\right]$. Specifically, if we have a continuous function $f$ that satisfies certain (non-restrictive) conditions detailed in Section 4.3.9 and $\widehat{c}_{n}(1-\tau)=c_{n}(1-\tau)+o_{\mathrm{P}}(1)$ is a consistent estimator of the $(1-\tau)$-quantile of $f\left(\mathbb{G}\left[h_{k}(t)\right]\right)$, given by $c_{n}(1-\tau)$, then

$$
\mathrm{P}\left\{f\left(S_{n}\right) \leq \widehat{c}_{n}(1-\tau)\right\} \rightarrow 1-\tau .
$$

Finally, we consider the limiting distribution of the Bayesian bootstrap version of the support function process, denoted $\widetilde{S}_{n}(t):=\sqrt{n}(\tilde{\sigma}(t)-\widehat{\sigma}(t))$, and show that, conditional on the data, it admits an approximation

$$
\left.\tilde{S}_{n}(t)=\widetilde{\mathbb{G}} \widetilde{\left[h_{k}(t)\right.}\right]+o_{\mathrm{Pe}}(1)
$$

where $\left.\widetilde{\mathbb{G}} \widetilde{h_{k}(t)}\right]$ has the same distribution as $\mathbb{G}\left[h_{k}(t)\right]$ and is independent of $\mathbb{G}\left[h_{k}(t)\right]$, and $\mathrm{P}^{\mathrm{e}}$ denotes the probability measure conditional on the data. Since the bootstrap distribution is asymptotically close to the true distribution of interest, this allows us to perform many standard and some less standard inferential tasks.

Pointwise asymptotics. Suppose we want to form a confidence interval for $q^{\prime} \beta(\alpha)$ for some fixed $q$ and $\alpha$. Since our estimator can be approximated by a sequence of Gaussian processes, we know that

$$
\sqrt{n}\left(\begin{array}{c}
-\widehat{\sigma}(-q, \alpha)+\sigma_{0}(-q, \alpha) \\
\widehat{\sigma}(q, \alpha)-\sigma_{0}(q, \alpha)
\end{array}\right) \approx_{d} N\left(0, \Omega_{k}(q, \alpha)\right)
$$

\footnotetext{
${ }^{9}$ For example, functions yielding test statistics based on the directed Hausdorff distance and on the Hausdorff distance (see, e.g., BM) satisfy these conditions.
} 
To form a confidence interval that covers the bound on $q^{\prime} \beta(\alpha)$ with probability $1-\tau$ we can take

$$
-\widehat{\sigma}(-q, \alpha)+n^{1 / 2} \widehat{C}_{\tau / 2}(q, \alpha) \leq q^{\prime} \beta(\alpha) \leq \widehat{\sigma}(q, \alpha)+n^{-1 / 2} \widehat{C}_{1-\tau / 2}(q, \alpha)
$$

where the critical values, $\widehat{C}_{\tau / 2}(q, \alpha)$ and $\widehat{C}_{1-\tau / 2}(q, \alpha)$, are such that if $\left(x_{1} \quad x_{2}\right)^{\prime} \sim N\left(0, \Omega_{k}(q, \alpha)\right)$, then

$$
\mathrm{P}\left(x_{1} \geq \widehat{C}_{\tau / 2}(q, \alpha), x_{2} \leq \widehat{C}_{1-\tau / 2}(q, \alpha)\right)=1-\tau+o_{p}(1)
$$

If we had a consistent estimate of $\Omega_{k}(q, \alpha)$, we would be able to set

$$
\left(\begin{array}{c}
\widehat{C}_{\tau / 2}(q, \alpha) \\
\widehat{C}_{1-\tau / 2}(q, \alpha)
\end{array}\right)=\widehat{\Omega}_{k}^{1 / 2}(q, \alpha)\left(\begin{array}{c}
-\Phi^{-1}(\sqrt{1-\tau}) \\
\Phi^{-1}(\sqrt{1-\tau})
\end{array}\right)
$$

where $\Phi^{-1}(\cdot)$ is the inverse normal distribution function. However, the formula for $\Omega_{k}(q, \alpha)$ is complicated and it can be difficult to estimate. Therefore, we recommend using a Bayesian bootstrap procedure to estimate the critical values and we provide theoretical justification for it. See section 4.1 .3 for details 10

Functional asymptotics. Because our asymptotic results yield a functional approximation by Gaussian processes of $S_{n}(q, \alpha)$ on the entire $\mathcal{S}^{d-1} \times \mathcal{A}$, we can also perform inference on statistics that involve a continuum of values of $q$ and/or $\alpha$. For example, in our application to quantile regression with selectively observed data, we might be interested in whether covariate $j$ has an effect on the outcome distribution. When translated to our best linear approximation setting, the hypothesis becomes

$$
H_{0}: 0 \in\left[-\sigma_{0}(-q, \alpha), \sigma_{0}(q, \alpha)\right] \forall \alpha \in \mathcal{A},
$$

with $q=e_{j}$. When the conditional quantile is in fact linear, rejection of this assumption implies that $x_{j}$ has a non-zero effect for some $\alpha \in \mathcal{A}$. A natural family of test statistics is

$$
\mathcal{T}_{n}=\sqrt{n} \sup _{\alpha \in \mathcal{A}}(1\{-\widehat{\sigma}(-q, \alpha)>0\}|\widehat{\sigma}(-q, \alpha)| \rho(-q, \alpha) \vee 1\{\widehat{\sigma}(q, \alpha)<0\}|\widehat{\sigma}(q, \alpha)| \rho(q, \alpha))
$$

where $\rho(q, \alpha) \geq 0$ is some weighting function which can be chosen to maximize weighted power against some family of alternatives. There are many values of $\sigma_{0}(q, \alpha)$ consistent with the null hypothesis, but the one for which it will be hardest to control size is $-\sigma_{0}(-q, \cdot)=\sigma_{0}(q, \cdot)=0$. In this case, we know that $S_{n}(t)=\sqrt{n} \widehat{\sigma}(t), t=(q, \alpha) \in T$, is well approximated by the Gaussian process $\mathbb{G}\left[h_{k}(t)\right]$. Moreover, the quantiles of any functional of $S_{n}(t)$ converge to the quantiles of the same functional applied to $\mathbb{G}\left[h_{k}(t)\right]$. Thus, we could calculate a $\tau$ critical value for $\mathcal{T}_{n}$ by repeatedly simulating a realization of $\mathbb{G}\left[h_{k}(q, \cdot)\right]$, computing $\mathcal{T}_{n}\left(\mathbb{G}\left[h_{k}(q, \cdot)\right]\right)$, and then taking the $(1-\tau)$-quantile of the simulated values of $\mathcal{T}_{n}\left(\mathbb{G}\left[h_{k}(q, \cdot)\right]\right)$. Simulating $\mathbb{G}\left[h_{k}(t)\right]$, however, requires estimating the covariance function. As stated above, the formula for this function is complicated and it can be difficult to estimate. Therefore, we recommend using the Bayesian bootstrap to compute the critical values. Theorem 4 proves that this bootstrap procedure yields consistent inference. Section 4.1 .3 gives a more detailed outline of how to implement this bootstrap. Similar reasoning

\footnotetext{
${ }^{10}$ Instead, if one believes there is some true value $q^{\prime} \beta_{0}(\alpha)$ in the identified set, and one wants to cover this true value (uniformly) with asymptotic probability $1-\tau$, then one can apply the procedures of Imbens and Manski (2004) and Stoye (2009), as adapted by Bontemps, Magnac, and Maurin (2012).
} 
can be used to test hypotheses involving a set of values of $q$ and construct confidence sets that are uniform in $q$ and/or $\alpha$.

4.1.2. Estimation. The first step in estimating the support function is to estimate $\theta_{0}(x, \alpha)$ and $\theta_{1}(x, \alpha)$. Since economic theory often provides even less guidance about the functional form of these bounding functions than it might about the function of interest, our asymptotic results are written to accommodate non-parametric estimates of $\theta_{0}(x, \alpha)$ and $\theta_{1}(x, \alpha)$. In particular, we allow for series estimators of these functions. In this section we briefly review this approach. Parametric estimation follows as a special case where the number of series terms is fixed. Note that while the method of series estimation described here satisfies the conditions of theorems 1 and 2 below, there might be other suitable methods of estimation for the bounding functions.

In each of the examples in section 3 , except for the case of intersection bounds (e.g. sample selection with an exclusion restriction), series estimates of the bounding functions can be formed as follows. Suppose there is a known function of the data for observation $i$, denoted $y_{i \ell}$, and a known function $m(y, \theta(x, \alpha), \alpha)$ such that

$$
\theta_{\ell}(\cdot, \alpha)=\underset{\theta \in \mathcal{L}^{2}(X, \mathrm{P})}{\arg \min } \mathrm{E}\left[m\left(y_{i \ell}, \theta\left(x_{i}, \alpha\right), \alpha\right)\right],
$$

where $X$ denotes the support of $x$ and $\mathcal{L}^{2}(X, \mathrm{P})$ denotes the space of real-valued functions $g$ such that $\int_{X}|g(x)|^{2} d \mathrm{P}(x)<\infty$. Then we can form an estimate of the function $\theta_{\ell}(\cdot, \alpha)$ by replacing it with its series expansion and taking the empirical expectation in the equation above. That is, obtaining the coefficients

$$
\widehat{\vartheta}_{k, \ell}(\alpha)=\underset{\vartheta}{\arg \min } \mathbb{E}_{n}\left[m\left(y_{i \ell}, p_{k}\left(x_{i}\right)^{\prime} \vartheta, \alpha\right)\right],
$$

and setting

$$
\widehat{\theta}_{l}\left(x_{i}, \alpha\right)=p_{k}\left(x_{i}\right)^{\prime} \widehat{\vartheta}_{k, \ell}(\alpha)
$$

Here, $p_{k}\left(x_{i}\right)$ is a $k \times 1$ vector of series functions evaluated at $x_{i}$. These could be any set of functions that span the space in which $\theta_{\ell}(x, \alpha)$ is contained. Typical examples include polynomials, splines, and trigonometric functions, see Chen (2007). Both the properties of $m(\cdot)$ and the choice of approximating functions affect the rate at which $k$ can grow. We discuss this issue in more detail after stating our regularity conditions in section 4.2 .

With intersection bounds, for concreteness discussed here in the case of sample selection with an exclusion restriction, one can proceed as follows. First, estimate $Q_{\tilde{y}_{\ell}}(\alpha \mid x, v)$, $\ell=0,1$, using the method described above. Next, set $\widehat{\theta}_{1}\left(x_{i}, \alpha\right)=\min _{v \in \mathcal{V}} Q_{\tilde{y}_{1}}(\alpha \mid x, v)$ and $\widehat{\theta}_{0}\left(x_{i}, \alpha\right)=\max _{v \in \mathcal{V}} Q_{\tilde{y}_{0}}(\alpha \mid x, v)$, and proceed as in the previous case. We show below, however, that the set of best linear approximations to the band $\left\{\phi(\cdot, \alpha): \max _{v \in \mathcal{V}} Q_{\tilde{y}_{0}}(\alpha \mid x, v) \leq\right.$ $\left.\phi(x, \alpha) \leq \min _{v \in \mathcal{V}} Q_{\tilde{y}_{1}}(\alpha \mid x, v) x-a . s.\right\}$, is equal to the intersection over $v \in \mathcal{V}$ of the sets of best linear approximations to the bands $\left\{\phi(\cdot, \alpha): Q_{\tilde{y}_{0}}(\alpha \mid x, v) \leq \phi(x, \alpha) \leq Q_{\tilde{y}_{1}}(\alpha \mid x, v) x-\right.$ a.s.\}. Establishing this equivalence allows us to provide a valid and extremely straightforward, albeit (mildly) conservative, procedure for inference also in this case.

4.1.3. Bayesian Bootstrap. We suggest using the Bayesian Bootstrap to conduct inference. In particular, we propose the following algorithm. 
Procedure for Bayesian Bootstrap Estimation of Critical Values.

(1) Simulate each bootstrap draw of $\tilde{\sigma}(q, \alpha)$ :

(a) Draw $e_{i} \sim \exp (1), i=1, \ldots, n, \bar{e}=\mathbb{E}_{n}\left[e_{i}\right]$

(b) Estimate:

$$
\begin{aligned}
\tilde{\vartheta}_{k, \ell}(\alpha) & =\underset{\vartheta}{\arg \min } \mathbb{E}_{n}\left[\frac{e_{i}}{\bar{e}} m\left(y_{i \ell}, p_{k}\left(x_{i}\right)^{\prime} \vartheta, \alpha\right)\right], \\
\tilde{\theta}_{\ell}(x, \alpha) & =p_{k}(x)^{\prime} \tilde{\vartheta}_{k, \ell}(\alpha), \\
\tilde{\Sigma} & =\mathbb{E}_{n}\left[\frac{e_{i}}{\bar{e}} x_{i} z_{i}^{\prime}\right]^{-1}, \\
\tilde{w}_{i, q^{\prime}} \tilde{\Sigma} & =\tilde{\theta}_{1}(x, \alpha) 1\left(q^{\prime} \tilde{\Sigma} z>0\right)+\tilde{\theta}_{0}(x, \alpha) 1\left(q^{\prime} \tilde{\Sigma} z \leq 0\right), \\
\tilde{\sigma}(q, \alpha) & =\mathbb{E}_{n}\left[\frac{e_{i}}{\bar{e}} q^{\prime} \tilde{\Sigma} z_{i} \tilde{w}_{i, q^{\prime}} \tilde{\Sigma}\right] .
\end{aligned}
$$

(2) Denote the bootstrap draws as $\tilde{\sigma}^{(b)}, b=1, \ldots, B$, and let $\tilde{S}_{n}^{(b)}=\sqrt{n}\left(\tilde{\sigma}^{(b)}-\widehat{\sigma}\right)$. To estimate the $1-\tau$ quantile of $f\left(S_{n}\right)$, where $f$ is a continuous function determining the test statistic of interest as detailed in Section 4.3, use the empirical $1-\tau$ quantile of the sample $f\left(\tilde{S}_{n}^{(b)}\right), b=1, \ldots, B$

(3) Confidence intervals for linear combinations of coefficients can be obtained as outlined in Section 4.1.1. Inference on statistics that involve a continuum of values of $q$ and/or $\alpha$ can be obtained as outlined in Section 4.1.1.

4.2. Regularity Conditions. In what follows, we state the assumptions that we maintain to obtain our main results. We then discuss these conditions, and verify them for the examples in Section 3 .

C1 (Smoothness of Covariate Distribution). The covariates $z_{i}$ have a sufficiently smooth distribution, namely for some $0<m \leq 1$, we have that $\mathrm{P}\left(\left|q^{\prime} \Sigma z_{i} /\left\|z_{i}\right\|\right|<\delta\right) / \delta^{m} \lesssim 1$ as $\delta \searrow 0$ uniformly in $q \in \mathcal{S}^{d-1}$, with $d$ the dimension of $x$. The matrix $\Sigma=\left(\mathrm{E}\left[x_{i} z_{i}^{\prime}\right]\right)^{-1}$ is finite and invertible.

C2 (Linearization for the Estimator of Bounding Functions ). Let $\bar{\theta}$ denote either the unweighted estimator $\widehat{\theta}$ or the weighted estimator $\tilde{\theta}$, and let $v_{i}=1$ for the case of the unweighted estimator, and $v_{i}=e_{i}$ for the case of the weighted estimator. We assume that for each $\ell=0,1$ the estimator $\bar{\theta}_{\ell}$ admits a linearization of the form:

$$
\sqrt{n}\left(\bar{\theta}_{\ell}(x, \alpha)-\theta_{\ell}(x, \alpha)\right)=p_{k}(x)^{\prime} J_{\ell}^{-1}(\alpha) \mathbb{G}_{n}\left[v_{i} p_{i} \varphi_{i \ell}(\alpha)\right]+\bar{R}_{\ell}(x, \alpha)
$$

where $p_{i}=p_{k}\left(x_{i}\right), \sup _{\alpha \in \mathcal{A}}\left\|\bar{R}_{\ell}\left(x_{i}, \alpha\right)\right\|_{\mathbb{P}_{n}, 2} \rightarrow_{P} 0$, and $\left(x_{i}, z_{i}, \varphi_{i \ell}\right)$ are i.i.d. random elements.

C3 (Design Conditions). The score function $\varphi_{i \ell}(\alpha)$ is mean zero conditional on $x_{i}, z_{i}$ and has uniformly bounded fourth moment conditional on $x_{i}, z_{i}$. The score function is smooth in mean-quartic sense: $\mathrm{E}\left[\left(\varphi_{i \ell}(\alpha)-\varphi_{i \ell}(\tilde{\alpha})\right)^{4} \mid x_{i}, z_{i}\right]^{1 / 2} \leq C\|\alpha-\tilde{\alpha}\|^{\gamma_{\varphi}}$ for some constants $C$ and $\gamma_{\varphi}>0$. Matrices $J_{\ell}(\alpha)$ exist and are uniformly Lipschitz over $\alpha \in \mathcal{A}$, a bounded and compact subset of $\mathbb{R}^{l}$, and $\sup _{\alpha \in \mathcal{A}}\left\|J_{\ell}^{-1}(\alpha)\right\|$ as well as the operator norms of matrices $\mathrm{E}\left[z_{i} z_{i}^{\prime}\right], \mathrm{E}\left[z_{i} p_{i}^{\prime}\right]$, and $\mathrm{E}\left[\left\|p_{i} p_{i}^{\prime}\right\|^{2}\right]$ are uniformly bounded in $k . \mathrm{E}\left[\left\|z_{i}\right\|^{6}\right]$ and $\mathrm{E}\left[\left\|x_{i}\right\|^{6}\right]$ are finite. $\mathrm{E}\left[\left\|\theta_{\ell}\left(x_{i}, \alpha\right)\right\|^{6}\right]$ is uniformly bounded in $\alpha$, and $\mathrm{E}\left[\left|\varphi_{i \ell}(\alpha)\right|^{4} x_{i}, z_{i}\right]$ is uniformly bounded in $\alpha$, 
$x$, and $z$. The functions $\theta_{\ell}(x, \alpha)$ are smooth, namely $\left|\theta_{\ell}(x, \alpha)-\theta_{\ell}(x, \tilde{\alpha})\right| \leq L(x)\|\alpha-\tilde{\alpha}\|^{\gamma_{\theta}}$ for some constant $\gamma_{\theta}>0$ and some function $L(x)$ with $\mathrm{E}\left[L(x)^{4}\right]$ bounded.

C4 (Growth Restrictions). When $k \rightarrow \infty$, $\sup _{x \in X}\left\|p_{k}(x)\right\| \leq \xi_{k}$, and the following growth condition holds on the number of series terms:

$$
\log ^{2} n\left(n^{-m / 4}+\sqrt{(k / n) \cdot \log n} \cdot \max _{i}\left\|z_{i}\right\| \wedge \xi_{k}\right)\left|\sqrt{\max _{i \leq n,} F_{1}^{4}}\right| \rightarrow_{\mathrm{P}} 0, \quad \xi_{k}^{2} \log ^{2} n / n \rightarrow 0,
$$

where $m$ is defined in Condition C1 above and $F_{1}$ is defined in Condition C5 below.

C5 (Complexity of Relevant Function Classes). The function set $\mathcal{F}_{1}=\left\{\varphi_{i \ell}(\alpha), \alpha \in \mathcal{A}, \ell=\right.$ $0,1\}$ has a square $\mathrm{P}$-integrable envelope $F_{1}$ and has a uniform covering $L_{2}$ entropy equivalent to that of a VC class. The function class $\mathcal{F}_{2} \supseteq\left\{\theta_{i \ell}(\alpha), \alpha \in \mathcal{A}, \ell=0,1\right\}$ has a square Pintegrable envelope $F_{2}$ for the case of fixed $k$ and bounded envelope $F_{2}$ for the case of increasing $k$, and has a uniform covering $L_{2}$ entropy equivalent to that of a $\mathrm{VC}$ class.

4.2.1. Discussion and verification of conditions. Assumptions C3 and C5 are common regularity conditions and they can be verified using standard arguments.

Condition C1 requires that the covariates $z_{i}$ be continuously distributed, which in turn assures that the support function is everywhere differentiable in $q \in \mathcal{S}^{d-1}$, see BM (Lemma A.8) and Lemma 3 in the Appendix. The assumption fails in the presence of discrete covariates. In this case, the identified set has exposed faces and therefore its support function is not differentiable in directions $q$ orthogonal to these exposed faces, see e.g., Bontemps, Magnac, and Maurin (2012, Section 3.1). When discrete covariates are present, Condition C1 can be met by adding to each discrete covariate a small amount of smoothly distributed noise as shown in Appendix B.4, i.e. by using a data jittering method. Adding noise gives "curvature" to the exposed faces, thereby guaranteeing that the identified set intersects its supporting hyperplane in a given direction at only one point, and is therefore differentiable, see Schneider (1993, Corollary 1.7.3). Lemma 8 in the Appendix shows that the distance between the true identified set and the set resulting from jittered covariates can be made arbitrarily small. In particular, it can be made less than or equal to a chosen constant $\delta>0$ by setting the variance of the smoothly distributed noise as a function of $\delta$ and of the moments of $\theta_{\ell}(x, \alpha), \ell=0,1$. One can then obtain a valid confidence set for the original (non data-jittered) set $B(\alpha)$ by taking the confidence set based on the jittered support function, and enlarging it by $\delta$. Lemma 8 shows that the conservative bias of this enlarged confidence set is a function of $\delta$ that can be estimated, so that inference can be made arbitrarily slightly conservative.

Condition $\mathrm{C} 2$ requires the estimates of the bounding functions to be asymptotically linear. In addition, it requires that the number of series terms grows fast enough for the remainder term to disappear. This requirement must be reconciled with Condition C4, which limits the rate at which the number of series terms can increase. We show below how to verify these two conditions in each of the examples of Section 3.

Example (Mean regression, continued). We begin with the simplest case of mean regression with interval valued outcome data. In this case, the bounding functions $\theta_{\ell}(\cdot)$ do not depend on $\alpha$, and we have $\widehat{\theta}_{\ell}(\cdot)=p_{k}(\cdot)^{\prime} \widehat{\vartheta}_{k, \ell}$ with $\widehat{\vartheta}_{k, \ell}=\left(P^{\prime} P\right)^{-1} P^{\prime} y_{\ell}$ and $P=\left[p_{k}\left(x_{1}\right), \ldots, p_{k}\left(x_{n}\right)\right]^{\prime}$. 
Let $\vartheta_{k, \ell}$ be the coefficients of a projection of $\mathrm{E}\left[y_{\ell} \mid x_{i}\right]$ on $P$, or pseudo-true values, so that $\vartheta_{k, \ell}=\left(P^{\prime} P\right)^{-1} P^{\prime} \mathrm{E}\left[y_{\ell} \mid x_{i}\right]$. We then have the following linearization for $\widehat{\theta}_{\ell}(\cdot)$

$$
\sqrt{n}\left(\widehat{\theta}_{\ell}(x)-\theta_{\ell}(x)\right)=\sqrt{n} p_{k}(x)\left(P^{\prime} P\right)^{-1} P^{\prime}\left(y_{\ell}-\mathrm{E}\left[y_{\ell} \mid x\right]\right)+\sqrt{n}\left(p_{k}(x)^{\prime} \vartheta_{k, \ell}-\theta_{\ell}(x)\right) .
$$

This is in the form of (4.1) with $J_{\ell}=P^{\prime} P, \varphi_{i \ell}=\left(y_{i \ell}-\mathrm{E}\left[y_{\ell} \mid x_{i}\right]\right)$, and $R_{\ell}(x, \alpha)=R_{\ell}(x)=$ $\sqrt{n}\left(p_{k}(x)^{\prime} \vartheta_{k, \ell}-\theta_{\ell}(x)\right)$. The remainder term is simply approximation error. Many results on the rate of approximation error are available in the literature. This rate depends on the choice of approximating functions, smoothness of $\theta_{\ell}(x)$, and dimension of $x$. When using polynomials as approximating function, if $\theta_{\ell}(x)=\mathrm{E}\left[y_{\ell} \mid x_{i}\right]$ is $s$ times differentiable with respect to $x$, and $x$ is $d$ dimensional, then (see e.g. Newey (1997) or Lorentz (1986))

$$
\sup _{x}\left|p_{k}(x)^{\prime} \vartheta_{k, \ell}-\theta_{\ell}(x)\right|=O\left(k^{-s / d}\right)
$$

In this case $\mathrm{C} 2$ requires that $n^{1 / 2} k^{-s / d} \rightarrow 0$, or that $k$ grows faster than $n^{\frac{d}{2 s}}$. Assumption C4 limits the rate at which $k$ can grow. This assumption involves $\xi_{k}$ and $\sup _{i}\left|\varphi_{i \ell}\right|$. The behavior of these terms depends on the choice of approximating functions and some auxiliary assumptions. With polynomials as approximating functions and the support of $x$ compact with density bounded away from zero, $\xi_{k}=O(k)$. If $y_{i \ell}-\mathrm{E}\left[y_{\ell} \mid x_{i}\right]$ has exponential tails, then $\sup _{i}\left|\varphi_{i \ell}\right|=O\left(2(\log n)^{1 / 2}\right)$. In this case, a sufficient condition to meet $\mathrm{C} 4$ is that $k=o\left(n^{1 / 3} \log ^{-6} n\right)$. Thus, we can satisfy both $\mathrm{C} 2$ and $\mathrm{C} 4$ by setting $k \propto n^{\gamma}$ for any $\gamma \in\left(\frac{d}{2 s}, \frac{1}{3}\right)$ under the assumption that $3 d \leq 2 s$. Notice that as usual in semiparametric problems, we require undersmoothing compared to the rate that minimizes mean-squared error, which is $\gamma=\frac{d}{d+2 s}$. Also, our assumption requires increasing amounts of smoothness as the dimension of $x$ increases.

We now discuss how to satisfy assumptions $\mathrm{C} 2$ and $\mathrm{C} 4$ more generally. Recall that in our examples, the series estimates of the bounding functions solve

$$
\widehat{\theta}_{\ell}(\cdot, \alpha)=\underset{\theta_{\ell} \in \mathcal{L}^{2}(X, \mathrm{P})}{\arg \min } \mathbb{E}_{n}\left[m\left(y_{i \ell}, \theta_{\ell}\left(x_{i}, \alpha\right), \alpha\right)\right]
$$

or $\widehat{\theta}_{\ell}(\cdot, \alpha)=p_{k}(\cdot)^{\prime} \widehat{\vartheta}_{k, \ell}$ with $\widehat{\vartheta}_{k, \ell}=\arg \min _{\vartheta} \mathbb{E}_{n}\left[m\left(y_{i \ell}, p_{k}\left(x_{i}\right)^{\prime} \vartheta, \alpha\right)\right]$. As above, let $\vartheta_{k, \ell}$ be the solution to $\vartheta_{k, \ell}=\arg \min _{\vartheta} \mathrm{E}\left[m\left(y_{i \ell}, p_{k}\left(x_{i}\right)^{\prime} \vartheta, \alpha\right)\right]$. We show that the linearization in $\mathrm{C} 2$ holds by writing

$$
\sqrt{n}\left(\widehat{\theta}_{\ell}(x, \alpha)-\theta_{\ell}(x, \alpha)\right)=\sqrt{n} p_{k}(x)\left(\widehat{\vartheta}_{k, \ell}-\vartheta_{k, \ell}\right)+\sqrt{n}\left(p_{k}(x)^{\prime} \vartheta_{k, \ell}-\theta_{\ell}(x, \alpha)\right) .
$$

The first term in (4.2) is estimation error. We can use the results of He and Shao (2000) to show that

$$
\left(\widehat{\vartheta}_{k, \ell}-\vartheta_{k, \ell}\right)=\mathbb{E}_{n}\left[J_{\ell}^{-1} p_{i} \psi_{i}\right]+o_{p}\left(n^{-1 / 2}\right),
$$

where $\psi$ denotes the derivative of $m\left(y_{i \ell}, p_{k}\left(x_{i}\right)^{\prime} \vartheta, \alpha\right)$ with respect to $\vartheta$.

The second term in 4.2 is approximation error. Standard results from approximation theory as stated in e.g. Chen (2007) or Newey (1997) give the rate at which the error from the best $L_{2}$-approximation to $\theta_{\ell}$ disappears. When $m$ is a least squares objective function, these results can be applied directly. In other cases, such as quantile or distribution regression, further work must be done. 
Example (Quantile regression with interval valued data, continued). The results of Belloni, Chernozhukov, Chetverikov, and Fernandez-Val (2018) can be used to verify our conditions for quantile regression. The conditions required to apply their results are as follows.

(Q.1) The data $\left\{\left(y_{i 0}, y_{i 1}, x_{i}\right), 1 \leq i \leq n\right\}$ are an i.i.d. sequence of real $(2+d)$-vectors, and $x \in \mathcal{X} \equiv[0,1]^{d}$.

(Q.2) For $\ell \in\{0,1\}$, the conditional density of $y_{\ell}$ given $x$ is bounded above by $\bar{f}$ uniformly over $y_{\ell} \in \mathcal{Y}$ and $x \in \mathcal{X}$; its derivative is continuous and bounded in absolute value from above by $\overline{f^{\prime}}$ uniformly in $y_{\ell} \in \mathcal{Y}$ and $x \in \mathcal{X}$. The conditional density $f_{y_{\ell} \mid X}\left(Q_{y_{\ell} \mid x}(\alpha \mid x) \mid x\right)$ is bounded away from zero uniformly in $\alpha \in \mathcal{A}$ and $x \in \mathcal{X}$.

(Q.3) For all $k$, the eigenvalues of $\mathrm{E}\left[p_{i} p_{i}^{\prime}\right]$ are uniformly bounded above and away from zero.

(Q.4) For some $C>0, Q_{y_{\ell} \mid x}$ belongs to the Hölder ball $\Omega(s, C, \mathcal{X})$ for all $\left.\alpha \in \mathcal{A}\right]^{11}$ If the approximating functions are polynomials, we require $s>d$; if the approximating functions are B-splines of order $s_{0}$, we require $\left(s \wedge s_{0}\right)>d$.

(Q.5) For some $a>0 \xi_{k}=O\left(k^{a}\right) ; k$ is chosen such that $k^{3} \xi_{k}^{2}=o\left(n^{1-\epsilon}\right)$ and $k^{-b+1}=o\left(n^{-\epsilon}\right)$ for some constant $\epsilon>0$ and with $b=s / d-1$ for polynomials and $b=\left(s \wedge s_{0}\right) / d$ for B-splines.

Under these assumptions, Lemma 1 in Belloni, Chernozhukov, Chetverikov, and FernandezVal (2018) shows that the approximation error satisfies

$$
\sup _{x \in \mathcal{X}, \alpha \in \mathcal{A}}\left|p_{k}(x)^{\prime} \vartheta_{k}(\alpha)-\theta_{\ell}(x, \alpha)\right| \lesssim k^{-b} .
$$

Theorem 2 of Belloni, Chernozhukov, Chetverikov, and Fernandez-Val (2018) then shows that C2 holds. Condition C4 also holds because for quantile regression $\psi_{i}$ is bounded, so C4 only requires $k^{1 / 2+a}(\log n)^{5 / 2}=o\left(n^{1 / 2}\right.$ ) (with $a=1$ for polynomials and $a=1 / 2$ for splines), which is implied by Q.5.

Example (Distribution regression, continued). In this example, $\theta_{\ell}(x, \alpha)=F_{y_{1-\ell} \mid x}(\alpha \mid x)$, $\ell=0,1$. As described above, the estimator solves $\widehat{\vartheta}_{k \ell}=\arg \min _{\vartheta} \mathbb{E}_{n}\left[m\left(y_{i \ell}, p_{k}\left(x_{i}\right)^{\prime} \vartheta, \alpha\right)\right]$, with

$$
m\left(y_{i \ell}, p_{k}\left(x_{i}\right)^{\prime} \vartheta, \alpha\right)=-1\left\{y_{i \ell}<\alpha\right\} \log \Phi\left(p_{k}\left(x_{i}\right)^{\prime} \vartheta\right)-1\left\{y_{i \ell} \geq \alpha\right\} \log \left(1-\Phi\left(p_{k}\left(x_{i}\right)^{\prime} \vartheta\right)\right)
$$

for some known distribution function $\Phi$. We must show that (i) the estimation error, $\widehat{\vartheta}_{k, \ell}-\vartheta_{k, \ell}$, can be linearized; and that (ii) the approximation error, $p_{k}(x) \vartheta_{k, \ell}-\theta_{\ell}(x, \alpha)$, vanishes at rate $o\left(n^{-1 / 2}\right)$. We accomplish this by verifying the conditions of He and Shao (2000) to show that $\left(\widehat{\vartheta}_{k, \ell}-\vartheta_{k, \ell}\right)$ can be linearized, and by verifying the conditions of Hirano, Imbens, and Ridder (2003) to show that the approximation bias is of the correct order. We let $\mathcal{A}_{0} \subset \mathcal{A}$ denote the set of values for $\alpha$ that the researcher is interested in. The conditions required to apply their results are as follows.

(D.1) The distribution function $\Phi$ is smooth; its associated probability density function $\phi$ has a bounded derivative.

(D.2) The data $\left\{\left(y_{i 0}, y_{i 1}, x_{i}\right), 1 \leq i \leq n\right\}$ are an i.i.d. sequence of real $(2+d)$-vectors.

\footnotetext{
${ }^{11}$ See Belloni, Chernozhukov, Chetverikov, and Fernandez-Val (2018, p. 14) for a formal definition of the Hölder ball.
} 
(D.3) $\mathcal{X}=[0,1]^{d}$. The density function of $x$ is uniformly bounded away from zero on $\mathcal{X}$.

(D.4) For all $k$, the eigenvalues of $\mathrm{E}\left[p_{i} p_{i}^{\prime}\right]$ are uniformly bounded above and away from zero. For some $a>0, \xi_{k}=O\left(k^{a}\right)$ and $k=o\left((n / \log n)^{1 / 2}\right)$.

(D.5) For each $\ell \in\{0,1\}$ and for some $C>0, \theta_{\ell}(x, \alpha)$ belongs to the Hölder ball $\Omega(s, C, \mathcal{X})$ for all $\alpha \in \mathcal{A}$, with $s / d \geq 4$.

(D.6) For each $\ell \in\{0,1\}, \theta_{\ell}(x, \alpha)$ is bounded away from 0 and from 1, uniformly in $\alpha \in \mathcal{A}_{0}$.

Adopting the notation of $\mathrm{He}$ and Shao (2000), in this example we have that the derivative of $m\left(y_{i \ell}, p_{k}\left(x_{i}\right)^{\prime} \vartheta, \alpha\right)$ with respect to $\vartheta$ is

$$
\psi\left(y_{i \ell}, x_{i}, \vartheta\right)=-\left(\frac{1\left\{y_{i \ell}<\alpha\right\}}{\Phi\left(p_{k}\left(x_{i}\right)^{\prime} \vartheta\right)}-\frac{1\left\{y_{i \ell} \geq \alpha\right\}}{1-\Phi\left(p_{k}\left(x_{i}\right)^{\prime} \vartheta\right)}\right) \phi\left(p_{k}\left(x_{i}\right)^{\prime} \vartheta\right) p_{k}\left(x_{i}\right) .
$$

Because for each $\ell \in\{0,1\} m\left(y_{i \ell}, p_{k}\left(x_{i}\right)^{\prime} \vartheta, \alpha\right)$ is a smooth function of $\vartheta, \mathbb{E}_{n} \psi\left(y_{i \ell}, x_{i}, \widehat{\vartheta}_{k, \ell}\right)=$ 0, and conditions C.0 and C.2 in He and Shao (2000) hold. By condition D.1, $\psi$ is Lipschitz in $\vartheta$, and we have the bound

$$
\left\|\eta_{i \ell}(\vartheta, \tau)\right\|^{2} \lesssim\left\|p_{k}\left(x_{i}\right)\right\|^{2}\|\tau-\vartheta\|^{2}
$$

where $\eta_{i \ell}(\vartheta, \tau)=\psi\left(y_{i \ell}, x_{i}, \vartheta\right)-\psi\left(y_{i \ell}, x_{i}, \tau\right)-E \psi\left(y_{i \ell}, x_{i}, \vartheta\right)+E \psi\left(y_{i \ell}, x_{i}, \tau\right)$. By condition D.4, which is satisfied by polynomials with $a=1$ or by splines with $a=1 / 2$, condition C.1 in He and Shao (2000) holds. Differentiability of $\phi$ and our assumption C3 are sufficient for C.3 in He and Shao (2000). Finally, conditions C.4 and C.5 in He and Shao (2000) hold with $A(n, k)=k$ because

$$
\left|s^{\prime} \eta_{i \ell}(\vartheta, \tau)\right|^{2} \lesssim\left|s^{\prime} p_{k}\left(x_{i}\right)\right|^{2}\|\tau-\vartheta\|^{2}
$$

and $\mathrm{E}\left[\left|s^{\prime} p_{k}\left(x_{i}\right)\right|^{2}\right]$ is uniformly bounded for $s \in \mathcal{S}^{k-1}:=\left\{q \in \mathbb{R}^{k}:\|q\|=1\right\}$ for all $k$ when the series functions are orthonormal. Applying Theorem 2.2 of He and Shao (2000), we obtain the desired linearization if $k=o\left((n / \log n)^{1 / 2}\right)$.

The results of Hirano, Imbens, and Ridder (2003) can be used to show that the approximation bias is sufficiently small. All conditions required by Lemma 1 in Hirano, Imbens, and Ridder (2003) are satisfied by our set of conditions D.1-D.6, and therefore for $\Phi$ given by the logistic distribution we have for each $\ell \in\{0,1\}$

$$
\sup _{x \in \mathcal{X}, \alpha \in \mathcal{A}}\left|\Phi\left(p_{k}(x) \vartheta_{k, \ell}\right)-\Phi\left(\theta_{\ell}(x, \alpha)\right)\right|=O\left(k^{-s /(2 d)} \xi_{k}\right),
$$

which implies that

$$
\sup _{x \in \mathcal{X}, \alpha \in \mathcal{A}}\left|p_{k}(x) \vartheta_{k, \ell}-\theta_{\ell}(x, \alpha)\right|=O\left(k^{-s /(2 d)} \xi_{k}\right) .
$$

This result is only for the logistic link function, but it can easily be adapted for any link function with first derivative bounded from above and second derivative bounded away from zero. We need the approximation error to be $o\left(n^{-1 / 2}\right)$. For this, it suffices to have

$$
k^{-s /(2 d)} \xi_{k} n^{1 / 2}=o(1) .
$$

Given condition D.4. it suffices to have $k \propto n^{\gamma}$ for $\gamma>\frac{d}{s-2 a d}$. 
To summarize, condition $\mathrm{C} 2$ can be met by having $k \propto n^{\gamma}$ for any $\gamma \in\left(\frac{d}{s-2 a d}, \frac{1}{2}\right)$, and, as in the mean and quantile regression examples above, condition $\mathrm{C} 4$ will be met if $\gamma<\frac{1}{1+2 a}$.

4.3. Theoretical Results. In order to state the result we define

$$
\begin{aligned}
h_{i k}(t):= & q^{\prime} \Sigma \mathrm{E}\left[z_{i} p_{i}^{\prime} 1\left\{q^{\prime} \Sigma z_{i}>0\right\}\right] J_{1}^{-1}(\alpha) p_{i} \varphi_{i 1}(\alpha) \\
+ & q^{\prime} \Sigma \mathrm{E}\left[z_{i} p_{i}^{\prime} 1\left\{q^{\prime} \Sigma z_{i}<0\right\}\right] J_{0}^{-1}(\alpha) p_{i} \varphi_{i 0}(\alpha) \\
& -\quad q^{\prime} \Sigma x_{i} z_{i}^{\prime} \Sigma \mathrm{E}\left[z_{i} w_{i, q^{\prime} \Sigma}(\alpha)\right] \\
+ & q^{\prime} \Sigma z_{i} w_{i, q^{\prime} \Sigma}(\alpha) .
\end{aligned}
$$

We begin by providing limit theory results for the support function process. Below, $\mathbb{G}_{n}\left[h_{k}(t)\right]:=$ $\frac{1}{\sqrt{n}} \sum_{i=1}^{n}\left(h_{i k}(t)-\mathrm{E}_{k}(t)\right)$.

Theorem 1 (Limit Theory for Support Function Process). The support function process $S_{n}(t)=\sqrt{n}\left(\widehat{\sigma}_{\widehat{\theta}, \widehat{\Sigma}}-\sigma_{\theta, \Sigma}\right)(t)$, where $t=(q, \alpha) \in T$, admits the approximation $S_{n}(t)=$ $\mathbb{G}_{n}\left[h_{k}(t)\right]+o_{\mathrm{P}}(1)$ in $\ell^{\infty}(T)$. Moreover, the support function process admits an approximation

$$
S_{n}(t)=\mathbb{G}\left[h_{k}(t)\right]+o_{\mathrm{P}}(1) \text { in } \ell^{\infty}(T),
$$

where the process $\mathbb{G}\left[h_{k}(t)\right]$ is a tight P-Brownian bridge in $\ell^{\infty}(T)$ with covariance function $\Omega_{k}\left(t, t^{\prime}\right)=\mathrm{E}\left[h_{k}(t) h_{k}\left(t^{\prime}\right)\right]-\mathrm{E}\left[h_{k}(t)\right] \mathrm{E}\left[h_{k}\left(t^{\prime}\right)\right]$ that is uniformly Holder on $T \times T$ uniformly in $k$, and is uniformly non-degenerate in $k$. These bridges are stochastically equicontinuous with respect to the $L_{2}$ pseudo-metric $\rho_{2}\left(t, t^{\prime}\right)=\left[\mathrm{E}\left[h(t)-h\left(t^{\prime}\right)\right]^{2}\right]^{1 / 2} \lesssim\left\|t-t^{\prime}\right\|^{c}$ for some $c>0$ uniformly in $k$. The sequence $\mathbb{G}\left[h_{k}(t)\right]$ does not necessarily converge weakly under $k \rightarrow \infty$; however, each subsequence has a further convergent subsequence converging to a tight Gaussian process in $\ell^{\infty}(T)$ with a non-degenerate covariance function. Furthermore, the canonical distance between the law of the support function process $S_{n}(t)$ and the law of $\mathbb{G}\left[h_{k}(t)\right]$ in $\ell^{\infty}(T)$ approaches zero, namely $\sup _{g \in B L_{1}\left(\ell^{\infty}(T),[0,1]\right)}\left|\mathrm{E}\left[g\left(S_{n}\right)\right]-\mathrm{E}\left[g\left(\mathbb{G}\left[h_{k}\right]\right)\right]\right| \rightarrow 0$.

Our second step is to provide inference results for various statistics based on the support function process. Formally, we consider these statistics as mappings $f: \ell^{\infty}(T) \rightarrow \mathbb{R}$ from the possible values $s$ of the support function process $S_{n}$ to the real line. Examples include:

- a support function evaluated at $t \in T, f(s)=s(t)$,

- a Kolmogorov statistic, $f(s)=\sup _{t \in T_{0}}|s(t)| / \varpi(t)$,

- a directed Kolmogorov statistic, $f(s)=\sup _{t \in T_{0}}\{-s(t)\}_{+} / \varpi(t)$,

- a Cramer-Von-Mises statistic, $f(s)=\int_{T} s^{2}(t) / \varpi(t) d \nu(t)$,

where $T_{0}$ is a subset of $T, \varpi$ is a continuous and uniformly positive weighting function, and $\nu$ is a probability measure over $T$ whose support is $T^{12}$ Allowing for a weighting function $\varpi$ is important because, as shown in Chernozhukov, Kocatulum, and Menzel (2015), it enforces either exact or first-order equivariance of the statistics to transformations of parameters, yielding more powerful inference. More generally we can consider any continuous function $f$ such that $f(Z)$ (a) has a continuous distribution function when $Z$ is a tight Gaussian process with non-degenerate covariance function and (b) $f\left(\zeta_{n}+c\right)-f\left(\zeta_{n}\right)=o(1)$ for any

\footnotetext{
${ }^{12}$ Observe that test statistics based on the (directed) Hausdorff distance (see, e.g., BM) are special cases of the (directed) Kolmogorov statistics above.
} 
$c=o(1)$ and any $\left\|\zeta_{n}\right\|=O(1)$. Denote the class of such functions $\mathcal{F}_{c}$ and note that the examples mentioned above belong to this class by the results of Davydov, Lifshits, and Smorodina (1998).

Theorem 2 (Limit Inference on Support Function Process). For any $\widehat{c}_{n}=c_{n}+o_{\mathrm{P}}(1)$ and $c_{n}=O_{\mathrm{P}}(1)$ and $f \in \mathcal{F}_{c}$ we have

$$
\mathrm{P}\left\{f\left(S_{n}\right) \leq \widehat{c}_{n}\right\}-\mathrm{P}\left\{f\left(\mathbb{G}\left[h_{k}\right]\right) \leq c_{n}\right\} \rightarrow 0 .
$$

If $c_{n}(1-\tau)$ is the $(1-\tau)$-quantile of $f\left(\mathbb{G}\left[h_{k}\right]\right)$ and $\widehat{c}_{n}(1-\tau)=c_{n}(1-\tau)+o_{\mathrm{P}}(1)$ is any consistent estimate of this quantile, then

$$
\mathrm{P}\left\{f\left(S_{n}\right) \leq \widehat{c}_{n}(1-\tau)\right\} \rightarrow 1-\tau .
$$

Next we provide limit theory results for the bootstrap support function process. Let $\mathrm{P}^{e}$ denote the probability measure conditional on the data, $e_{i}^{o}=e_{i}-1$, and $h_{k}^{o}=h_{k}-\mathrm{E}\left[h_{k}\right]$.

Theorem 3 (Limit Theory for the Bootstrap Support Function Process). The bootstrap support function process $\tilde{S}_{n}(t)=\sqrt{n}\left(\widetilde{\sigma}_{\widetilde{\theta}, \widetilde{\Sigma}}-\widehat{\sigma}_{\widehat{\theta}, \widehat{\Sigma}}\right)(t)$, where $t=(q, \alpha) \in T$, admits the following approximation conditional on the data: $\widetilde{S}_{n}(t)=\mathbb{G}_{n}\left[e_{i}^{o} h_{k}^{o}(t)\right]+o \mathrm{Pe}(1)$ in $\ell^{\infty}(T)$ in probability $\mathrm{P}$. Moreover, the bootstrap support function process admits an approximation conditional on the data:

$$
\widetilde{S}_{n}(t)=\widetilde{\mathbb{G}\left[h_{k}(t)\right]}+o_{\mathrm{P} e}(1) \text { in } \ell^{\infty}(T) \text {, in probability } \mathrm{P},
$$

where $\widetilde{\mathbb{G}\left[h_{k}\right]}$ is a sequence of tight $\mathrm{P}$-Brownian bridges in $\ell^{\infty}(T)$ with the same distributions as the processes $\mathbb{G}\left[h_{k}\right]$ defined in Theorem 1 , and independent of $\mathbb{G}\left[h_{k}\right]$. Furthermore, the canonical distance between the law of the bootstrap support function process $\widetilde{S}_{n}(t)$ conditional on the data and the law of $\mathbb{G}\left[h_{k}(t)\right]$ in $\ell^{\infty}(T)$ approaches zero, namely $\sup _{g \in B L_{1}\left(\ell^{\infty}(T),[0,1]\right)}\left|\mathrm{E}_{\mathrm{P} e}\left[g\left(\widetilde{S}_{n}\right)\right]-\mathrm{E}\left[g\left(\mathbb{G}\left[h_{k}\right]\right)\right]\right| \rightarrow_{P} 0$.

We conclude by establishing that inference can be carried out using critical values estimated using our proposed Bayesian bootstrap procedure.

Theorem 4 (Bootstrap Inference on the Support Function Process). For any $c_{n}=O_{\mathrm{P}}(1)$ and $f \in \mathcal{F}_{c}$ we have

$$
\mathrm{P}\left\{f\left(S_{n}\right) \leq c_{n}\right\}-\mathrm{P}^{e}\left\{f\left(\widetilde{S}_{n}\right) \leq c_{n}\right\} \rightarrow_{P} 0 .
$$

In particular, if $\widetilde{c}_{n}(1-\tau)$ is the $(1-\tau)$-quantile of $f\left(\widetilde{S}_{n}\right)$ under $\mathrm{P}^{e}$, then

$$
\mathrm{P}\left\{f\left(S_{n}\right) \leq \widetilde{c}_{n}(1-\tau)\right\} \rightarrow_{P} 1-\tau .
$$

4.4. Intersection bounds. Suppose we are interested in a function $\theta: \mathcal{X} \rightarrow \mathbb{R}$ with bounds of the form

$$
\theta_{0}(x, \alpha, v) \leq \theta(x, \alpha) \leq \theta_{1}(x, \alpha, v) \forall v \in \mathcal{V}_{x} .
$$

Our example of quantile regression with selection and an exclusion restriction has this form. Our results can easily be applied to this case. 
First, note that taking the intersection over $v$ and then forming the set of best linear approximations is the same as forming the set of best linear approximations for each $v$, and then intersecting the sets of best linear approximations. To see this, let

$$
\begin{aligned}
B(\alpha) & =\left\{\beta \in \underset{b}{\arg \min } \mathrm{E}\left[\phi(x, \alpha)-x^{\prime} b\right]: \theta_{0}(x, \alpha, v) \leq \phi(x, \alpha) \leq \theta_{1}(x, \alpha, v) x-a . s . \forall v \in \mathcal{V}_{x}\right\} \\
& =\left\{\beta \in \underset{b}{\arg \min } \mathrm{E}\left[\phi(x, \alpha)-x^{\prime} b\right]: \sup _{v \in \mathcal{V}_{x}} \theta_{0}(x, v, \alpha) \leq \phi(x, \alpha) \leq \inf _{v \in \mathcal{V}_{x}} \theta_{1}(x, v, \alpha) x-a . s .\right\},
\end{aligned}
$$

and let

$$
B(\alpha, v)=\left\{\beta \in \underset{b}{\arg \min } \mathrm{E}\left[\phi(x, \alpha)-x^{\prime} b\right]: \theta_{0}(x, \alpha, v) \leq \phi(x, \alpha) \leq \theta_{1}(x, \alpha, v) x-a . s .\right\},
$$

Recall that we are assuming without loss of generality that $\mathcal{V}_{x}=\mathcal{V}$ for all $x{ }^{13}$ and that $\mathcal{V}$ is finite. It is then trivial to verify that $B(\alpha)=\cap_{v \in \mathcal{V}} B(\alpha, v)$ because all sets $B(\alpha, v)$ are parallel shifts of each other. Additionally, if $\sigma(q, \alpha)$ is the support function of $B(\alpha)$ and $\sigma(q, \alpha, v)$ is the support function of $B(\alpha, v)$, then

$$
\sigma(q, \alpha)=\inf _{\sum_{v \in \mathcal{V}} q_{v}=q}\left\{\sum_{v \in \mathcal{V}} \sigma\left(q_{v}, \alpha, v\right)\right\}
$$

see Rockafellar (1970, Corollary 16.4.1).

To apply theorems 1,4, assume conditions C1 C5 hold for each $v \in \mathcal{V}$, with $(x, v) \in \mathcal{X} \times \mathcal{V}$ replacing $x \in \mathcal{X}$ in each condition. Then denoting by $q_{\mathcal{V}}:=\left[q_{v}, v \in \mathcal{V}\right]$, theorems 1 , 4 apply to $S_{n}(q \mathcal{V}, \alpha, \mathcal{V}):=\sqrt{n}\left[\sum_{v \in \mathcal{V}}\left(\widehat{\sigma}\left(q_{v}, \alpha, v\right)-\sigma\left(q_{v}, \alpha, v\right)\right)\right]$. On the other hand, the support function process for $\widehat{\sigma}(q, \alpha)$ is

$$
S_{n}(q, \alpha)=\sqrt{n}\left(\inf _{q_{\mathcal{V}} \in Q_{\mathcal{V}}} \sum_{v \in \mathcal{V}} \widehat{\sigma}\left(q_{v}, \alpha, v\right)-\inf _{q_{\mathcal{V}} \in Q_{\mathcal{V}}} \sum_{v \in \mathcal{V}} \sigma\left(q_{v}, \alpha, v\right)\right)
$$

where $Q_{\mathcal{V}}:=\left\{q_{v}, v \in \mathcal{V}: \sum_{v \in \mathcal{V}} q_{v}=q\right\}$. Note that

$$
-\sup _{q_{\mathcal{V}} \in Q_{\mathcal{V}}}\left|\sqrt{n}\left[\sum_{v \in \mathcal{V}}\left(\widehat{\sigma}\left(q_{v}, \alpha, v\right)-\sigma\left(q_{v}, \alpha, v\right)\right)\right]\right| \leq S_{n}(q, \alpha) \leq \sup _{q_{\mathcal{V}} \in Q_{\mathcal{V}}}\left|\sqrt{n}\left[\sum_{v \in \mathcal{V}}\left(\widehat{\sigma}\left(q_{v}, \alpha, v\right)-\sigma\left(q_{v}, \alpha, v\right)\right)\right]\right|
$$

This inequality along with theorems 2 and 4 allows for conservative inference for many statistics of interest. For example,

$$
\mathrm{P}\left\{\sup _{(q, \alpha) \in T_{0}}\left|S_{n}(t)\right| \leq c_{n}\right\} \geq \mathrm{P}\left\{\sup _{(q, \alpha) \in T_{0}} \sup _{q \mathcal{V} \in Q_{\mathcal{V}}}\left|\sqrt{n}\left[\sum_{v \in \mathcal{V}}\left(\widehat{\sigma}\left(q_{v}, \alpha, v\right)-\sigma\left(q_{v}, \alpha, v\right)\right)\right]\right| \leq c_{n}\right\},
$$

and since $f: \ell^{\infty}(T \times \mathcal{V}) \rightarrow \mathbb{R}$ defined by $f\left(S_{n}\right)=\sup _{(q, \alpha) \in T_{0}} \sup _{q_{\mathcal{V}} \in Q_{\mathcal{V}}}\left|S_{n}(q \mathcal{V}, \alpha, \mathcal{V})\right|$ is in $\mathcal{F}_{c}$, theorems 2 and 4 apply.

This procedure will generally be conservative because it treats uncertainty in $\widehat{\sigma}(q, v, \alpha)$ for $v$ far from the minimum as though it is as relevant as uncertainty in $\widehat{\sigma}(q, v, \alpha)$ near its minimum. More powerful inference can be achieved by employing either the precision

\footnotetext{
${ }^{13}$ This is without loss of generality because we can assign $\theta_{0}(x, v)=-\infty$ and $\theta_{1}(x, v)=\infty$ for $v \notin \mathcal{V}_{x}$, and this change does not affect $B(\alpha)$.
} 
correction procedure of Chernozhukov, Lee, and Rosen (2013) or the generalized moment selection approach of Andrews and Shi (2013).

In the special case that $v_{0}^{*}$ is the unique maximizer of $\theta_{0}(x, v, \alpha)$ and $v_{1}^{*}$ is the unique minimizer of $\theta_{1}(x, v, \alpha)$ over $v \in \mathcal{V}$, we show in Lemma 7 in the Appendix that condition $\mathrm{C} 2$ is satisfied by an estimator of the bounding functions equal to the maximum (minimum)

of $p_{k}(x, v)^{\prime} \widehat{\vartheta}_{k, \ell}^{x v}$ over $v \in \mathcal{V}$. We then have that all our conditions apply, and exact inference can be carried out working with the set $B(\alpha)$ as defined in $(4.3)$.

\section{Applichtion: the Gender Wage gap and selection}

An important question in labor economics is whether the gender wage gap is shrinking over time. Blau and Kahn (1997) and Card and DiNardo (2002), among others, have noted the coincidence between a rise in within-gender inequality and a fall in the gender wage gap over the last 40 years. Mulligan and Rubinstein (2008) observe that the growing wage inequality within gender should induce females to invest more in productivity. In turn, able females should differentially be pulled into the workforce. Motivated by this observation, they use Heckman's two-step estimator on repeated Current Population Survey cross-sections in order to compute relative wages for women since 1970, holding skill composition constant. They find that in the 1970s selection into the female workforce was negative, while in the 1990s it was positive. Moreover, they argue that the majority of the reduction in the gender gap can be attributed to the changes in the female workforce composition. In particular, the OLS estimates of the log-wage gap suggest that it has fallen from -0.419 in the 1970 s to -0.256 in the 1990 s, while the Heckman two step estimates suggest that once one controls for skill composition, the wage gap is -0.379 in the $1970 \mathrm{~s}$ and -0.358 in the 1990s. Based on these results, Mulligan and Rubinstein (2008) conclude that the wage gap has not shrunk over the last 40 years. Rather, the behavior of the OLS estimates can be explained by a switch from negative to positive selection into female labor force participation. See Blau and Kahn (2017) for a thorough review of this literature.

In what follows, we address the same question as Mulligan and Rubinstein (2008), using the same data, the same variables and the same instruments as in their original study. However, we use our method to estimate bounds on the quantile gender wage gap without assuming a parametric form of selection or a strong exclusion restriction. We compare conditional quantiles that ignore the selection effect, with the bounds on these quantiles that one obtains when taking selection into account. Our results indicate the presence of a marked gender wage gap at quantiles below the 0.4, especially for the subsample of lower educated and of married individuals. However, we are unable to reject that the gender wage gap declined over the period in question. This suggests that the instruments may not be sufficiently strong to yield tight bounds and that there may not be enough information in the data to conclude that the gender gap has or has not declined from 1975 to 1999 without strong functional form assumptions.

5.1. Setup. The Mulligan and Rubinstein (2008) setup relates log-wage to covariates in a linear model as follows:

$$
\log w=x^{\prime} \beta+\varepsilon,
$$


TABLE 1. Gender wage gap estimates

\begin{tabular}{c|ccccc} 
& OLS & 2 -step & QR(0.5) & Low & High \\
\hline $1975-1979$ & -0.408 & -0.360 & -0.522 & -1.242 & 0.588 \\
& $(0.003)$ & $(0.013)$ & $(0.003)$ & $(0.016)$ & $(0.061)$ \\
$1995-1999$ & -0.268 & -0.379 & -0.355 & -0.623 & 0.014 \\
& $(0.003)$ & $(0.013)$ & $(0.003)$ & $(0.012)$ & $(0.010)$
\end{tabular}

Estimates of the gender wage gap (female - male) conditional on having average characteristics. Column 1: OLS estimates of the average gender gap. Column 2: Heckman two-step estimates. Column 3: quantile regression estimates of the median gender wage gap ignoring selection. Columns 4-5: estimates of bounds on the median wage gap that account for selection. Standard errors are shown in parentheses (for the bounds, these were calculated using the Bayesian bootstrap described in Section 4.1.3).

wherein $x$ includes marital status, years of education, potential experience, potential experience squared, and region dummies, as well as their interactions with an indicator for gender which takes the value 1 if the individual is female, and zero otherwise. They model selection as in the following equation:

$$
u=1\left\{z^{\prime} \gamma+\eta>0\right\},
$$

where $z=\left[\begin{array}{ll}x & \tilde{z}\end{array}\right], \tilde{z}$ is marital status interacted with indicators for having zero, one, two, or more than two children, and $\eta$ is the unobservable.

For each quantile, we estimate bounds for the gender wage gap utilizing our method. The bound equations we use are given by $\theta_{\ell}(x, \alpha, v)=p_{k}(x, v)^{\prime} \vartheta_{k, \ell}^{x v}(\alpha)$, where $p_{k}(x, v)=$ $\left[\begin{array}{lll}x & v & w\end{array}\right], v$ are indicators for the number of children, and $w$ consists of years of education squared, potential experience cubed, and education $\times$ potential experience, and $v$ interacted with marital status. In our data, the maximizer (minimizer) over $x \in \mathcal{V}$ of the lower (upper) bounding function is unique, therefore exact inference can be carried out as described at the end of Section 4.4. Hence, after taking the intersection of the bounds over the excluded variables $v$, our estimated bounding functions are simply the minimum or maximum over $v$ of $p_{k}(x, v)^{\prime} \vartheta_{k, \ell}^{x v}(\alpha)$.

5.2. Results. Let $\bar{x}_{f}$ be a female with average (unconditional on gender or year) characteristics and $\bar{x}_{m}$ be a male with average (unconditional on gender or year) characteristics. In what follows, we report the predicted gender wage gap for someone with average characteristics, $\left(\bar{x}_{f}-\bar{x}_{m}\right) \beta(\alpha)$. The first two columns of Table 1 reproduce the results of Mulligan and Rubinstein (2008). The first column shows the gender wage gap estimated by ordinary least squares. The second column shows estimates from Heckman's two-step selection correction. The OLS estimates show a decrease in the wage gap, while the Heckman selection estimates show no change. The third column shows estimates of the median gender wage gap from quantile regression. Like OLS, quantile regression shows a decrease in the gender wage gap. The final two columns show bounds on the median gender wage gap that account for selection. The bounds are wide, especially in the 1970s, and cover zero, indicating that the data alone do not contain sufficient information to sign the median gender wage gap.

Figure 5.1 shows the estimated quantile gender wage gaps in the 1970s and 1990s. The solid black line shows the quantile gender wage gap when selection is ignored. In both the 
Figure 5.1. Bounds at Quantiles for full sample

1975-1979

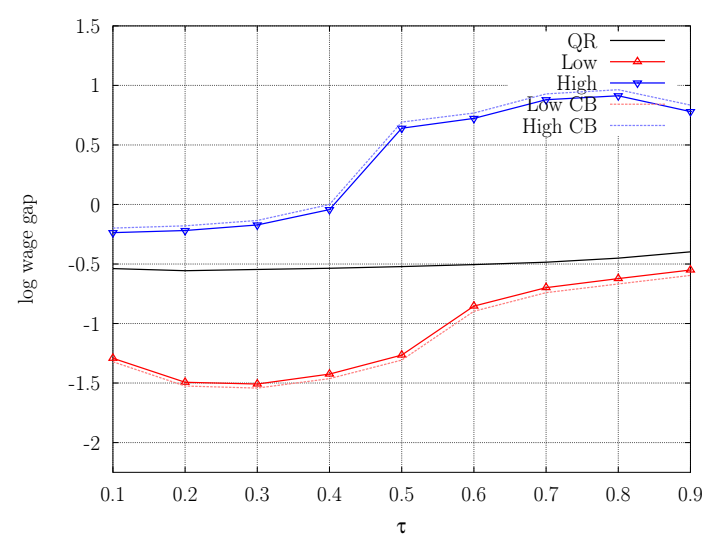

1995-1999

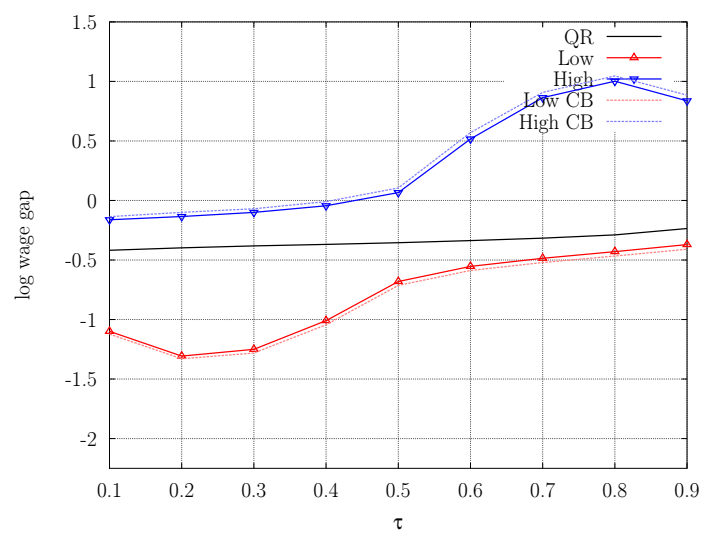

Estimated quantile gender wage gap (female - male) conditional on having average characteristics. The solid black line shows the quantile gender wage gap when selection is ignored. The blue and red lines with upward and downward pointing triangles show upper and lower bounds that account for employment selection for females. The dashed lines represent a uniform $90 \%$ confidence region for the bounds.

1970s and 1990s, the gender wage gap is larger for lower quantiles. At all quantiles the gap in the 1990s is about $40 \%$ smaller than in the 1970s. However, this result should be interpreted with caution because it ignores selection into the labor force.

The blue line with downward pointing triangles and the red line with upward pointing triangles show our estimated bounds on the gender wage gap after accounting for selection. The dashed lines represent a uniform 90\% confidence region. In both the 1970s and 1990s, the upper bound lies below zero for low quantiles, roughly up to the 0.4. This means that the low quantiles of the distribution of wages conditional on having average characteristics are lower for women than for men. This difference exists even if we allow for the most extreme form of selection (subject to our exclusion restriction) into the labor force for women, thereby yielding a very robust result. For quantiles at or above the median, our estimated upper bound lies above zero and our lower bound lies below zero. Thus, high quantiles of the distribution of wages conditional on average characteristics could be either higher or lower for women than for men, depending on the true pattern of selection.

The bounds in Figure 5.1 are tighter for the 1990s than for the 1970s. This reflects higher female labor force participation in the 1990s. For all quantiles, there is considerable overlap between the identification region in the 1970s and in the 1990s. Therefore, we conclude that the data does not contain enough information to sign the changes in the gender wage gap over time without additional assumptions.

To further explore what can be learned about the change over time in the gender wage gap of specific demographic groups, Figures C.1 C.4 in Appendix C show the estimated quantile bounds conditional on being in these subgroups. That is, rather than reporting the gender wage gap for someone with average characteristics, these figures show the gender 
wage gap for someone with average subgroup characteristics (e.g., unconditional on gender or year, but conditional on subgroup: marital status and education level). To generate these figures, the entire model was re-estimated using only observations within each subgroup.

Figures C.1 and C.2 show the results for singles and people with at least 16 years of education. The results are broadly similar to the results for the full sample. There is robust evidence of a gap at low quantiles, although it is only marginally significant for the highly educated in the 1990s. As expected, the bounds are tighter than the full sample bounds because these subgroups have higher labor force participation. For comparison, Figure C.3 shows the results for people with no more than a high school degree, and Figure C.4 shows results for singles with at least a college degree. Across all subgroups, the bounds on the gender wage gap at higher quantiles and on the change in the gap over time continue to be wide, suggesting the need to augment the model with additional assumptions.

5.2.1. With restrictions on selection. Blundell, Gosling, Ichimura, and Meghir (2007) previously studied changes in the distribution of wages in the UK. They perform partial identification analysis by estimating quantile bounds that account for selection. Similarly to what we have reported, Blundell, Gosling, Ichimura, and Meghir (2007) find that the estimated bounds are quite wide. As a result, they explore various restrictions to tighten the bounds. One such restriction posits that the distribution of wages for the employed stochastically dominates the distribution of wages for those not working. This implies that the observed quantiles of wages conditional on employment are an upper bound for the quantiles of wages not conditional on employment.

Figure 5.2 shows the results for the full sample of our approach imposing stochastic dominance, while Figure C.5 in Appendix C shows the results for the subsample of highly educated singles. Stochastic dominance implies that the upper bound on each quantile coincides with the corresponding quantile regression estimate that ignores selection. The assumption has substantial identification power in our data. It yields strong evidence of a gender wage gap at all quantiles in both the 1970s and 1990s, for both the full sample and the subsample of highly educated singles. Moreover, the assumption has sufficient identifying power when combined with our data to yield informative results on the change in the gender wage gap over time. Figure 5.3 shows the estimated change, both for the full sample and for the subsample of highly educated singles. For the full sample, the estimated bounds include zero at low and moderate quantiles. At the 0.8 and higher quantiles, there is evidence that the gender wage gap decreased by approximately $0.15 \log$ dollars. For highly educated singles, there is evidence that the gender wage gap did not increase at the 0.7 and higher quantiles, although -in part due to the smaller size of the subgroup- the confidence bands are relatively wide and hence consistent with an increase.

While these results allow one to draw some strong conclusions, the assumption of positive selection into employment is not innocuous. It may be violated if there is a strong positive correlation between potential wages and reservation wages. This may be the case if there is positive assortative matching in the marriage market. Women with high potential wages could marry men with high wages, making these high potential wage women less likely to work. Also, the conclusion of Mulligan and Rubinstein (2008) that there was a switch from adverse selection into the labor market in the 1970s to advantageous selection in the 1990s 
FIgURE 5.2. Quantile bounds for full sample imposing stochastic dominance 1975-1979 1995-1999
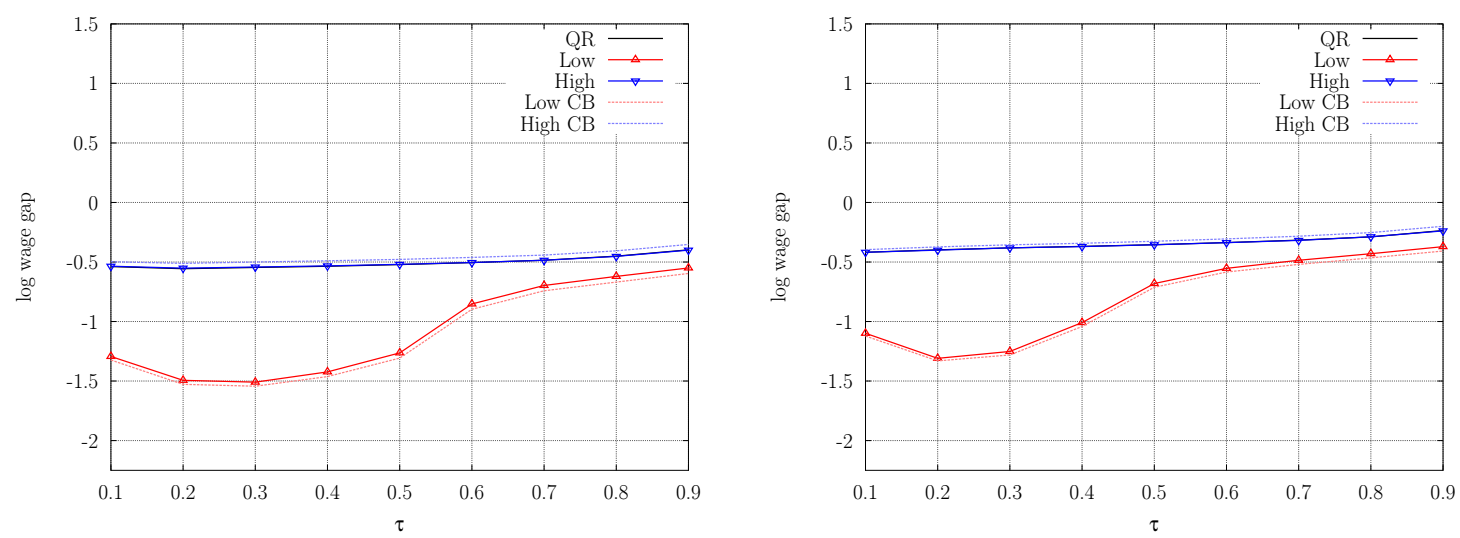

Estimated quantile gender wage (female - male) conditional on average characteristics. The solid black line shows the quantile gender wage when selection is ignored. The blue and red lines with upward and downward pointing triangles show upper and lower bounds that account for employment selection for females. The dashed lines represent a uniform $90 \%$ confidence region for the bounds.

FIGURE 5.3. Quantile bounds for the change in the gender wage gap imposing stochastic dominance

Full sample

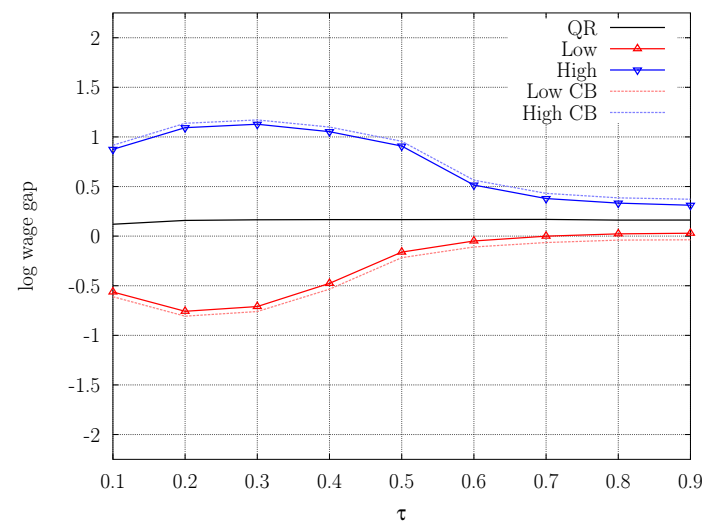

Single and $\geq 16$ years education

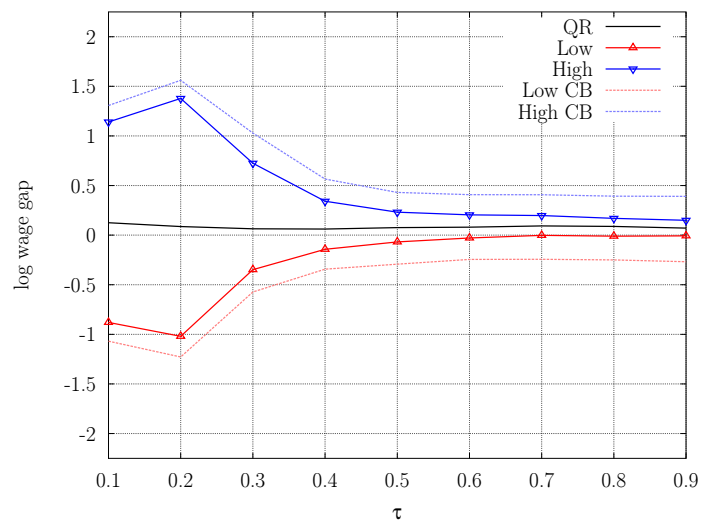

Estimated change (1990s - 1970s) in the quantile gender wage gap (female - male) conditional on having average characteristics. The solid black line shows the quantile gender wage gap when selection is ignored. The blue and red lines with upward and downward pointing triangles show upper and lower bounds that account for employment selection for females. The dashed lines represent a uniform $90 \%$ confidence region for the bounds. 
implies that stochastic dominance did not hold in the 1970s. Accordingly, we also explore some weaker restrictions. Blundell, Gosling, Ichimura, and Meghir (2007) propose a median restriction - that the median wage offer for those not working is less than or equal to the median observed wage. This restriction implies the following bounds on the distribution of wages

$$
\begin{array}{r}
F(y \mid x, u=1) \mathrm{P}(u=1 \mid x)+1\left\{y \geq Q_{y}(0.5 \mid x, u=1)\right\} 0.5 \mathrm{P}(u=0 \mid x) \leq \\
\leq F(y \mid x) \leq F(y \mid x, u=1) \mathrm{P}(u=1 \mid x)+\mathrm{P}(u=0 \mid x),
\end{array}
$$

where $y$ is wage and $u=1$ indicates employment. Transforming these into bounds on the conditional quantiles yields

$$
Q_{0}(\alpha \mid x) \leq Q_{y}(\alpha \mid x) \leq Q_{1}(\alpha \mid x),
$$

where

$$
Q_{0}(\alpha \mid x)= \begin{cases}Q_{y}\left(\frac{\alpha-\mathrm{P}(u=0 \mid x)}{\mathrm{P}(u=1 \mid x)} \mid x, u=1\right) & \text { if } \alpha \geq \mathrm{P}(u=0 \mid x) \\ y_{0} & \text { otherwise }\end{cases}
$$

and

$$
Q_{1}(\alpha \mid x)= \begin{cases}Q_{y}\left(\frac{\alpha}{\mathrm{P}(u=1 \mid x)} \mid x, u=1\right) & \text { if } \alpha<0.5 \& \alpha \leq \mathrm{P}(u=1 \mid x), \\ Q_{y}\left(\frac{\alpha-0.5 \mathrm{P}(u=0 \mid x)}{\mathrm{P}(u=1 \mid x)} \mid x, u=1\right) & \text { if } \alpha \geq 0.5 \& \alpha \leq \frac{1+\mathrm{P}(u=1 \mid x)}{2} \\ y_{1} & \text { otherwise. }\end{cases}
$$

As above, we can also express $Q_{0}(\alpha \mid x)$ and $Q_{1}(\alpha \mid x)$ as the $\alpha$ conditional quantiles of $\tilde{y}_{0}$ and $\tilde{y}_{1}$ where

$$
\tilde{y}_{0}=y 1\{u=1\}+y_{0} 1\{u=0\}
$$

and

$$
\tilde{y}_{1}= \begin{cases}y 1\{u=1\}+y_{1} 1\{u=0\} & \text { with probability } 0.5, \\ y 1\{u=1\}+Q_{y}(0.5 \mid x, u=1) 1\{u=0\} & \text { with probability } 0.5 .\end{cases}
$$

We can easily generalize this median restriction by assuming the $\alpha_{1}$ quantile of wages conditional on working is greater than or equal to the $\alpha_{0}$ quantile of wages conditional on not working. In that case, the bounds can still be expressed as $\alpha$ conditional quantiles of $\tilde{y}_{0}$ and $\tilde{y}_{1}$ with $\tilde{y}_{0}$ as defined above and

$$
\tilde{y}_{1}= \begin{cases}y 1\{u=1\}+y_{1} 1\{u=0\} & \text { with probability }\left(1-\alpha_{0}\right), \\ y 1\{u=1\}+Q_{y}\left(\alpha_{1} \mid x, u=1\right) 1\{u=0\} & \text { with probability } \alpha_{0} .\end{cases}
$$

We can even impose a set of these restrictions for $\left(\alpha_{1}, \alpha_{0}\right) \in \mathcal{R} \subseteq \mathcal{A} \times \mathcal{A}$. Stochastic dominance is equivalent to imposing this restriction for $\alpha_{1}=\alpha_{0}$ for all $\alpha_{1} \in[0,1]$.

Figure 5.4 shows estimates of the gender wage gap with the median restriction for the full sample. Figure C.6 in Appendix C reports the results for the subsample of highly educated singles. The median restriction has substantial identifying power for the presence of a gender wage gap both in the 1970s and in the 1990s, yielding strong evidence of a gender wage gap up to the 0.7 (or slightly higher) quantiles in both the 1970s and 1990s. In comparison, in the absence of this restriction we have strong evidence of a gender wage 
FiguRE 5.4. Quantile bounds for full sample imposing the median restriction

1975-1979

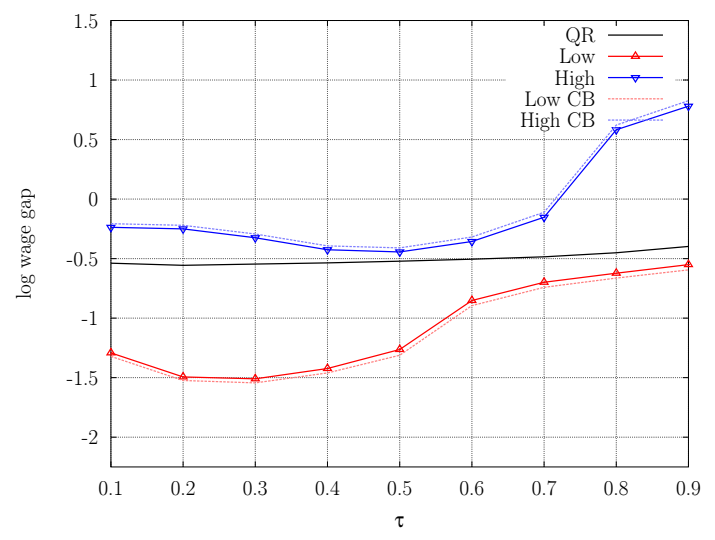

1995-1999

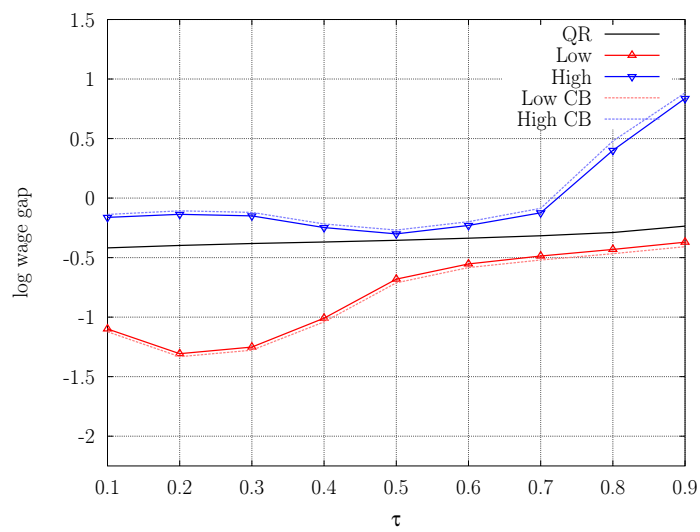

Estimated quantile gender wage (female - male) conditional on average characteristics. The solid black line shows the quantile gender wage when selection is ignored. The blue and red lines with upward and downward pointing triangles show upper and lower bounds that account for employment selection for females. The dashed lines represent a uniform $90 \%$ confidence region for the bounds.

gap only up to the 0.4 quantiles. However, under this weaker restriction there is substantial overlap in the identification regions between the two periods, hence it is not possible to draw strong conclusions about the change in the gender wage gap.

\section{Conclusion}

This paper provides a novel method for inference on best linear approximations to functions which are known to lie within a band. It advances the literature by allowing for bounding functions that may be estimated parametrically or non-parametrically by series estimators, and that may carry an index. Our focus on best linear approximations is motivated by the difficulty to work directly with the sharp identification region of the functions of interest, especially when the analysis is conditioned upon a large number of covariates. By contrast, best linear approximations are tractable and easy to interpret. The sharp identification region of the parameters characterizing the best linear approximation is convex, hence can be equivalently described via its support function. The latter can be estimated with a plug-in method that replaces moments of the data with their sample analogs and the bounding functions with their estimators. We show that the support function process is strongly approximated by a Gaussian process. While this process may not converge weakly as the number of series terms increases to infinity, each subsequence contains a further subsequence that converges weakly to a tight Gaussian process with a uniformly equicontinuous and non-degenerate covariance function. We establish validity of the Bayesian bootstrap for practical inference, and verify our regularity conditions for a large number of empirically relevant problems. 


$$
\begin{aligned}
& \mathcal{S}^{d-1}: \quad=\left\{q \in \mathbb{R}^{d}:\|q\|=1\right\} \\
& T:=\mathcal{S}^{d-1} \times \mathcal{A} \\
& \mathbb{G}_{n}[h(t)]:=\frac{1}{\sqrt{n}} \sum_{i=1}^{n}\left(h_{i}(t)-\mathrm{E} h(t)\right) ; \\
& \mathbb{G}\left[h_{k}\right], \widetilde{\mathbb{G}\left[h_{k}\right]}:=\text { P-Brownian bridge processes, independent of each other, and } \\
& \text { with identical distributions; } \\
& \mathcal{L}^{2}(X, \mathrm{P}) \quad: \quad=\left\{g: X \longrightarrow \mathbb{R} \text { s.t. } \int_{X}|g(x)|^{2} d \mathrm{P}(x)<\infty\right\} \\
& \ell^{\infty}(T) \quad \text { : } \quad \text { set of all uniformly bounded real functions on } T \text {; } \\
& B L_{1}\left(\ell^{\infty}(T),[0,1]\right) \quad \text { : } \quad \text { set of real functions on } \ell^{\infty}(T) \text { with Lipschitz norm bounded by } 1 \text {; } \\
& \lesssim \text { left side bounded by a constant times the right side; } \\
& f^{o}:=f-\mathrm{E} f \text {. }
\end{aligned}
$$

\section{Appendix B. Proof of the Results}

Throughout this Appendix, we impose Conditions C1.C5.

\section{B.1. Proof of Theorems 1 and 2.}

Step 1. We can write the difference between the estimated and true support function as the sum of three differences.

$$
\widehat{\sigma}_{\widehat{\theta}, \widehat{\Sigma}}-\sigma_{\theta, \Sigma}=\left(\widehat{\sigma}_{\widehat{\theta}, \widehat{\Sigma}}-\widehat{\sigma}_{\theta, \widehat{\Sigma}}\right)+\left(\widehat{\sigma}_{\theta, \widehat{\Sigma}}-\widehat{\sigma}_{\theta, \Sigma}\right)+\left(\widehat{\sigma}_{\theta, \Sigma}-\sigma_{\theta, \Sigma}\right)
$$

where $t \in T:=\mathcal{S}^{d-1} \times \mathcal{A}$. Let $\mu:=q^{\prime} \Sigma$ and

$$
w_{i, \mu}(\alpha):=\left(\theta_{0}(x, \alpha) 1\left(\mu z_{i}<0\right)+\theta_{1}(x, \alpha) 1\left(\mu z_{i} \geq 0\right)\right) .
$$

We define

$$
\widehat{\sigma}_{\theta, \widehat{\Sigma}}:=\mathbb{E}_{n}\left[q^{\prime} \widehat{\Sigma} z_{i} w_{i, q^{\prime} \widehat{\Sigma}}(\alpha)\right] \text { and } \widehat{\sigma}_{\theta, \Sigma}:=\mathbb{E}_{n}\left[q^{\prime} \Sigma z_{i} w_{i, q^{\prime} \Sigma}(\alpha)\right] \text {. }
$$

By Lemma 1 uniformly in $t \in T$

$$
\begin{aligned}
\sqrt{n}\left(\widehat{\sigma}_{\widehat{\theta}, \widehat{\Sigma}}-\widehat{\sigma}_{\theta, \widehat{\Sigma}}\right)(t) & =q^{\prime} \Sigma \mathrm{E}\left[z_{i} p_{i}^{\prime} 1\left\{q^{\prime} \Sigma z_{i}>0\right\}\right] J_{1}^{-1}(\alpha) \mathbb{G}_{n}\left[p_{i} \varphi_{i 1}(\alpha)\right] \\
& +q^{\prime} \Sigma \mathrm{E}\left[z_{i} p_{i}^{\prime} 1\left\{q^{\prime} \Sigma z_{i}<0\right\}\right] J_{0}^{-1}(\alpha) \mathbb{G}_{n}\left[p_{i} \varphi_{i 0}(\alpha)\right]+o_{\mathrm{P}}(1) .
\end{aligned}
$$

By Lemma 2 uniformly in $t \in T$

$$
\begin{aligned}
\sqrt{n}\left(\widehat{\sigma}_{\theta, \widehat{\Sigma}}-\widehat{\sigma}_{\theta, \Sigma}\right)(t) & =\sqrt{n} q^{\prime}(\widehat{\Sigma}-\Sigma) \mathrm{E}\left[z_{i} w_{i, q^{\prime} \Sigma}(\alpha)\right]+o_{\mathrm{P}}(1) \\
& =-q^{\prime} \widehat{\Sigma} \mathbb{G}_{n}\left[x_{i} z_{i}^{\prime}\right] \Sigma \mathrm{E}\left[z_{i} w_{i, q^{\prime} \Sigma}(\alpha)\right]+o_{\mathrm{P}}(1) \\
& =-q^{\prime} \Sigma \mathbb{G}_{n}\left[x_{i} z_{i}^{\prime}\right] \Sigma \mathrm{E}\left[z_{i} w_{i, q^{\prime} \Sigma}(\alpha)\right]+o_{\mathrm{P}}(1)
\end{aligned}
$$

By definition

$$
\sqrt{n}\left(\widehat{\sigma}_{\theta, \Sigma}-\sigma_{\theta, \Sigma}\right)(t)=\mathbb{G}_{n}\left[q^{\prime} \Sigma z_{i} w_{i, q^{\prime} \Sigma}(u)\right]
$$


Putting all the terms together uniformly in $t \in T$

$$
\sqrt{n}\left(\widehat{\sigma}_{\widehat{\theta}, \widehat{\Sigma}}-\sigma_{\theta, \Sigma}\right)(t)=\mathbb{G}_{n}\left[h_{k}(t)\right]+o_{\mathrm{P}}(1),
$$

where for $t:=(q, \alpha) \in T=\mathcal{S}^{d-1} \times \mathcal{A}$

$$
\begin{aligned}
h_{k i}(t):= & \quad q^{\prime} \Sigma \mathrm{E}\left[z_{i} p_{i}^{\prime} 1\left\{q^{\prime} \Sigma z_{i}>0\right\}\right] J_{1}^{-1}(\alpha) p_{i} \varphi_{i 1}(\alpha) \\
& +\quad q^{\prime} \Sigma \mathrm{E}\left[z_{i} p_{i}^{\prime} 1\left\{q^{\prime} \Sigma z_{i}<0\right\}\right] J_{0}^{-1}(\alpha) p_{i} \varphi_{i 0}(\alpha) \\
& -\quad q^{\prime} \Sigma x_{i} z_{i}^{\prime} \Sigma \mathrm{E}\left[z_{i} w_{i, q^{\prime} \Sigma}(\alpha)\right] \\
& +\quad q^{\prime} \Sigma z_{i} w_{i, q^{\prime} \Sigma}(\alpha) \\
:= & \quad h_{k 1 i}(t)+h_{k 2 i}(t)+h_{k 3 i}(t)+h_{k 4 i}(t),
\end{aligned}
$$

where $k$ indexes the number of series terms. To simplify notation, we omit the subscript $k$ below.

Step 2. (Finite $k$ ). This case follows from $\mathcal{H}=\left\{h_{i}(t), t \in T\right\}$ being a Donsker class with square-integrable envelopes. Indeed, $\mathcal{H}$ is formed as finite products and sums of $\mathrm{VC}$ classes or entropically equivalent classes, so we can apply Lemma 9. The result

$$
\mathbb{G}_{n}\left[h_{k}(t)\right] \Rightarrow \mathbb{G}\left[h_{k}(t)\right] \text { in } \ell^{\infty}(T),
$$

follows, and the assertion that

$$
\mathbb{G}_{n}\left[h_{k}(t)\right]={ }_{d} \mathbb{G}\left[h_{k}(t)\right]+o_{\mathrm{P}}(1) \text { in } \ell^{\infty}(T)
$$

follows from e.g., the Skorohod-Dudley-Whichura construction. (The $={ }_{d}$ can be replaced by $=$ as in Step 3, in which case $\mathbb{G}\left[h_{k}(t)\right]$ is a sequence of Gaussian processes indexed by $n$ and identically distributed for each $n$.)

Step 3. (Case with growing $k$.) This case is considerably more difficult. The main issue here is that the uniform covering entropy of $\mathcal{H}_{l}=\left\{h_{l i}(t), t \in T\right\}, l=0,1$, grows without bound, albeit at a very slow rate $\log n$. The envelope $H_{l}$ of this class also grows in general, and so we can not rely on the usual uniform entropy-based arguments; for similar reasons we can not rely on the bracketing-based entropy arguments. Instead, we rely on a strong approximation argument, using ideas in Chernozhukov, Lee, and Rosen (2013) and Belloni and Chernozhukov (2009a), to show that $\mathbb{G}_{n}\left[h_{k}(t)\right]$ can be approximated by a tight sequence of Gaussian processes $\mathbb{G}[h(t)]$, implicitly indexed by $k$, where the latter sequence is very well-behaved. Even though it may not converge as $k \rightarrow \infty$, for every subsequence of $k$ there is a further subsequence along which the Gaussian process converges to a well-behaved Gaussian process. The latter is sufficient for carrying out the usual inference.

Lemma 4 below establishes that

$$
\mathbb{G}_{n}[h(t)]=\mathbb{G}[h(t)]+o_{\mathrm{P}}(1) \text { in } \ell^{\infty}(T),
$$

where $\mathbb{G}[h]$ is a sequence of P-Brownian bridges with the covariance function $\mathrm{E}\left[h(t) h\left(t^{\prime}\right)\right]$ $\mathrm{E}[h(t)] \mathrm{E}\left[h\left(t^{\prime}\right)\right]$. Lemma 6 below establishes that for some $0<c \leq 1 / 2$

$$
\rho_{2}\left(h(t), h\left(t^{\prime}\right)\right)=\left(\mathrm{E}\left[h(t)-h\left(t^{\prime}\right)\right]^{2}\right)^{1 / 2} \lesssim \rho\left(t, t^{\prime}\right):=\left\|t-t^{\prime}\right\|^{c},
$$

and the function $\mathrm{E}\left[h(t) h\left(t^{\prime}\right)\right]-\mathrm{E}[h(t)] \mathrm{E}\left[h\left(t^{\prime}\right)\right]$ is equi-continuous on $T \times T$ uniformly in $k$. By assumption C3 we have that $\inf _{t \in T} \operatorname{var}[h(t)]>C>0$, with Lemma 6 providing a sufficient condition for this. 
An immediate consequence of the above result is that we also obtain the convergence in the bounded Lipschitz metric

$$
\sup _{g \in B L_{1}\left(\ell^{\infty}(T),[0,1]\right)}\left|\mathrm{E}\left[g\left(\mathbb{G}_{n}[h]\right)\right]-\mathrm{E}[g(\mathbb{G}[h])]\right| \leq \mathrm{E} \sup _{t \in T}\left|\mathbb{G}_{n}[h(t)]-\mathbb{G}[h(t)]\right| \wedge 1 \rightarrow 0 .
$$

Step 4. Let's recognize the fact that $h$ depends on $k$ by using the notation $h_{k}$ in this step of the proof. Note that $k$ itself is implicitly indexed by $n$. Let $F_{k}(c):=\mathrm{P}\left\{f\left(\mathbb{G}\left[h_{k}\right]\right) \leq c\right\}$ and observe that by Step 3 and $f \in \mathcal{F}_{c}$

$$
\begin{aligned}
& \left|\mathrm{P}\left\{f\left(S_{n}\right) \leq c_{n}+o_{p}(1)\right\}-\mathrm{P}\left\{f\left(\mathbb{G}\left[h_{k}\right]\right) \leq c_{n}\right\}\right| \\
\leq & \left|\mathrm{P}\left\{f\left(\mathbb{G}\left[h_{k}\right]\right) \leq c_{n}+o_{p}(1)\right\}-\mathrm{P}\left\{f\left(\mathbb{G}\left[h_{k}\right]\right) \leq c_{n}\right\}\right| \\
\leq & \delta_{n}\left(o_{p}(1)\right) \rightarrow_{P} 0, \quad \text { for } \delta_{n}(\epsilon):=\sup _{c \in \mathbb{R}}\left|F_{k}(c+\epsilon)-F_{k}(c)\right|,
\end{aligned}
$$

where the last step follows by the Extended Continuous Mapping Theorem (Theorem 18.11 in van der Vaart (2000)) provided that we can show that for any $\epsilon_{n} \searrow 0, \delta_{n}\left(\epsilon_{n}\right) \rightarrow 0$. Suppose otherwise, then there is a subsequence along which $\delta_{n}\left(\epsilon_{n}\right) \rightarrow \delta \neq 0$. We can select a further subsequence say $\left\{n_{j}\right\}$ along which the covariance function of $\mathbb{G}_{n}\left[h_{k}\right]$, denoted $\Omega_{n k}\left(t, t^{\prime}\right)$ converges to a covariance function $\Omega_{0}\left(t, t^{\prime}\right)$ uniformly on $T \times T$. We can do so by the ArzelàAscoli theorem in view of the uniform equicontinuity in $k$ of the sequence of the covariance functions $\Omega_{n k}\left(t, t^{\prime}\right)$ on $T \times T$. Moreover, $\inf _{t \in T} \Omega_{0}(t, t)>C>0$ by our assumption on $\Omega_{n k}\left(t, t^{\prime}\right)$. But along this subsequence $\mathbb{G}\left[h_{k}\right]$ converges in $\ell^{\infty}(T)$ in probability to a tight Gaussian process, say $Z_{0}$. The latter happens because $\mathbb{G}\left[h_{k}\right]$ converges to $Z_{0}$ marginally by Gaussianity and by $\Omega_{n k}\left(t, t^{\prime}\right) \rightarrow \Omega_{0}\left(t, t^{\prime}\right)$ uniformly and hence pointwise on $T \times T$ and because $\mathbb{G}\left[h_{k}\right]$ is asymptotically equicontinuous as shown in the proof of Lemma 4 . Thus, along this subsequence we have that

$$
F_{k}(c) \rightarrow F_{0}(c)=\mathrm{P}\left\{f\left(Z_{0}\right) \leq c\right\}, \text { uniformly in } c \in \mathbb{R},
$$

because we have pointwise convergence that implies uniform convergence by Polya's theorem, since $F_{0}$ is continuous by $f \in \mathcal{F}_{c}$ and by $\inf _{t \in T} \Omega_{0}(t, t)>C>0$. This implies that along this subsequence $\delta_{n_{j}}\left(\epsilon_{n_{j}}\right) \rightarrow 0$, which gives a contradiction.

Step 5. Finally, we observe that $c(1-\tau)=O(1)$ holds by $\sup _{t \in T}\left\|\mathbb{G}\left[h_{k}(t)\right]\right\|=O_{\mathrm{P}}(1)$ as shown in the proof of Lemma 4, and the second part of Theorem 2 follows.

\section{B.2. Proof of Theorems 3 and 4.}

Step 1. We can write the difference between a bootstrap and true support function as the sum of three differences.

$$
\widetilde{\sigma}_{\widetilde{\theta}, \widetilde{\Sigma}}-\sigma_{\theta, \Sigma}=\left(\widetilde{\sigma}_{\widetilde{\theta}, \widetilde{\Sigma}}-\widetilde{\sigma}_{\theta, \widetilde{\Sigma}}\right)+\left(\widetilde{\sigma}_{\theta, \widetilde{\Sigma}}-\widetilde{\sigma}_{\theta, \Sigma}\right)+\left(\widetilde{\sigma}_{\theta, \Sigma}-\sigma_{\theta, \Sigma}\right)
$$

where for

$$
w_{i, \mu}(\alpha)=:\left(\theta_{0}(x, \alpha) 1\left(\mu z_{i}<0\right)+\theta_{1}(x, \alpha) 1\left(\mu z_{i} \geq 0\right)\right)
$$

we define

$$
\widetilde{\sigma}_{\theta, \widetilde{\Sigma}}:=\mathbb{E}_{n}\left[\left(e_{i} / \bar{e}\right) q^{\prime} \widetilde{\Sigma}^{\prime} z_{i} w_{i, q^{\prime} \widetilde{\Sigma}}(\alpha)\right] \text { and } \widetilde{\sigma}_{\theta, \Sigma}=: \mathbb{E}_{n}\left[\left(e_{i} / \bar{e}\right) q^{\prime} \Sigma^{\prime} z_{i} w_{i, q^{\prime} \Sigma}(\alpha)\right],
$$

where $\bar{e}=\mathbb{E}_{n} e_{i} \rightarrow_{\mathrm{P}} 1$. 
By Lemma 1 uniformly in $t \in T$

$$
\begin{aligned}
\sqrt{n}\left(\widetilde{\sigma}_{\widetilde{\theta}, \widetilde{\Sigma}}-\widetilde{\sigma}_{\theta, \widetilde{\Sigma}}\right)(t) & =q^{\prime} \Sigma \mathrm{E}\left[z_{i} p_{i}^{\prime} 1\left\{q^{\prime} \Sigma z_{i}>0\right\}\right] J_{1}^{-1}(\alpha) \mathbb{G}_{n}\left[e_{i} p_{i} \varphi_{i 1}(\alpha)\right] \\
& +q^{\prime} \Sigma \mathrm{E}\left[z_{i} p_{i}^{\prime} 1\left\{q^{\prime} \Sigma z_{i}<0\right\}\right] J_{0}^{-1}(\alpha) \mathbb{G}_{n}\left[e_{i} p_{i} \varphi_{i 0}(\alpha)\right]+o_{\mathrm{P}}(1) .
\end{aligned}
$$

By Lemma 2 uniformly in $t \in T$

$$
\begin{aligned}
\sqrt{n}\left(\widetilde{\sigma}_{\theta, \widetilde{\Sigma}}-\widetilde{\sigma}_{\theta, \Sigma}\right)(t) & =\sqrt{n} q^{\prime}(\widetilde{\Sigma}-\Sigma) \mathrm{E}\left[z_{i} w_{i, q^{\prime} \Sigma}(\alpha)\right]+o_{\mathrm{P}}(1) \\
& =q^{\prime} \widetilde{\Sigma} \mathbb{G}_{n}\left[\left(e_{i} / \bar{e}\right)\left(x_{i} z_{i}^{\prime}\right)^{o}\right] \Sigma \mathrm{E}\left[z_{i} w_{i, q^{\prime} \Sigma}(\alpha)\right]+o_{\mathrm{P}}(1) \\
& =q^{\prime} \Sigma \mathbb{G}_{n}\left[e_{i}\left(x_{i} z_{i}^{\prime}\right)^{o}\right] \Sigma \mathrm{E}\left[z_{i} w_{i, q^{\prime} \Sigma}(\alpha)\right]+o o_{\mathrm{P}}(1) .
\end{aligned}
$$

By definition

$$
\sqrt{n}\left(\widetilde{\sigma}_{\theta, \Sigma}-\sigma_{\theta, \Sigma}\right)(t)=\mathbb{G}_{n}\left[e_{i}\left(q^{\prime} \Sigma z_{i} w_{i, q^{\prime} \Sigma}(\alpha)\right)^{o}\right] / \bar{e}=\mathbb{G}_{n}\left[e_{i}\left(q^{\prime} \Sigma z_{i} w_{i, q^{\prime} \Sigma}(\alpha)\right)^{o}\right]\left(1+o_{p}(1)\right) .
$$

Putting all the terms together uniformly in $t \in T$

$$
\sqrt{n}\left(\widetilde{\sigma}_{\widetilde{\theta}, \widetilde{\Sigma}}-\sigma_{\theta, \Sigma}\right)(t)=\mathbb{G}_{n}\left[e_{i} h_{i}^{o}(t)\right]+o_{\mathrm{P}}(1) .
$$

Step 2. Combining conclusions of Theorems 1 and Step 1 above we obtain:

$$
\begin{aligned}
& \tilde{S}_{n}(t)=\sqrt{n}\left(\widetilde{\sigma}_{\widetilde{\theta}, \widetilde{\Sigma}}-\widehat{\sigma}_{\widehat{\theta}, \widehat{\Sigma}}\right)(t) \\
= & \sqrt{n}\left(\widetilde{\sigma}_{\widetilde{\theta}, \widetilde{\Sigma}}-\sigma_{\theta, \Sigma}\right)(t)-\sqrt{n}\left(\widehat{\sigma}_{\widehat{\theta}, \widehat{\Sigma}}-\sigma_{\theta, \Sigma}\right)(t) \\
= & \mathbb{G}_{n}\left[e_{i} h_{i}^{o}(t)\right]-\mathbb{G}_{n}[h(t)]+o_{\mathrm{P}}(1) \\
= & \mathbb{G}_{n}\left[e_{i}^{o} h_{i}^{o}(t)\right]+o_{\mathrm{P}}(1) .
\end{aligned}
$$

Observe that the bootstrap process $\mathbb{G}_{n}\left[e_{i}^{o} h_{i}^{o}(t)\right]$ has the unconditional covariance function

$$
\mathrm{E}\left[h(t) h\left(t^{\prime}\right)\right]-\mathrm{E}[h(t)] \mathrm{E}\left[h\left(t^{\prime}\right)\right],
$$

which is equal to the covariance function of the original process $\mathbb{G}_{n}\left[h_{i}\right]$. Conditional on data the covariance function of this process is

$$
\mathbb{E}_{n}\left[h(t) h\left(t^{\prime}\right)\right]-\mathbb{E}_{n}[h(t)] \mathbb{E}_{n}\left[h\left(t^{\prime}\right)\right] .
$$

Comment B.1. Note that if a bootstrap random element $Z_{n}$ taking values in a normed space $(E,\|\cdot\|)$ converges in probability $\mathrm{P}$ unconditionally, that is $Z_{n}=o_{\mathrm{P}}(1)$, then $Z_{n}=$ $o_{\mathrm{P} e}(1)$ in $L^{1}(P)$ sense and hence probability $\mathrm{P}$, where $\mathrm{P}^{e}$ denotes the probability measure conditional on the data. In other words, $Z_{n}$ also converges in probability conditionally on the data. This follows because $\mathrm{E}_{\mathrm{P}}\left|\mathrm{P}^{e}\left\{\left\|Z_{n}\right\|>\epsilon\right\}\right|=\mathrm{P}\left\{\left\|Z_{n}\right\|>\epsilon\right\} \rightarrow 0$, so that $\mathrm{P}^{e}\left\{\left\|Z_{n}\right\|>\epsilon\right\} \rightarrow$ 0 in $L^{1}(P)$ sense and hence in probability P. Similarly, if $Z_{n}=O_{\mathrm{P}}(1)$, then $Z_{n}=O_{\mathrm{P} e}(1)$ in probability $\mathrm{P}$.

Step 3. (Finite $k$ ). This case follows from $\mathcal{H}=\left\{h_{i}(t), t \in T\right\}$ being a Donsker class with square-integrable envelopes. Indeed, $\mathcal{H}$ is formed as a Lipschitz composition of VC classes or entropically equivalent classes. Then by the Donsker theorem for exchangeable bootstraps, see e.g., van der Vaart and Wellner (1996), we have weak convergence conditional on the data

$$
\mathbb{G}_{n}\left[e_{i}^{o} h_{i}^{o}(t)\right] / \bar{e} \Rightarrow \widetilde{\mathbb{G}[h(t)]} \text { under } \mathrm{P}^{e} \text { in } \ell^{\infty}(T) \text { in probability } \mathrm{P},
$$


where $\widetilde{\mathbb{G}[h]}$ is a sequence of P-Brownian bridges independent of $\mathbb{G}[h]$ and with the same distribution as $\mathbb{G}[h]$. In particular, the covariance function of $\widetilde{\mathbb{G}[h}]$ is $\mathrm{E}\left[h(t) h\left(t^{\prime}\right)\right]-\mathrm{E}[h(t)] \mathrm{E}\left[h\left(t^{\prime}\right)\right]$. Since $\bar{e} \rightarrow$ pe 1 , the above implies

$$
\left.\mathbb{G}_{n}\left[e_{i}^{o} h_{i}^{o}(t)\right] \Rightarrow \widetilde{\mathbb{G}[h(t)}\right] \text { under } \mathrm{P}^{e} \text { in } \ell^{\infty}(T) \text { in probability P. }
$$

The latter statement simply means

$$
\left.\sup _{g \in B L_{1}\left(\ell^{\infty}(T),[0,1]\right)} \mid \mathrm{E}_{\mathrm{P} e}\left[g\left(\mathbb{G}_{n}[h]\right)\right]-\mathrm{E}[g(\widetilde{\mathbb{G}[h}])\right] \mid \rightarrow_{\mathrm{P}} 0 .
$$

This statement can be strengthened to a coupling statement as in Step 4 .

Step 4. (Growing $k$.) By Lemma 4 below we can show that (on a suitably extended probability space) there exists a sequence of Gaussian processes $\widetilde{\mathbb{G}[h(t)]}$ such that

$$
\mathbb{G}_{n}\left[e_{i}^{o} h_{i}^{o}(t)\right]=\widetilde{\mathbb{G}[h(t)]}+o_{\mathrm{P}}(1) \text { in } \ell^{\infty}(T),
$$

which implies by Remark B.1 that

$$
\mathbb{G}_{n}\left[e_{i}^{o} h_{i}^{o}(t)\right]=\widetilde{\mathbb{G}[h(t)]}+o_{\mathrm{P} e}(1) \text { in } \ell^{\infty}(T) \text { in probability. }
$$

Here, as above, $\widetilde{\mathbb{G}[h]}$ is a sequence of P-Brownian bridges independent of $\mathbb{G}[h]$ and with the same distribution as $\mathbb{G}[h]$. In particular, the covariance function of $\widetilde{\mathbb{G}[h]}$ is $\mathrm{E}\left[h(t) h\left(t^{\prime}\right)\right]$ $\mathrm{E}[h(t)] \mathrm{E}\left[h\left(t^{\prime}\right)\right]$. Lemma 6 describes the properties of this covariance function, which in turn define the properties of this Gaussian process.

An immediate consequence of the above result is the convergence in bounded Lipschitz metric

$\sup _{g \in B L_{1}\left(\ell^{\infty}(T),[0,1]\right)}\left|\mathrm{E}_{\mathrm{P} e}\left[g\left(\mathbb{G}_{n}\left[e_{i}^{o} h_{i}^{o}(t)\right]\right)\right]-\mathrm{E}_{\mathrm{P} e}[g(\widetilde{\mathbb{G}[h]})]\right| \leq \mathrm{E}_{\mathrm{P} e} \sup _{t \in T}\left|\mathbb{G}_{n}\left[e_{i}^{o} h_{i}^{o}(t)\right]-\widetilde{\mathbb{G}[h(t)]}\right| \wedge 1 \rightarrow_{\mathrm{P}} 0$.

Note that $\mathrm{E}_{\mathrm{P} e}[g(\widetilde{\mathbb{G}[h]})]=\mathrm{E}_{\mathrm{P}}[g(\widetilde{\mathbb{G}[h]})]$, since the covariance function of $\left.\widetilde{\mathbb{G}[h}\right]$ does not depend on the data. Therefore

$$
\sup _{g \in B L_{1}\left(\ell^{\infty}(T),[0,1]\right)}\left|\mathrm{E}_{\mathrm{P} e}\left[g\left(\mathbb{G}_{n}\left[e_{i}^{o} h_{i}^{o}(t)\right]\right)\right]-\mathrm{E}_{\mathrm{P}}[g(\widetilde{\mathbb{G}[h]})]\right| \rightarrow_{\mathrm{P}} 0 .
$$

Step 5. Let us recognize the fact that $h$ depends on $k$ by using the notation $h_{k}$ in this step of the proof. Note that $k$ itself is implicitly indexed by $n$. By the previous steps and Theorem 1 there exist $\epsilon_{n} \searrow 0$ such that $\pi_{1}=\mathrm{P}^{e}\left\{\left|f\left(\widetilde{S}_{n}\right)-f\left(\widetilde{\mathbb{G}\left[h_{k}\right]}\right)\right|>\epsilon_{n}\right\}$ and $\pi_{2}=\mathrm{P}\left\{\left|f\left(\widetilde{S}_{n}\right)-f\left(\mathbb{G}\left[h_{k}\right]\right)\right|>\epsilon_{n}\right\}$ obey $\mathrm{E}\left[\pi_{1}\right] \rightarrow_{P} 0$ and $\pi_{2} \rightarrow 0$. Let

$$
F(c):=\mathrm{P}\left\{f\left(\mathbb{G}\left[h_{k}\right]\right) \leq c\right\}=\mathrm{P}\left\{f\left(\widetilde{\mathbb{G}\left[h_{k}\right]}\right) \leq c\right\}=\mathrm{P}^{e}\left\{f\left(\mathbb{G}\left[h_{k}\right]\right) \leq c\right\},
$$

where the equality holds because $\mathbb{G}\left[h_{k}\right]$ and $\widetilde{\mathbb{G}\left[h_{k}\right]}$ are P-Brownian bridges with the same covariance kernel, which in the case of the bootstrap does not depend on the data. 
For any $c_{n}$ which is a measurable function of the data,

$$
\begin{aligned}
& \mathrm{E}\left|\mathrm{P}^{e}\left\{f\left(\widetilde{S}_{n}\right) \leq c_{n}\right\}-\mathrm{P}\left\{f\left(S_{n}\right) \leq c_{n}\right\}\right| \\
\leq & \mathrm{E}\left[\mathrm{P}^{e}\left\{f\left(\widetilde{\mathbb{G}\left[h_{k}\right]}\right) \leq c_{n}+\epsilon_{n}\right\}-\mathrm{P}\left\{f\left(\mathbb{G}\left[h_{k}\right]\right) \leq c_{n}-\epsilon_{n}\right\}+\pi_{1}+\pi_{2}\right] \\
= & \operatorname{E} F\left(c_{n}+\epsilon_{n}\right)-\mathrm{E} F\left(c_{n}-\epsilon_{n}\right)+o(1) \\
\leq & \sup _{c \in \mathbb{R}}\left|F\left(c+\epsilon_{n}\right)-F\left(c-\epsilon_{n}\right)\right|+o(1)=o(1),
\end{aligned}
$$

where the last step follows from the proof of Theorem 1. This proves the first claim of Theorem 4 by the Chebyshev inequality. The second claim of Theorem 4 follows similarly to Step 5 in the proof of Theorems 1-2.

\section{B.3. Main Lemmas for the Proofs of Theorems 1 and 2.}

Lemma 1 (Linearization). 1. (Sample) We have that uniformly in $t \in T$

$$
\begin{aligned}
\sqrt{n}\left(\widehat{\sigma}_{\widehat{\theta}, \widehat{\Sigma}}-\widehat{\sigma}_{\theta, \widehat{\Sigma}}\right) & =q^{\prime} \widehat{\Sigma} \sqrt{n}\left(\mathbb{E}_{n} z_{i}\left(\widehat{\theta}_{1, i}(\alpha)-\theta_{1, i}(\alpha)\right) 1\left\{q^{\prime} \widehat{\Sigma} z_{i}>0\right\}\right) \\
& +q^{\prime} \widehat{\Sigma} \sqrt{n}\left(\mathbb{E}_{n} z_{i}\left(\widehat{\theta}_{0, i}(\alpha)-\theta_{0, i}(\alpha)\right) 1\left\{q^{\prime} \widehat{\Sigma} z_{i}<0\right\}\right) \\
& =q^{\prime} \Sigma \mathrm{E}\left[z_{i} p_{i}^{\prime} 1\left\{q^{\prime} \Sigma z_{i}>0\right\}\right] J_{1}^{-1}(\alpha) \mathbb{G}_{n}\left[p_{i} \varphi_{i 1}(\alpha)\right] \\
& +q^{\prime} \Sigma \mathrm{E}\left[z_{i} p_{i}^{\prime} 1\left\{q^{\prime} \Sigma z_{i}<0\right\}\right] J_{0}^{-1}(\alpha) \mathbb{G}_{n}\left[p_{i} \varphi_{i 0}(\alpha)\right]+o_{\mathrm{P}}(1) .
\end{aligned}
$$

2. (Bootstrap) We have that uniformly in $t \in T$

$$
\begin{aligned}
\sqrt{n}\left(\widetilde{\sigma}_{\widetilde{\theta}, \widetilde{\Sigma}}-\widetilde{\sigma}_{\theta, \widetilde{\Sigma}}\right)(t) & =q^{\prime} \widetilde{\Sigma} \sqrt{n}\left(\mathbb{E}_{n}\left(e_{i} / \bar{e}\right) z_{i}\left(\widetilde{\theta}_{1, i}(\alpha)-\theta_{1, i}(\alpha)\right) 1\left\{q^{\prime} \widetilde{\Sigma} z_{i}>0\right\}\right) \\
& +q^{\prime} \widetilde{\Sigma} \sqrt{n}\left(\mathbb{E}_{n}\left(e_{i} / \bar{e}\right) z_{i}\left(\widetilde{\theta}_{0, i}(\alpha)-\theta_{0, i}(\alpha)\right) 1\left\{q^{\prime} \widetilde{\Sigma} z_{i}<0\right\}\right) \\
& =q^{\prime} \Sigma \mathrm{E}\left[z_{i} p_{i}^{\prime} 1\left\{q^{\prime} \Sigma z_{i}>0\right\}\right] J_{1}^{-1}(\alpha) \mathbb{G}_{n}\left[e_{i} p_{i} \varphi_{i 1}(\alpha)\right] \\
& +q^{\prime} \Sigma \mathrm{E}\left[z_{i} p_{i}^{\prime} 1\left\{q^{\prime} \Sigma z_{i}<0\right\}\right] J_{0}^{-1}(\alpha) \mathbb{G}_{n}\left[e_{i} p_{i} \varphi_{i 0}(\alpha)\right]+o_{\mathrm{P}}(1) .
\end{aligned}
$$

Proof of Lemma 1. In order to cover both cases with one proof, we will use $\bar{\theta}$ to mean either the unweighted estimator $\widehat{\theta}$ or the weighted estimator $\tilde{\theta}$, and $v_{i}$ to mean either 1 in the case of the unweighted estimator or exponential weights $e_{i}$ in the case of the weighted estimator. We also observe that $\bar{\Sigma} \rightarrow_{P} \Sigma$ by the law of large numbers and the continuous mapping theorem.

Step 1. It will suffice to show that

$$
\begin{aligned}
& q^{\prime} \bar{\Sigma} \sqrt{n}\left(\mathbb{E}_{n}\left(v_{i} / \bar{v}\right) z_{i}\left(\bar{\theta}_{1, i}(\alpha)-\theta_{1, i}(\alpha)\right) 1\left\{q^{\prime} \bar{\Sigma} z_{i}>0\right\}\right) \\
= & q^{\prime} \Sigma \mathrm{E}\left[z_{i} p_{i}^{\prime} 1\left\{q \Sigma z_{i}>0\right\}\right] J_{1}^{-1}(\alpha) \mathbb{G}_{n}\left[v_{i} p_{i} \varphi_{i 1}(\alpha)\right]+o_{\mathrm{P}}(1)
\end{aligned}
$$

and that

$$
\begin{aligned}
& q^{\prime} \bar{\Sigma} \sqrt{n}\left(\mathbb{E}_{n}\left(v_{i} / \bar{v}\right) z_{i}\left(\bar{\theta}_{0, i}(\alpha)-\theta_{0, i}(\alpha)\right) 1\left\{q^{\prime} \bar{\Sigma} z_{i}<0\right\}\right) \\
= & q^{\prime} \Sigma \mathrm{E}\left[z_{i} p_{i}^{\prime} 1\left\{q \Sigma z_{i}<0\right\}\right] J_{0}^{-1}(\alpha) \mathbb{G}_{n}\left[v_{i} p_{i} \varphi_{i 0}(\alpha)\right]+o_{\mathrm{P}}(1) .
\end{aligned}
$$


We show the argument for the first part; the argument for the second part is identical. We also drop the index $\ell=1$ to ease notation. By Assumption C2 we write

$$
\begin{aligned}
q^{\prime} \bar{\Sigma} \sqrt{n} \mathbb{E}_{n}\left(v_{i} / \bar{v}\right) z_{i}\left(\bar{\theta}_{i}-\theta_{i}\right) 1\left\{q^{\prime} \bar{\Sigma} z_{i}>0\right\}= & \left\{q ^ { \prime } \overline { \Sigma } \mathbb { E } _ { n } \left[\left(v_{i} z_{i} p_{i}^{\prime} 1\left\{q^{\prime} \bar{\Sigma} z_{i}>0\right\}\right] J^{-1}(\alpha) \mathbb{G}_{n}\left[v_{i} p \varphi_{i}(\alpha)\right]\right.\right. \\
& \left.+q^{\prime} \bar{\Sigma} \mathbb{E}_{n}\left[v_{i} z_{i} \bar{R}_{i}(\alpha) 1\left\{q^{\prime} \bar{\Sigma} z_{i}>0\right\}\right]\right\} / \bar{v} \\
= & :(a(\alpha)+b(\alpha)) /\left(1+o_{\mathrm{P}}(1)\right) .
\end{aligned}
$$

We have from the assumptions of the theorem

$$
\sup _{\alpha \in \mathcal{A}}|b(\alpha)| \leq\left\|q^{\prime} \bar{\Sigma}\right\| \cdot\|\| z_{i}\|\|_{\mathbb{P}_{n}, 2}\|\| v_{i}\|\|_{\mathbb{P}_{n}, 2} \cdot \sup _{\alpha \in \mathcal{A}}\left\|\bar{R}_{i}(\alpha)\right\|_{\mathbb{P}_{n}, 2}=O_{\mathrm{P}}(1) O_{\mathrm{P}}(1) o_{\mathrm{P}}(1)=o_{\mathrm{P}}(1) .
$$

Write $a(\alpha)=c(\alpha)+d(\alpha)$, where

$$
\begin{array}{rlrl}
c(\alpha) & := & & q^{\prime} \Sigma \mathrm{E}\left[z_{i} p_{i}^{\prime} 1\left\{q \sum z_{i}>0\right\}\right] J^{-1}(\alpha) \mathbb{G}_{n}\left[v_{i} p_{i} \varphi_{i}(\alpha)\right] \\
d(\alpha):= & \bar{\mu}^{\prime} J^{-1}(\alpha) \mathbb{G}_{n}\left[v_{i} p_{i} \varphi_{i}(\alpha)\right] \\
\bar{\mu}^{\prime}:= & q^{\prime} \bar{\Sigma} \mathbb{E}_{n}\left[v_{i} z_{i} p_{i}^{\prime} 1\left\{q^{\prime} \bar{\Sigma} z_{i}>0\right\}\right]-q^{\prime} \Sigma \mathrm{E}\left[z_{i} p_{i}^{\prime} 1\left\{q^{\prime} \Sigma z_{i}>0\right\}\right]
\end{array}
$$

The claim follows after showing that $\sup _{\alpha \in \mathcal{A}}|d(\alpha)|=o_{\mathrm{P}}(1)$, which is shown in subsequent steps below.

Step 2. (Special case, with $k$ fixed). This is the parametric case, which is trivial. In this step we have to show $\sup _{\alpha \in \mathcal{A}}|d(\alpha)|=o_{\mathrm{P}}(1)$. We can write

$$
d(\alpha)=\mathbb{G}_{n}\left[\bar{\mu}^{\prime} f_{\alpha}\right], \quad f_{\alpha}:=\left(f_{\alpha j}, j=1, \ldots, k\right), f_{\alpha j}:=J^{-1}(\alpha) v_{i} p_{i j} \varphi_{i}(\alpha)
$$

and define the function class $\mathcal{F}:=\left\{f_{\alpha j}, \alpha \in \mathcal{A}, j=1, \ldots, k\right\}$. Since $k$ is finite, and given the assumptions on $\mathcal{F}_{1}=\{\varphi(\alpha), \alpha \in \mathcal{A}\}$, application of Lemmas 9 and $10-2$ (a) yields

$$
\sup _{Q} \log N\left(\epsilon\|F\|_{Q, 2}, \mathcal{F}, L_{2}(Q)\right) \lesssim \log (1 / \epsilon) .
$$

and the envelope is $\mathrm{P}$-square integrable. Therefore, $\mathcal{F}$ is $\mathrm{P}$-Donsker and

$$
\sup _{\alpha \in \mathcal{A}}\left|\mathbb{G}_{n}\left[f_{\alpha}\right]\right| \lesssim_{\mathrm{P}} 1
$$

and $\sup _{\alpha \in \mathcal{A}}|d(\alpha)| \lesssim{ }_{\mathrm{P}} k\|\bar{\mu}\| \rightarrow_{\mathrm{P}} 0$.

Step 3. (General case, with $k \rightarrow \infty$ ). In this step we have to show $\sup _{\alpha \in \mathcal{A}}|d(\alpha)|=o_{\mathrm{P}}(1)$. The case of $k \rightarrow \infty$ is much more difficult if we want to impose rather weak conditions on the number of series terms. We can write

$$
d(\alpha)=\mathbb{G}_{n}\left[f_{\alpha n}\right], \quad f_{\alpha n}:=\bar{\mu}^{\prime} J^{-1}(\alpha) v_{i} p_{i} \varphi_{i}(\alpha)
$$

and define the function class $\mathcal{F}_{3}:=\left\{f_{\alpha n}, \alpha \in \mathcal{A}\right\}$, see equation B.11 below. By Lemma 10 the random entropy of this function class obeys

$$
\log N\left(\epsilon\left\|F_{3}\right\|_{\mathbb{P}_{n}, 2}, \mathcal{F}_{3}, L_{2}\left(\mathbb{P}_{n}\right)\right) \lesssim_{\mathrm{P}} \log n+\log (1 / \epsilon) .
$$

Therefore by Lemma 12 , conditional on $X_{n}=\left(x_{i}, z_{i}, i=1, \ldots, n\right)$, for each $\delta>0$ there exists a constant $K_{\delta}$, that does not depend on $n$, such that for all $n$ :

$$
\mathrm{P}\left\{\sup _{\alpha \in \mathcal{A}}|d(\alpha)| \geq K_{\delta} \sqrt{\log n}\left(\sup _{\alpha \in \mathcal{A}}\left\|f_{\alpha n}\right\|_{\mathbb{P}_{n}, 2} \vee \sup _{\alpha \in \mathcal{A}}\left\|f_{\alpha n}\right\|_{\mathrm{P} \mid X_{n}, 2}\right)\right\} \leq \delta,
$$

where $\mathrm{P} \mid X_{n}$ denotes the probability measure conditional on $X_{n}$. The conclusion follows if we can demonstrate that $\sqrt{\log n} \sup _{\alpha \in \mathcal{A}}\left\|f_{\alpha n}\right\|_{\mathbb{P}_{n}, 2} \vee \sup _{\alpha \in \mathcal{A}}\left\|f_{\alpha n}\right\|_{\mathrm{P} \mid X_{n}, 2} \rightarrow_{\mathrm{P}} 0$. Note that 


$$
\sup _{\alpha \in \mathcal{A}}\left\|f_{\alpha n}\right\|_{\mathbb{P}_{n}, 2} \leq\|\bar{\mu}\| \sup _{\alpha \in \mathcal{A}}\left\|J^{-1}(\alpha)\right\|\left\|\mathbb{E}_{n} p_{i} p_{i}^{\prime}\right\| \cdot \sup _{i \leq n} v_{i} \sup _{i \leq n, \alpha \in \mathcal{A}}\left|\varphi_{i}(\alpha)\right| \rightarrow_{\mathrm{P}} 0,
$$

where the convergence to zero in probability follows because

$$
\|\bar{\mu}\| \lesssim_{\mathrm{P}} n^{-m / 4}+\sqrt{(k / n) \cdot \log n} \cdot\left(\log n \max _{i}\left\|z_{i}\right\|\right) \wedge \xi_{k}, \quad \sup _{i \leq n} v_{i} \lesssim_{\mathrm{P}} \log n
$$

by Step 4 below, $\sup _{\alpha \in \mathcal{A}}\left\|J^{-1}(\alpha)\right\| \lesssim 1$ by assumption $\mathrm{C} 3$. $\left\|\mathbb{E}_{n} p_{i} p_{i}^{\prime}\right\| \lesssim_{\mathrm{P}} 1$ by Lemma 11 . and

$$
\log ^{2} n\left(n^{-m / 4}+\sqrt{(k / n) \cdot \log n} \cdot \max _{i}\left\|z_{i}\right\| \wedge \xi_{k}\right) \sup _{i \leq n, \alpha \in \mathcal{A}}\left|\varphi_{i}(\alpha)\right| \rightarrow_{\mathrm{P}} 0
$$

by assumption C4. Also note that

$$
\sup _{\alpha \in \mathcal{A}}\left\|f_{\alpha n}\right\|_{\mathrm{P} \mid X_{n}, 2} \leq\|\bar{\mu}\| \sup _{\alpha \in \mathcal{A}}\left\|J^{-1}(\alpha)\right\|\left\|\mathbb{E}_{n} p_{i} p_{i}^{\prime}\right\| \cdot\left(\mathrm{E}\left[v_{i}^{2}\right]\right)^{1 / 2} \cdot \sup _{i \leq n, \alpha \in \mathcal{A}}\left[\mathrm{E}\left[\varphi_{i}^{2}(u) \mid x_{i}, z_{i}\right]\right]^{1 / 2} \rightarrow_{\mathrm{P}} 0
$$

by the preceding argument and $\mathrm{E}\left[\varphi_{i}^{2}(u) \mid x_{i}, z_{i}\right]$ uniformly bounded in $\alpha$ and $i$ by assumption C3.

Step 4. In this step we show that

$$
\|\bar{\mu}\| \lesssim_{\mathrm{P}} n^{-m / 4}+\sqrt{(k / n) \cdot \log n} \cdot\left(\log n \max _{i}\left\|z_{i}\right\|\right) \wedge \xi_{k}
$$

We can bound

$$
\|\bar{\mu}\| \leq\|\Sigma-\bar{\Sigma}\|\left\|\mathrm{E}\left[z_{i} p_{i} 1\left\{q^{\prime} \Sigma z_{i}>0\right\}\right]\right\|+\|\bar{\Sigma}\| \mu_{1}+\|\bar{\Sigma}\| \mu_{2}
$$

where

$$
\begin{aligned}
& \mu_{1}=\left\|\mathbb{E}_{n}\left[v_{i} z_{i} p_{i}^{\prime} 1\left\{q^{\prime} \Sigma z_{i}>0\right\}\right]-\mathrm{E}\left[z_{i} p_{i}^{\prime} 1\left\{q^{\prime} \Sigma z_{i}>0\right\}\right]\right\| \\
& \mu_{2}=\left\|\mathbb{E}_{n}\left[v_{i} z_{i} p_{i}^{\prime}\left\{1\left\{q^{\prime} \Sigma z_{i}>0\right\}-1\left\{q^{\prime} \Sigma z_{i}>0\right\}\right\}\right]\right\| .
\end{aligned}
$$

By Lemma 11, $\|\Sigma-\bar{\Sigma}\|=o_{\mathrm{P}}(1)$, and from Assumption C3 $\left\|\mathrm{E}\left[z_{i} p_{i} 1\left\{q^{\prime} \Sigma z_{i}>0\right\}\right]\right\| \lesssim 1$.

By elementary inequalities

$$
\bar{\mu}_{2}^{2} \leq \mathbb{E}_{n}\left\|v_{i}\right\|^{2} \mathbb{E}_{n}\left\|z_{i}\right\|^{2}\left\|\mathbb{E}_{n}\left[p_{i} p_{i}^{\prime}\right]\right\| \mathbb{E}_{n}\left[\left\{1\left\{q^{\prime} \Sigma z_{i}>0\right\}-1\left\{q^{\prime} \bar{\Sigma} z_{i}>0\right\}\right\}^{2}\right] \lesssim \mathrm{P} n^{-m / 2},
$$

where we used the Chebyshev inequality along with $\mathrm{E}\left\|v_{i}\right\|^{2}=1$ and $\mathrm{E}\left[\left\|z_{i}\right\|^{2}\right]<\infty,\left\|\mathbb{E}_{n}\left[p_{i} p_{i}^{\prime}\right]\right\| \lesssim_{\mathrm{P}}$ 1 by Lemma 11, and $\mathbb{E}_{n}\left[\left\{1\left\{q^{\prime} \Sigma z_{i}>0\right\}-1\left\{q^{\prime} \bar{\Sigma} z_{i}>0\right\}\right\}^{2}\right] \lesssim_{\mathrm{P}} n^{-m / 2}$ by Step 5 below.

We can write $\mu_{1}=\sup _{g \in \mathcal{G}}\left|\mathbb{E}_{n} g-\mathrm{E} g\right|$, where $\mathcal{G}:=\left\{v_{i} \gamma^{\prime} z_{i} p_{i}^{\prime} \eta 1\left\{q^{\prime} \Sigma z_{i}>0\right\},\|\gamma\|=1,\|\eta\|=\right.$ $1\}$. The function class $\mathcal{G}$ obeys

$$
\sup _{Q} \log N\left(\epsilon\|G\|_{Q, 2}, \mathcal{G}, L_{2}(Q)\right) \lesssim\left(\operatorname{dim}\left(z_{i}\right)+\operatorname{dim}\left(p_{i}\right)\right) \log (1 / \epsilon) \lesssim k \log (1 / \epsilon)
$$

for the envelope $G_{i}=v_{i}\|z\|_{i} \cdot \xi_{k}$ that obeys $\max _{i} \log G_{i} \lesssim \mathrm{P} \log n$ by $E\left|v_{i}\right|^{p}<\infty$ for any $p>0, \mathrm{E}\left\|z_{i}\right\|^{2}<\infty$ and $\log \xi_{k} \lesssim_{\mathrm{P}} \log n$. Invoking Lemma 12 we obtain

$$
\mu_{1} \lesssim \mathrm{P} \sqrt{(k / n) \cdot \log n} \times \sup _{g \in \mathcal{G}}\|g\|_{\mathbb{P}_{n}, 2} \vee \sup _{g \in \mathcal{G}}\|g\|_{\mathrm{P}, 2},
$$


where

$$
\begin{gathered}
\sup _{g \in \mathcal{G}}\|g\|_{\mathbb{P}_{n}, 2} \lesssim_{\mathrm{P}}\left(\max _{i}\left\|z_{i}\right\| \max _{i} v_{i} \cdot\left\|\mathbb{E}_{n}\left[p_{i} p_{i}^{\prime}\right]\right\|\right) \wedge\left(\left[\mathbb{E}_{n}\left\|v_{i} z_{i}\right\|^{2}\right]^{1 / 2} \xi_{k}\right) \\
\lesssim_{\mathrm{P}}\left(\max _{i} v_{i} \max _{i}\left\|z_{i}\right\|\right) \wedge \xi_{k} \lesssim_{\mathrm{P}}\left(\log n \max _{i}\left\|z_{i}\right\|\right) \wedge \xi_{k}
\end{gathered}
$$

by $\mathrm{E}\left\|z_{i}\right\|^{2}<\infty$ and by $\mathbb{E}_{n}\left[p_{i} p_{i}^{\prime}\right] \lesssim_{\mathrm{P}} 1, \max _{i} v_{i} \lesssim \mathrm{P} \log n$ and $\sup _{g \in \mathcal{G}}\|g\|_{\mathrm{P}, 2}=\left\|E z_{i} p_{i}^{\prime}\right\| \lesssim 1$ by Assumption C3. Thus

$$
\mu_{1} \lesssim \mathrm{P} \sqrt{(k / n) \cdot \log n}\left(\max _{i}\left\|z_{i}\right\| \log n\right) \wedge \xi_{k},
$$

and the claim of the step follows.

Step 5. Here we show

$$
\sup _{q \in \mathcal{S}^{d-1}} \mathbb{E}_{n}\left[\left(1\left(q^{\prime} \Sigma z_{i}<0\right)-1\left(q^{\prime} \bar{\Sigma} z_{i}<0\right)\right)^{2}\right] \lesssim_{\mathrm{P}} n^{-m / 2}
$$

Note $\left(1\left(q^{\prime} \Sigma z_{i}<0\right)-1\left(q^{\prime} \bar{\Sigma} z_{i}<0\right)\right)^{2}=1\left(q^{\prime} \Sigma z_{i}<0<q^{\prime} \bar{\Sigma} z_{i}\right)+1\left(q^{\prime} \Sigma z_{i}>0>q^{\prime} \bar{\Sigma} z_{i}\right)$. The set

$$
\mathcal{F}=\left\{1\left(q^{\prime} \Sigma z_{i}<0<q^{\prime} \tilde{\Sigma} z_{i}\right)+1\left(q^{\prime} \Sigma z_{i}>0>q^{\prime} \tilde{\Sigma} z_{i}\right), q \in \mathcal{S}^{d-1},\|\Sigma\| \leq M,\|\tilde{\Sigma}\| \leq M\right\}
$$

is P-Donsker because it is a VC class with a constant envelope. Therefore, $\left|\mathbb{E}_{n} f-\mathrm{E} f\right| \lesssim_{\mathrm{P}}$ $n^{-1 / 2}$ uniformly on $f \in \mathcal{F}$. Hence uniformly in $q \in \mathcal{S}^{d-1}, \mathbb{E}_{n}\left[\left(1\left(q^{\prime} \sum z_{i}<0\right)-1\left(q^{\prime} \bar{\Sigma}^{\prime} z_{i}<0\right)\right)^{2}\right]$ is equal to

$$
\begin{aligned}
& \mathrm{E}\left[1\left(q^{\prime} \Sigma z_{i}<0<q^{\prime} \bar{\Sigma}^{\prime} z_{i}\right)+1\left(q^{\prime} \Sigma z_{i}>0>q^{\prime} \bar{\Sigma}^{\prime} z_{i}\right)\right]+O_{\mathrm{P}}\left(n^{-1 / 2}\right) \\
= & \mathrm{P}\left(\left|q^{\prime} \Sigma z_{i}\right|<\left|q^{\prime}(\Sigma-\bar{\Sigma}) z_{i}\right|\right)+O_{\mathrm{P}}\left(n^{-1 / 2}\right) \\
\leq & \|\Sigma-\bar{\Sigma}\|^{m}+O_{\mathrm{P}}\left(n^{-1 / 2}\right) \lesssim_{\mathrm{P}} n^{-m / 2}+n^{-1 / 2} \lesssim_{\mathrm{P}} n^{-m / 2}
\end{aligned}
$$

where we are using that for $0<m \leq 1$

$$
\mathrm{P}\left(\left|q^{\prime} \Sigma z_{i}\right|<\left|q^{\prime}(\Sigma-\bar{\Sigma}) z_{i}\right|\right) \leq \mathrm{P}\left(\left|q^{\prime} \Sigma z_{i} /\left\|z_{i}\right\|\right|<\|q\|\|\Sigma-\bar{\Sigma}\|\right) \lesssim\|\bar{\Sigma}-\Sigma\|^{m}
$$

where the last inequality holds by Assumption C1, which gives that $\mathrm{P}\left(\left|q^{\prime} \Sigma z_{i} /\left\|z_{i}\right\|\right|<\delta\right) / \delta^{m} \lesssim$ 1 .

Lemma 2. Let $w_{i, \mu}(\alpha)=:\left(\theta_{0}(x, \alpha) 1\left(\mu z_{i}<0\right)+\theta_{1}(x, \alpha) 1\left(\mu z_{i} \geq 0\right)\right)$. 1. (Sample) Then uniformly in $t \in T$

$$
\sqrt{n}\left(\widehat{\sigma}_{\theta, \widehat{\Sigma}}-\widehat{\sigma}_{\theta, \Sigma}\right)(t)=\sqrt{n} q^{\prime}(\widehat{\Sigma}-\Sigma) \mathrm{E}\left[z_{i} w_{i, q^{\prime} \Sigma}(\alpha)\right]+o_{\mathrm{P}}(1)
$$

2. (Bootstrap) Then uniformly in $t \in T$

$$
\sqrt{n}\left(\tilde{\sigma}_{\theta, \tilde{\Sigma}}-\tilde{\sigma}_{\theta, \Sigma}\right)(t)=\sqrt{n} q^{\prime}(\tilde{\Sigma}-\Sigma) \mathrm{E}\left[z_{i} w_{i, q^{\prime} \Sigma}(\alpha)\right]+o_{\mathrm{P}}(1)
$$

Proof of Lemma 2. In order to cover both cases with one proof, we will use $\bar{\theta}$ to mean either the unweighted estimator $\widehat{\theta}$ or the weighted estimator $\tilde{\theta}$ and so on, and $v_{i}$ to mean either 1 in the case of the unweighted estimator or exponential weights $e_{i}$ in the case of the 
weighted estimator. We also observe that $\bar{\Sigma} \rightarrow_{\mathrm{P}} \Sigma$ by the law of large numbers (Lemma 11) and the continuous mapping theorem.

Step 1. Define $\mathcal{F}=\left\{q^{\prime} \Sigma z_{i} w_{i, q^{\prime} \Sigma}(t): t \in T,\|\Sigma\| \leq C\right\}$. We have that for $\bar{f}_{i}(t)=q^{\prime} \bar{\Sigma} z_{i} w_{i, q^{\prime}} \bar{\Sigma}(t)$ and $f_{i}(t)=q^{\prime} \Sigma z_{i} w_{i, q^{\prime} \Sigma}(t)$ by definition

$$
\begin{aligned}
\sqrt{n}\left(\bar{\sigma}_{\theta, \bar{\Sigma}}-\bar{\sigma}_{\theta, \Sigma}\right)(t) & =\sqrt{n} \mathbb{E}_{n}\left[\left(v_{i} / \bar{v}\right)\left(\bar{f}_{i}(t)-f_{i}(t)\right)\right] \\
& =\left(\sqrt{n} \mathrm{E}\left[\bar{f}_{i}(t)-f_{i}(t)\right]+\mathbb{G}_{n}\left[v_{i}\left(\bar{f}_{i}(t)-f_{i}(t)\right)^{o}\right]\right) /\left(1+o_{\mathrm{P}}(1)\right) .
\end{aligned}
$$

By intermediate value expansion and Lemma 3, uniformly in $\alpha \in \mathcal{A}$ and $q \in \mathcal{S}^{d-1}$

$\sqrt{n}\left(\mathrm{E}\left[\bar{f}_{i}(t)-f_{i}(t)\right]\right)=\sqrt{n}\left(q^{\prime} \bar{\Sigma}-q^{\prime} \Sigma\right) \mathrm{E}\left[z_{i} w_{i, q^{\prime} \Sigma^{*}(t)}(t)\right]=\sqrt{n}\left(q^{\prime} \bar{\Sigma}-q^{\prime} \Sigma\right) \mathrm{E}\left[z_{i} w_{i, q^{\prime} \Sigma}(t)\right]+o_{\mathrm{P}}(1)$,

for $q \Sigma^{*}(t)$ on the line connecting $q^{\prime} \Sigma$ and $q^{\prime} \bar{\Sigma}$, where the last step follows by the uniform continuity of the mapping $\left(\alpha, q^{\prime} \Sigma\right) \mapsto \mathrm{E}\left[z_{i} w_{i, q^{\prime} \Sigma}(t)\right]$ and $q^{\prime} \bar{\Sigma}-q^{\prime} \Sigma \rightarrow_{\mathrm{P}} 0$. Furthermore $\sup _{t \in T}\left|\mathbb{G}_{n}\left[v_{i}\left(\bar{f}_{i}(t)-f_{i}(t)\right)^{o}\right]\right| \rightarrow_{\mathrm{P}} 0$ by Step 2 below, proving the claim of the Lemma.

Step 2. It suffices to show that for any $t \in T$, we have that $\mathbb{G}_{n}\left[v_{i}\left[\bar{f}_{i}(t)-f_{i}(t)\right]^{o}\right] \rightarrow_{\mathrm{P}} 0$. By Lemma 19.24 from van der Vaart (2000) it follows that if $v_{i}\left[\bar{f}_{i}(t)-f_{i}(t)\right]^{o} \in \mathcal{G}=$ $v_{i}\left((\mathcal{F}-\mathcal{F})^{o}\right)$ is such that

$$
\left(\mathrm{E}\left[\left(v_{i}\left(\bar{f}_{i}(t)-f_{i}(t)\right)^{o}\right)^{2}\right]\right)^{1 / 2} \leq 2\left(\mathrm{E}\left[\left(v_{i}\left(\bar{f}_{i}(t)-f_{i}(t)\right)\right)^{2}\right]\right)^{1 / 2} \rightarrow_{\mathrm{P}} 0,
$$

and $\mathcal{G}$ is P-Donsker, then $\mathbb{G}_{n}\left[v_{i}\left(\bar{f}_{i}(t)-f_{i}(t)\right)^{o}\right] \rightarrow_{\mathrm{P}} 0$. Here $\mathcal{G}$ is P-Donsker because $\mathcal{F}$ is a PDonsker class formed by taking products of $\mathcal{F}_{2} \supseteq\left\{\theta_{i \ell}(\alpha): \alpha \in \mathcal{A}, \ell=0,1\right\}$, which possess a square-integrable envelope, with bounded VC classes $\left\{1\left(q^{\prime} \Sigma z_{i}>0\right), q \in \mathcal{S}^{d-1},\|\Sigma\| \leq\right.$ $C\}$ and $\left\{1\left(q^{\prime} \Sigma z_{i} \leq 0\right), q \in \mathcal{S}^{d-1},\|\Sigma\| \leq C\right\}$ and then summing followed by demeaning. The difference $(\mathcal{F}-\mathcal{F})^{o}$ is also P-Donsker, and its product with the independent squareintegrable variable $v_{i}$ is still a $\mathrm{P}$-Donsker class with a square-integrable envelope. The functions class has a square-integrable envelope. Note that

$$
\begin{aligned}
& \quad \mathrm{E}\left[\bar{f}_{i}(t)-f_{i}(t)\right]^{2}=\mathrm{E}\left(\begin{array}{c} 
\\
\left(q^{\prime} \bar{\Sigma}-q^{\prime} \Sigma\right) z_{i} \theta_{0 i}(\alpha) 1\left(q^{\prime} \bar{\Sigma}^{\prime} z_{i}<0\right) 1\left(q^{\prime} \Sigma z_{i}<0\right) \\
+\left(q^{\prime} \bar{\Sigma}-q^{\prime} \Sigma\right) z_{i} \theta_{1 i}(\alpha) 1\left(q^{\prime} \bar{\Sigma} z_{i}>0\right) 1\left(q^{\prime} \Sigma z_{i}>0\right) \\
+\left(q^{\prime} \bar{\Sigma} z_{i} \theta_{0 i}(\alpha)-q^{\prime} \Sigma z_{i} \theta_{i 1}(\alpha)\right) 1\left(q^{\prime} \bar{\Sigma} z_{i}<0<q^{\prime} \Sigma z_{i}\right) \\
+\left(q^{\prime} \bar{\Sigma} z_{i} \theta_{1 i}(\alpha)-q^{\prime} \Sigma z_{i} \theta_{0 i}(\alpha)\right) 1\left(q^{\prime} \bar{\Sigma} z_{i}>0>q^{\prime} \Sigma z_{i}\right)
\end{array}\right)^{2} \\
& \lesssim\|\| \bar{\Sigma}-\Sigma\left\|^{2}\right\|_{\mathrm{P}, 2} \cdot\|\| z_{i}\left\|^{2}\right\|_{\mathrm{P}, 2} \max _{\alpha \in \mathcal{A}, \ell \in\{0,1\}}\left\|\theta_{\ell i}^{2}(\alpha)\right\| \|_{\mathrm{P}, 2} \\
& +\left(\|\bar{\Sigma}\|_{\mathrm{P}}^{2} \vee\|\Sigma\|^{2}\right) \cdot\|\| z_{i}\left\|^{2}\right\|_{\mathrm{P}, 2} \max _{\alpha \in \mathcal{A}, \ell \in\{0,1\}}\left\|\theta_{\ell i}^{2}(\alpha)\right\|_{\mathrm{P}, 2} \cdot \sup _{q \in \mathcal{S}^{d-1}} \mathrm{P}\left[\left|q^{\prime} \Sigma z_{i}\right|<\left|q^{\prime}(\bar{\Sigma}-\Sigma) z\right|\right]^{1 / 2} \\
& \lesssim \mathrm{P}\|\bar{\Sigma}-\Sigma\|^{2}+\sup _{q \in \mathcal{S}^{d-1}} \mathrm{P}\left[\left|q^{\prime} \Sigma z_{i} /\left\|z_{i}\right\|\right|<\|\bar{\Sigma}-\Sigma\|\right]^{1 / 2} \rightarrow 0,
\end{aligned}
$$

where we invoked the moment and smoothness assumptions.

Lemma 3 (A Uniform Derivative). Let $\sigma_{i, \mu}(\alpha)=\mu z_{i}\left(\theta_{0 i}(\alpha) 1\left(\mu z_{i}<0\right)+\theta_{1 i}(\alpha) 1\left(\mu z_{i}>0\right)\right)$. Uniformly in $\mu \in M=\left\{q^{\prime} \Sigma: q \in \mathcal{S}^{d-1},\|\Sigma\| \leq C\right\}$ and $\alpha \in \mathcal{A}$

$$
\frac{\partial \mathrm{E}\left[\sigma_{i, \mu}(\alpha)\right]}{\partial \mu}=\mathrm{E}\left[z_{i} w_{i, \mu}(\alpha)\right]
$$

where the right hand side is uniformly continuous in $\mu$ and $\alpha$. 
Proof: The continuity of the mapping $(\mu, \alpha) \mapsto \mathrm{E}\left[z_{i} w_{i, \mu}(u)\right]$ follows by an application of the dominated convergence theorem and stated assumptions on the envelopes.

Note that for any $\|\delta\| \rightarrow 0$

$$
\frac{\mathrm{E}\left[(\mu+\delta) z_{i} w_{i, \mu+\delta}(\alpha)\right]-\mathrm{E}\left[\mu z_{i} w_{i, \mu}(\alpha)\right]}{\|\delta\|}=\frac{\delta}{\|\delta\|} \mathrm{E}\left[z_{i} w_{i, \mu}(\alpha)\right]+\frac{1}{\|\delta\|} \mathrm{E}\left[R_{i}(\delta, \mu, \alpha)\right],
$$

where

$$
\begin{aligned}
& R_{i}(\delta, \mu, \alpha):=\quad(\mu+\delta) z_{i}\left(\theta_{1 i}(\alpha)-\theta_{0 i}(\alpha)\right) 1\left(\mu z_{i}<0<(\mu+\delta) z_{i}\right) \\
& +(\mu+\delta) z_{i}\left(\theta_{0 i}(\alpha)-\theta_{1 i}(\alpha)\right) 1\left(\mu z_{i}>0>(\mu+\delta) z_{i}\right) .
\end{aligned}
$$

By Cauchy-Schwarz and the maintained assumptions

$$
\begin{aligned}
\sup _{\mu \in M, \alpha \in \mathcal{A}} \mathrm{E}\left|R_{i}(\delta, \mu, \alpha)\right| & \lesssim\|\delta z\|_{\mathrm{P}, 2} \cdot \sup _{\alpha \in \mathcal{A}, \ell \in\{0,1\}}\left\|\theta_{\ell i}(\alpha)\right\|_{\mathrm{P}, 2} \sup _{\mu \in M, \alpha \in \mathcal{A}}[\mathrm{P}(|\mu z|<|\delta z|)]^{1 / 2} \\
& \lesssim\|\delta z\|_{\mathrm{P}, 2} \cdot 1 \cdot \delta^{m / 2} .
\end{aligned}
$$

Therefore, as $\|\delta\| \rightarrow 0$

$$
\sup _{\mu \in M, \alpha \in \mathcal{A}} \frac{1}{\|\delta\|}\left|\mathrm{E}\left[R_{i}(\delta, \mu, u)\right]\right| \leq \sup _{\mu \in M, \alpha \in \mathcal{A}} \frac{1}{\|\delta\|} \mathrm{E}\left|R_{i}(\delta, \mu, \alpha)\right| \lesssim \delta^{m / 2} \rightarrow 0
$$

Lemma 4 (Coupling Lemma). 1. (Sample) We have that

$$
\mathbb{G}_{n}[h(t)]=\mathbb{G}[h(t)]+o_{\mathrm{P}}(1) \text { in } \ell^{\infty}(T),
$$

where $\mathbb{G}$ is a P-Brownian bridge with covariance function $\mathrm{E}\left[h(t) h\left(t^{\prime}\right)\right]-\mathrm{E}[h(t)] \mathrm{E}\left[h\left(t^{\prime}\right)\right]$.

2. (Bootstrap). We have that

$$
\mathbb{G}_{n}\left[e^{o} h^{o}(t)\right]=\widetilde{\mathbb{G}[h(t)]}+o_{\mathrm{P}}(1) \text { in } \ell^{\infty}(T),
$$

where $\widetilde{\mathbb{G}}$ is a P-Brownian bridge with covariance function $\mathrm{E}\left[h(t) h\left(t^{\prime}\right)\right]-\mathrm{E}[h(t)] \mathrm{E}\left[h\left(t^{\prime}\right)\right]$.

Proof. The proof can be accomplished by using a single common notation. Specifically it will suffice to show that for either the case $g_{i}=1$ or $g_{i}=e_{i}-1$

$$
\mathbb{G}_{n}\left[g h^{o}\right]=\mathbb{G}^{g}[h(t)]+o_{\mathrm{P}}(1) \text { in } \ell^{\infty}(T),
$$

where $\mathbb{G}$ is a P-Brownian bridge with covariance function $\mathrm{E}\left[h(t) h\left(t^{\prime}\right)\right]-\mathrm{E}[h(t)] \mathrm{E}\left[h\left(t^{\prime}\right)\right]$. The process $\mathbb{G}^{g}$ for the case of $g_{i}=1$ is different (in fact independent) of the process $\mathbb{G}^{g}$ for the case of $g_{i}=e_{i}-1$, but they both have identical distributions. Once we understand this, we can drop the index $g$ for the process.

Within this proof, it will be convenient to define:

$$
S_{n}(t):=\mathbb{G}_{n}\left[g h^{o}(t)\right] \text { and } Z_{n}(t):=\mathbb{G}[h(t)] .
$$

Let $B_{j k}, j=1, \ldots, p$ be a partition of $T$ into sets of diameter at most $j^{-1}$. We need at most

$$
p \lesssim j^{d}, \quad d=\operatorname{dim}(T)
$$

such partition sets. Choose $t_{j k}$ as arbitrary points in $B_{j k}$, for all $j=1, \ldots, p$. We define the sequence of projections $\pi_{j}: T \rightarrow T, j=0,1,2, \ldots, \infty$ by $\pi_{j}(t)=t_{j k}$ if $t \in B_{j k}$. 
In what follows, given a process $Z$ in $\ell^{\infty}(T)$ and its projection $Z \circ \pi_{j}$, whose paths are constant over the partition set, we shall identify the process $Z \circ \pi_{j}$ with a random vector $Z \circ \pi_{j}$ in $\mathbb{R}^{p}$, when convenient. Analogously, given a random vector $Z$ in $\mathbb{R}^{p}$ we identify it with a process $Z$ in $\ell^{\infty}(T)$, whose paths are constant over the elements of the partition sets.

The result follows from the following relations proven below:

1. Finite-Dimensional Approximation. As $j / \log n \rightarrow \infty$, then $\Delta_{1}=\sup _{t \in T} \| S_{n}(t)-$ $S_{n} \circ \pi_{j}(t) \| \rightarrow_{\mathrm{P}} 0$.

2. Coupling with a Normal Vector. There exists $\mathcal{N}_{n j}={ }_{d} N\left(0, \operatorname{var}\left[S_{n} \circ \pi_{j}\right]\right)$ such that, if $p^{5} \xi_{k}^{2} / n \rightarrow 0$, then $\Delta_{2}=\sup _{j}\left|\mathcal{N}_{n j}-S_{n} \circ \pi_{j}\right| \rightarrow_{\mathrm{P}} 0$.

3. Embedding a Normal Vector into a Gaussian Process. There exists a Gaussian process $Z_{n}$ with the properties stated in the lemma such that $\mathcal{N}_{n j}=Z_{n} \circ \pi_{j}$ almost surely.

4. Infinite-Dimensional Approximation. if $j \rightarrow \infty$, then $\Delta_{3}=\sup _{t \in T} \mid Z_{n}(t)-Z_{n} \circ$ $\pi_{j}(t) \mid \rightarrow_{\mathrm{P}} 0$.

We can select the sequence $j=\log ^{2} n$ such that the conditions on $j$ stated in relations (1)-(4) hold. We then conclude using the triangle inequality that

$$
\sup _{t \in T}\left|S_{n}(t)-Z_{n}(t)\right| \leq \Delta_{1}+\Delta_{2}+\Delta_{3} \rightarrow_{\mathrm{P}} 0 .
$$

Relation 1 follows from

$$
\Delta_{1}=\sup _{t \in T}\left|S_{n}(t)-S_{n} \circ \pi_{j}(t)\right| \leq \sup _{\left\|t-t^{\prime}\right\| \leq j^{-1}}\left|S_{n}(t)-S_{n}\left(t^{\prime}\right)\right| \rightarrow_{\mathrm{P}} 0,
$$

where the last inequality holds by Lemma 5.

Relation 2 follows from the use of Yurinskii's coupling (Pollard (2002, page 244)): Let $\zeta_{1}, \ldots, \zeta_{n}$ be independent $p$-vectors with $\mathrm{E} \zeta_{i}=0$ for each $i$, and $\kappa:=\sum_{i} \mathrm{E}\left[\left\|\zeta_{i}\right\|^{3}\right]$ finite. Let $S=\zeta_{1}+\cdot+\zeta_{n}$. For each $\delta>0$ there exists a random vector $T$ with a $N(0, \operatorname{var}(S))$ distribution such that

$$
\mathrm{P}\{\|S-T\|>3 \delta\} \leq C_{0} B\left(1+\frac{|\log (1 / B)|}{p}\right) \text { where } B:=\kappa p \delta^{-3},
$$

for some universal constant $C_{0}$.

In order to apply the coupling, we collapse $S_{n} \circ \pi_{j}$ to a $p$-vector, and we let

$$
\zeta_{i}=\zeta_{1 i}+\ldots+\zeta_{4 i} \in \mathbb{R}^{p}, \quad \zeta_{l i}=g_{i} h_{l i}^{o} \circ \pi \in \mathbb{R}^{p},
$$

where $h_{l i}, l=1, \ldots, 4$ are defined in $(\mathrm{B} .1)$, so that $S_{n} \circ \pi_{j}=\sum_{i=1}^{n} \zeta_{i} / \sqrt{n}$. Now note that since $\mathrm{E}\left[\left\|\zeta_{i}\right\|^{3}\right] \lesssim \max _{1 \leq l \leq 4} \mathrm{E}\left[\left\|\zeta_{l i}\right\|^{3}\right]$ and

$$
\begin{aligned}
\mathrm{E}\left\|\zeta_{l i}\right\|^{3} & =p^{3 / 2} \mathrm{E}\left(\frac{1}{p} \sum_{k=1}^{p}\left|g_{i} h_{l i}^{o}\left(t_{k j}\right)\right|^{2}\right)^{3 / 2} \leq p^{3 / 2} \mathrm{E}\left(\frac{1}{p} \sum_{k=1}^{p}\left|g_{i} h_{l i}^{o}\left(t_{k j}\right)\right|^{3}\right) \\
& \leq p^{3 / 2} \sup _{t \in T} \mathrm{E}\left|h_{l i}^{o}\left(t_{k j}\right)\right|^{3} \mathrm{E}\left|g_{i}\right|^{3},
\end{aligned}
$$


where we use the independence of $g_{i}$, we have that

$$
\mathrm{E}\left[\left\|\zeta_{i}\right\|^{3}\right] \lesssim p^{3 / 2} \max _{1 \leq l \leq 4} \sup _{t \in T} \mathrm{E}\left|h_{l i}^{o}(t)\right|^{3} \mathrm{E}\left|g_{i}\right|^{3} .
$$

Next we bound the right side of the display above for each $l$. First, for $A(t):=$ $q^{\prime} \Sigma \mathrm{E}\left[z_{i} p_{i}^{\prime} 1\left\{q^{\prime} \Sigma z_{i}>0\right\}\right] J_{1}^{-1}(\alpha)$

$$
\begin{aligned}
\left.\sup _{t \in T} \mathrm{E} \mid h_{1 i}^{o}(t)\right)\left.\right|^{3} & =\sup _{t \in T} \mathrm{E}\left|A(t) p_{i} \varphi_{i 1}(\alpha)\right|^{3} \leq \sup _{t \in T}\|A(t)\|^{3} \cdot \sup _{\|\delta\|=1} \mathrm{E}\left|\delta^{\prime} p_{i}\right|^{3} \sup _{\alpha \in \mathcal{A}, x \in X} \mathrm{E}\left[\left|\varphi_{i}(\alpha)\right|^{3} \mid x_{i}=x\right] \\
& \lesssim \sup _{\|\delta\|=1} \mathrm{E}\left|\delta^{\prime} p_{i}\right|^{3} \sup _{\alpha \in \mathcal{A}, x \in X} \mathrm{E}\left[\left|\varphi_{i}(\alpha)\right|^{3} \mid x_{i}=x\right] \\
& \lesssim \xi_{k} \sup _{\|\delta\|=1} \mathrm{E}\left|\delta^{\prime} p_{i}\right|^{2} \sup _{\alpha \in \mathcal{A}, x \in X} \mathrm{E}\left[\left|\varphi_{i}(\alpha)\right|^{3} \mid x_{i}=x\right] \lesssim \xi_{k},
\end{aligned}
$$

where we used the assumption that $\sup _{\alpha \in \mathcal{A}, x \in X} \mathrm{E}\left[\left|\varphi_{i}(\alpha)\right|^{3} \mid x_{i}=x\right] \lesssim 1,\left\|\mathrm{E} p_{i} p_{i}\right\| \lesssim 1$, and that

$$
\sup _{t \in T}\|A(t)\| \leq \sup _{\|\delta\|=1}\left[\mathrm{E}\left[z_{i}^{\prime} \delta\right]^{2}\right]^{1 / 2} \sup _{\|\delta\|=1}\left[\mathrm{E}\left[p_{i}^{\prime} \delta\right]^{2}\right]^{1 / 2} \sup _{\alpha \in \mathcal{A}}\left\|J^{-1}(\alpha)\right\| \lesssim 1
$$

where the last bound is true by assumption. Similarly $\left.\mathrm{E} \mid h_{2 i}^{o}(t)\right)\left.\right|^{3} \lesssim \xi_{k}$. Next

$$
\begin{aligned}
\sup _{t \in T} \mathrm{E}\left|h_{3 i}^{o}(t)\right|^{3} & =\sup _{t \in T} \mathrm{E}\left|q^{\prime} \Sigma\left(x_{i} z_{i}^{\prime}\right)^{o} \Sigma \mathrm{E}\left[z_{i}, w_{i, q^{\prime} \Sigma}(\alpha)\right]\right|^{3} \\
& \lesssim \mathrm{E}\left\|\left(x_{i} z_{i}^{\prime}\right)^{o}\right\|^{3} \sup _{t \in T}\left\|\left[\mathrm{E}\left[z_{i} w_{i, q^{\prime} \Sigma}(\alpha)\right]\right]\right\|^{3} \\
& \lesssim\left(\mathrm{E}\left\|\left(x_{i} z_{i}^{\prime}\right)\right\|^{3}+\left\|\mathrm{E}\left(x_{i} z_{i}^{\prime}\right)\right\|^{3}\right)\left(\mathrm{E}\left\|z_{i}\right\|^{2}\right)^{3 / 2} \sup _{\alpha \in \mathcal{A}} \mathrm{E}\left[\left|\theta_{l i}(\alpha)\right|^{2}\right]^{3 / 2} \\
& \lesssim 1,
\end{aligned}
$$

where the last bound follows from assumptions C3. Finally,

$$
\begin{aligned}
\sup _{t \in T}\left[\mathrm{E}\left|h_{4 i}^{o}(t)\right|^{3}\right]^{1 / 3} & =\sup _{t \in T}\left[\mathrm{E}\left|q^{\prime} \Sigma z_{i} w_{i, q^{\prime} \Sigma}(\alpha)\right|^{3}\right]^{1 / 3}+\sup _{t \in T}\left|\mathrm{E} q^{\prime} \Sigma z_{i} w_{i, q^{\prime} \Sigma}(\alpha)\right| \\
& \leq 2 \sup _{t \in T}\left[\mathrm{E}\left|q^{\prime} \Sigma z_{i}\right|^{6}\right]^{1 / 6}\left[\mathrm{E}\left|w_{i, q^{\prime} \Sigma}(\alpha)\right|^{6}\right]^{1 / 6} \\
& \lesssim\left[\mathrm{E}\left|z_{i}\right|^{6}\right]^{1 / 6} \sup _{\alpha \in \mathcal{A}} \mathrm{E}\left[\left|\theta_{l i}(\alpha)\right|^{6}\right]^{1 / 6} \lesssim 1
\end{aligned}
$$

where the last line follows from assumption $\mathrm{C} 3$.

Therefore, by Yurinskii's coupling, observing that in our case by the above arguments $\kappa=\frac{p^{3 / 2} \xi_{k} n}{(\sqrt{n})^{3}}$, for each $\delta>0$ if $p^{5} \xi_{k}^{2} / n \rightarrow 0$,

$$
\mathrm{P}\left\{\left\|\frac{\sum_{i=1}^{n} \zeta_{i}}{\sqrt{n}}-\mathcal{N}_{n, j}\right\| \geq 3 \delta\right\} \lesssim \frac{n p p^{3 / 2} \xi_{k}}{(\delta \sqrt{n})^{3}}=\frac{p^{5 / 2} \xi_{k}}{\left(\delta^{3} n^{1 / 2}\right)} \rightarrow 0 .
$$

This verifies relation (2).

Relation (3) follows from the a.s. embedding of a finite-dimensional random normal vector into a path of a Gaussian process whose paths are continuous with respect to the standard metric $\rho_{2}$, defined in Lemma 6, which is shown e.g., in Belloni and Chernozhukov (2009b). Moreover, since $\rho_{2}$ is continuous with respect to the Euclidian metric on $T$, as 
shown in part 2 of Lemma 6, the paths of the process are continuous with respect to the Euclidian metric as well.

Relation (4) follows from the inequality

$$
\Delta_{3}=\sup _{t \in T}\left|Z_{n}(t)-Z_{n} \circ \pi_{j}(t)\right| \leq \sup _{\left\|t-t^{\prime}\right\| \leq j^{-1}}\left|Z_{n}(t)-Z_{n}\left(t^{\prime}\right)\right| \lesssim_{\mathrm{P}}(1 / j)^{c} \log (1 / j)^{c} \rightarrow 0,
$$

where $0<c \leq 1 / 2$ is defined in Lemma 6. This inequality follows from the entropy inequality for Gaussian processes (Corollary 2.2.8 of van der Vaart and Wellner (1996))

$$
\mathrm{E} \sup _{\rho_{2}\left(t, t^{\prime}\right) \leq \delta}\left|Z_{n}(t)-Z_{n}\left(t_{0}^{\prime}\right)\right| \leq \int_{0}^{\delta} \sqrt{\log N\left(\epsilon, T, \rho_{2}\right)} d \epsilon
$$

and parts 2 and 3 of Lemma 6. From part 2 of Lemma 6 we first conclude that

$$
\log N\left(\epsilon, T, \rho_{2}\right) \lesssim \log (1 / \epsilon),
$$

and second that $\left\|t-t^{\prime}\right\| \leq(1 / j)$ implies $\rho_{2}\left(t, t^{\prime}\right) \leq(1 / j)^{c}$, so that

$$
\mathrm{E} \sup _{\left\|t-t^{\prime}\right\| \leq 1 / j}\left|Z_{n}(t)-Z_{n}\left(t^{\prime}\right)\right| \leq(1 / j)^{c} \log (1 / j)^{c} \text { as } j \rightarrow \infty .
$$

The claimed inequality then follows by Markov inequality.

Lemma 5 (Bounded Oscillations). Let $c$ be as in Lemma 6 .

1. (Sample) For $\epsilon_{n}=o\left((\log n)^{-1 /(2 c)}\right)$, we have that

$$
\sup _{\left\|t-t^{\prime}\right\| \leq \epsilon_{n}}\left|\mathbb{G}_{n}\left[h(t)-h\left(t^{\prime}\right)\right]\right| \rightarrow_{\mathrm{P}} 0 .
$$

2. (Bootstrap). For $\epsilon_{n}=o\left((\log n)^{-1 /(2 c)}\right)$, we have that

$$
\sup _{\left\|t-t^{\prime}\right\| \leq \epsilon_{n}}\left|\mathbb{G}_{n}\left[\left(e_{i}-1\right)\left(h^{o}(t)-h^{o}\left(t^{\prime}\right)\right)\right]\right| \rightarrow_{\mathrm{P}} 0 .
$$

Proof. To show both statements, it will suffice to show that for either the case $g_{i}=1$ or $g_{i}=e_{i}-1$, we have that

$$
\sup _{\left\|t-t^{\prime}\right\| \leq \epsilon_{n}}\left|\mathbb{G}_{n}\left[g_{i}\left(h^{o}(t)-h^{o}\left(t^{\prime}\right)\right)\right]\right| \rightarrow_{\mathrm{P}} 0 .
$$

Step 1. Since

$$
\sup _{\left\|t-t^{\prime}\right\| \leq \epsilon_{n}}\left|\mathbb{G}_{n}\left[g_{i}\left(h^{o}(t)-h^{o}\left(t^{\prime}\right)\right)\right]\right| \lesssim \max _{1 \leq \ell \leq 4} \sup _{\left\|t-t^{\prime}\right\| \leq \epsilon_{n}}\left|\mathbb{G}_{n}\left[g_{i}\left(h_{\ell}^{o}(t)-h_{\ell}^{o}\left(t^{\prime}\right)\right)\right]\right|,
$$

we bound the latter for each $\ell$. Using the results in Lemma 10 that bound the random entropy of $\mathcal{H}_{1}$ and $\mathcal{H}_{2}$ and the results in Lemma 12 we have that for $\ell=1$ and 2

$$
\Delta_{n \ell}=\sup _{\left\|t-t^{\prime}\right\| \leq \epsilon_{n}}\left|\mathbb{G}_{n}\left[g_{i}\left(h_{\ell}^{o}(t)-h_{\ell}^{o}\left(t^{\prime}\right)\right)\right]\right| \lesssim \mathrm{P} \sqrt{\log n} \sup _{\left\|t-t^{\prime}\right\| \leq \epsilon_{n}} \max _{\mathbb{P} \in\left\{\mathrm{P}, \mathbb{P}_{n}\right\}}\left\|g_{i}\left(h_{\ell}^{o}(t)-h_{\ell}^{o}\left(t^{\prime}\right)\right)\right\|_{\mathbb{P}, 2} .
$$

By Lemma 10 that bounds the entropy of $g_{i}\left(\mathcal{H}_{\ell}^{o}-\mathcal{H}_{\ell}^{o}\right)^{2}$ and Lemma 12 we have that for $\ell=1$ or $\ell=2$,

$$
\sup _{\left\|t-t^{\prime}\right\| \leq \epsilon_{n}}\left|\left\|g_{i}\left(h_{\ell}^{o}(t)-h_{\ell}^{o}\left(t^{\prime}\right)\right)\right\|_{\mathbb{P}_{n}, 2}-\left\|g_{i}\left(h_{\ell}^{o}(t)-h_{\ell}^{o}\left(t^{\prime}\right)\right)\right\|_{\mathrm{P}, 2}\right| \lesssim \mathrm{P} \sqrt{\frac{\log n}{n}} \sup _{t \in T} \max _{\mathbb{P} \in\left\{\mathrm{P}, \mathbb{P}_{n}\right\}}\left\|g_{i}^{2} h_{\ell}^{o 2}(t)\right\|_{\mathbb{P}, 2} .
$$


By Step 2 below we have

$$
\sup _{t \in T} \max _{\mathbb{P} \in\left\{\mathrm{P}, \mathbb{P}_{n}\right\}}\left\|g^{2} h_{\ell}^{o 2}(t)\right\|_{\mathbb{P}, 2} \lesssim \mathrm{P} \sqrt{\xi_{k}^{2} \max _{i \leq n} F_{1}^{4} \max _{i}\left|g_{i}\right|^{4}} \lesssim \mathrm{P} \sqrt{\xi_{k}^{2} \max _{i \leq n} F_{1}^{4}(\log n)^{4}}
$$

and by Lemma 6 , $\left\|g\left(h_{\ell}(t)-h_{\ell}\left(t^{\prime}\right)\right)\right\|_{\mathrm{P}, 2} \lesssim\left\|t-t^{\prime}\right\|^{c}$. Putting the terms together we conclude

$$
\Delta_{n \ell} \lesssim_{\mathrm{P}} \sqrt{\log n}\left(\epsilon_{n}^{c}+\sqrt{\frac{\log n}{n}} \xi_{k} \sqrt{\max _{i \leq n} F_{1}^{4}}(\log n)^{2}\right) \rightarrow_{\mathrm{P}} 0,
$$

by assumption and the choice of $\epsilon_{n}$.

For $\ell=3$ and $\ell=4$, by Lemma 10, $g\left(\mathcal{H}_{3}^{o}-\mathcal{H}_{3}^{o}\right)$ and $g\left(\mathcal{H}_{4}^{o}-\mathcal{H}_{4}^{o}\right)$ are P-Donsker, so that

$$
\Delta_{n \ell} \leq \sup _{\rho_{2}\left(t, t_{n}^{\prime}\right) \leq \epsilon_{n}^{c}}\left|\mathbb{G}_{n}\left[g\left(h_{\ell}^{o}(t)-h_{\ell}^{o}\left(t^{\prime}\right)\right)\right]\right| \rightarrow_{\mathrm{P}} 0 .
$$

Step 2. Since $\left\|g^{2} h_{\ell}^{o 2}(t)\right\|_{\mathbb{P}, 2} \leq 2\left\|g^{2} h_{\ell}^{2}(t)\right\|_{\mathbb{P}, 2}+2\left\|g^{2} \mathrm{E}\left[h_{\ell}^{o}(t)\right]^{2}\right\|_{\mathbb{P}, 2}$, for $\mathbb{P} \in\left\{\mathrm{P}, \mathbb{P}_{n}\right\}$, it suffices to bound each term separately.

Uniformly in $t \in T$ for $\ell=1,2$

$$
\begin{aligned}
\mathbb{E}_{n}\left[g h_{\ell}(t)\right]^{4} & \leq \max _{i} g_{i}^{4} \cdot\|\Sigma\|^{4}\left\|\mathbb{E}_{n}\left[z_{i} p_{i} 1\left\{q^{\prime} \Sigma z_{i}<0\right\}\right]\right\|\left\|J_{0}^{-1}(\alpha)\right\| \cdot \sup _{\|\delta\|=1} \mathbb{E}_{n}\left[\left[\delta^{\prime} p_{i}\right]^{4} \varphi_{i \ell}^{4}(\alpha)\right] \\
& \lesssim_{\mathrm{P}}(\log n)^{4} \cdot 1 \cdot \xi_{k}^{2}\left\|\mathbb{E}_{n}\left[p_{i} p_{i}^{\prime}\right]\right\| \max _{i \leq n} F_{1}^{4} \lesssim_{\mathrm{P}} \xi_{k}^{2} \max _{i \leq n} F_{1}^{4}(\log n)^{4}
\end{aligned}
$$

where we used assumptions $\mathrm{C} 3$ and $\mathrm{C} 5$ and the fact that $\left\|\mathbb{E}_{n}\left[z_{i} p_{i} 1\left\{q^{\prime} \Sigma z_{i}<0\right\}\right]\right\| \lesssim_{\mathrm{P}} 1$ and $\mathbb{E}_{n}\left[p_{i} p_{i}^{\prime}\right] \lesssim_{\mathrm{P}} 1$ as shown in the proof of Lemma 1 .

Uniformly in $t \in T$ for $\ell=1,2$

$$
\begin{array}{rl}
\mathrm{E}\left[g h_{\ell}(t)\right]^{4} \leq & \mathrm{E}\left[g^{4}\right]\|\Sigma\|^{4}\left\|\mathrm{E}\left[z_{i} p_{i} 1\left\{q^{\prime} \Sigma z_{i}<0\right\}\right]\right\|\left\|J_{0}^{-1}(\alpha)\right\| \cdot \sup _{\|\delta\|=1} \mathrm{E}\left[\left[\delta^{\prime} p_{i}\right]^{4} \varphi_{i \ell}^{4}(\alpha)\right] \\
\lesssim_{\mathrm{P}} & 1 \cdot \xi_{k}^{2}\left\|\mathrm{E}\left[p_{i} p_{i}^{\prime}\right]\right\| \sup _{x \in X, \alpha \in \mathcal{A}} \mathrm{E}\left[\varphi_{i \ell}^{4}(\alpha) \mid x_{i}=x\right] \lesssim \xi_{k}^{2},
\end{array}
$$

where we used assumption C3.

Uniformly in $t \in T$ for $\ell=1,2$

$$
\mathbb{E}_{n}\left[g^{4} \mathrm{E}\left[h_{\ell}^{o}(t)\right]^{4}\right] \leq \mathbb{E}_{n} g^{4} \mathrm{E}\left[h_{\ell}^{o}(t)\right]^{4} \lesssim_{P} 1 \cdot \mathrm{E}\left[h_{\ell}^{o 2}(t)\right]^{2} \lesssim 1,
$$

and

$$
\mathrm{E}\left[g^{4} \mathrm{E}\left[h_{\ell}^{o}(t)\right]^{4}\right] \leq \mathrm{E} g^{4} \mathrm{E}\left[h_{\ell}^{o}(t)\right]^{4} \lesssim 1 \cdot \mathrm{E}\left[h_{\ell}^{o 2}(t)\right]^{2} \lesssim 1 .
$$

where the bound in $\mathrm{E}\left[h_{\ell}^{o 2}(t)\right]^{2}$ follows from calculations given in the proof of Lemma 6 .

Lemma 6 (Covariance Properties). $\quad$ 1. For some $0<c \leq 1 / 2$

$$
\rho_{2}\left(h(t), h\left(t^{\prime}\right)\right)=\left(\mathrm{E}\left[h(t)-h\left(t^{\prime}\right)\right]^{2}\right)^{1 / 2} \lesssim \rho\left(t, t^{\prime}\right):=\left\|t-t^{\prime}\right\|^{c}
$$

2. The covariance function $\mathrm{E}\left[h(t) h\left(t^{\prime}\right)\right]-\mathrm{E}[h(t)] \mathrm{E}\left[h\left(t^{\prime}\right)\right]$ is equi-continuous on $T \times T$ uniformly in $k$. 
3. A sufficient condition for the variance function to be bounded away from zero, $\inf _{t \in T} \operatorname{var}(h(t)) \geq L>0$, uniformly in $k$ is that the following matrices have minimal eigenvalues bounded away from zero uniformly in $k: \operatorname{var}\left(\left[\begin{array}{lll}\varphi_{i 1}(\alpha) & \varphi_{i 0}(\alpha)\end{array}\right]^{\prime} \mid x_{i}, z_{i}\right)$ $\mathrm{E}\left[p_{i} p_{i}^{\prime}\right], J_{0}^{-1}(\alpha), J_{1}^{-1}(\alpha), b_{0}^{\prime} b_{0}$, and $b_{1}^{\prime} b_{1}$, where $b_{1}=\mathrm{E}\left[z_{i} p_{i}^{\prime} 1\left\{q^{\prime} \Sigma z_{i}>0\right\}\right]$ and $b_{0}=\mathrm{E}\left[z_{i} p_{i}^{\prime} 1\left\{q^{\prime} \Sigma z_{i}<0\right\}\right]$.

Comment B.2. We emphasize that claim 3 only gives sufficient conditions for $\operatorname{var}(h(t))$ to be bounded away from zero. In particular, the assumption that

$$
\operatorname{mineig}\left(\operatorname{var}\left(\left[\varphi_{i 1}(\alpha) \varphi_{i 0}(\alpha)\right]^{\prime} \mid x_{i}, z_{i}\right)\right) \geq L
$$

is not necessary, and does not hold in all relevant situations. For example, when the upper and lower bounds have first-order equivalent asymptotics, which can occur in the pointidentified and local to point-identified cases, this condition fails. However, the result still follows from equation (B.4) under the assumption that

$$
\operatorname{var}\left(\varphi_{i 1}(\alpha) \mid x_{i}, z_{i}\right)=\operatorname{var}\left(\varphi_{i 0}(\alpha) \mid x_{i}, z_{i}\right) \geq L
$$

Proof. Claim 1. Observe that $\rho_{2}(h(t), h(\tilde{t})) \lesssim \max _{j} \rho_{2}\left(h_{j}(t), h_{j}(\tilde{t})\right)$. We will bound each of these four terms. For the first term, we have

$$
\begin{aligned}
\rho_{2}\left(h_{1}(t), h_{1}(\tilde{t})\right)= & \mathrm{E}\left[\left(\begin{array}{c}
q^{\prime} \Sigma \mathrm{E}\left[z_{i} p_{i}^{\prime} 1\left\{q^{\prime} \Sigma z_{i}>0\right\}\right] J_{1}^{-1}(\alpha) p_{i} \varphi_{i 1}(\alpha)- \\
-\tilde{q}^{\prime} \Sigma \mathrm{E}\left[z_{i} p_{i}^{\prime} 1\left\{\tilde{q}^{\prime} \Sigma z_{i}>0\right\}\right] J_{1}^{-1}(\tilde{\alpha}) p_{i} \varphi_{i 1}(\tilde{\alpha})
\end{array}\right)^{2}\right]^{1 / 2} \\
\leq & \mathrm{E}\left[\left((q-\tilde{q})^{\prime} \Sigma \mathrm{E}\left[z_{i} p_{i}^{\prime} 1\left\{q^{\prime} \Sigma z_{i}>0\right\}\right] J_{1}^{-1}(\alpha) p_{i} \varphi_{i 1}(\alpha)\right)^{2}\right]^{1 / 2}+ \\
& +\mathrm{E}\left[\left(\tilde{q}^{\prime} \Sigma\left(\begin{array}{c}
\mathrm{E}\left[z_{i} p_{i}^{\prime} 1\left\{q^{\prime} \Sigma z_{i}>0\right\}\right]- \\
-\mathrm{E}\left[z_{i} p_{i}^{\prime} 1\left\{\tilde{q}^{\prime} \Sigma z_{i}>0\right\}\right]
\end{array}\right) J_{1}^{-1}(\alpha) p_{i} \varphi_{i 1}(\alpha)\right)^{2}\right]^{1 / 2}+ \\
& +\mathrm{E}\left[\left(\tilde{q}^{\prime} \Sigma \mathrm{E}\left[z_{i} p_{i}^{\prime} 1\left\{\tilde{q}^{\prime} \Sigma z_{i}>0\right\}\right]\left(J_{1}^{-1}(\alpha)-J_{1}^{-1}(\tilde{\alpha})\right) p_{i} \varphi_{i 1}(\alpha)\right)^{2}\right]^{1 / 2}+ \\
& +\mathrm{E}\left[\left(\tilde{q}^{\prime} \Sigma \mathrm{E}\left[z_{i} p_{i}^{\prime} 1\left\{\tilde{q}^{\prime} \Sigma z_{i}>0\right\}\right] J_{1}^{-1}(\tilde{\alpha}) p_{i}\left(\varphi_{i 1}(\alpha)-\varphi_{i 1}(\tilde{\alpha})\right)\right)^{2}\right]^{1 / 2}
\end{aligned}
$$

For the first term we have

$$
\begin{aligned}
& \mathrm{E}\left[\left((q-\tilde{q})^{\prime} \Sigma \mathrm{E}\left[z_{i} p_{i}^{\prime} 1\left\{q^{\prime} \Sigma z_{i}>0\right\}\right] J_{1}^{-1}(\alpha) p_{i} \varphi_{i 1}(\alpha)\right)^{2}\right]^{1 / 2} \leq \\
& \quad \leq\|q-\tilde{q}\|\|\Sigma\|\left\|\mathrm{E}\left[z_{i} p_{i}^{\prime}\right]\right\|\left\|J_{1}^{-1}(\alpha)\right\| \mathrm{E}\left[\left\|p_{i} p_{i}^{\prime}\right\|^{2}\right]^{1 / 2} \sup _{x_{i}, z_{i}} \mathrm{E}\left[\varphi_{i \ell}(\alpha)^{4} \mid x_{i}, z_{i}\right]^{1 / 2}
\end{aligned}
$$

By assumption $\mathrm{C} 3,\left\|\mathrm{E}\left[z_{i} p_{i}^{\prime}\right]\right\|,\left\|J_{1}^{-1}(\alpha)\right\|, \mathrm{E}\left[\left\|p_{i} p_{i}^{\prime}\right\|^{2}\right]$, and $\sup _{x_{i}, z_{i}} \mathrm{E}\left[\varphi_{i 1}(\alpha)^{4} \mid x_{i}, z_{i}\right]$ are bounded uniformly in $k$ and $\alpha$. Therefore,

$$
\mathrm{E}\left[\left((q-\tilde{q})^{\prime} \Sigma \mathrm{E}\left[z_{i} p_{i}^{\prime} 1\left\{q^{\prime} \Sigma z_{i}>0\right\}\right] J_{1}^{-1}(\alpha) p_{i} \varphi_{i 1}(\alpha)\right)^{2}\right]^{1 / 2} \lesssim\|q-\tilde{q}\|
$$


The same conditions give the following bound on the second term.

$$
\begin{aligned}
& \mathrm{E}\left[\left(\tilde{q}^{\prime} \Sigma\left(\begin{array}{c}
\mathrm{E}\left[z_{i} p_{i}^{\prime} 1\left\{q^{\prime} \Sigma z_{i}>0\right\}\right]- \\
-\mathrm{E}\left[z_{i} p_{i}^{\prime} 1\left\{\tilde{q}^{\prime} \Sigma z_{i}>0\right\}\right]
\end{array}\right) J_{1}^{-1}(\alpha) p_{i} \varphi_{i 1}(\alpha)\right)^{2}\right]^{1 / 2} \\
& \quad \lesssim\left\|\mathrm{E}\left[1\left\{q^{\prime} \Sigma z_{i}>0\right\}-1\left\{\tilde{q}^{\prime} \Sigma z_{i}>0\right\}\right]\right\| \\
& \quad \lesssim \mathrm{E}\left[\left(1\left\{q^{\prime} \Sigma z_{i}>0\right\}-1\left\{\tilde{q}^{\prime} \Sigma z_{i}>0\right\}\right)^{2}\right]^{1 / 2}
\end{aligned}
$$

As in step 5 of the proof of Lemma 1, the assumption that $\mathrm{P}\left(\left|q^{\prime} \Sigma z_{i} /\left\|z_{i}\right\|\right|<\delta\right) / \delta^{m} \lesssim 1$ implies

$$
\mathrm{E}\left[\left(1\left\{q^{\prime} \Sigma z_{i}>0\right\}-1\left\{\tilde{q}^{\prime} \Sigma z_{i}>0\right\}\right)^{2}\right]^{1 / 2} \lesssim\|q-\tilde{q}\|^{m / 2}
$$

Similarly, the third term is bounded as follows:

$$
\mathrm{E}\left[\left(\tilde{q}^{\prime} \Sigma \mathrm{E}\left[z_{i} p_{i}^{\prime} 1\left\{\tilde{q}^{\prime} \Sigma z_{i}>0\right\}\right]\left(J_{1}^{-1}(\alpha)-J_{1}^{-1}(\tilde{\alpha})\right) p_{i} \varphi_{i 1}(\alpha)\right)^{2}\right]^{1 / 2} \lesssim\left\|J_{1}^{-1}(\alpha)-J_{1}^{-1}(\tilde{\alpha})\right\|
$$

Note that $J_{1}^{-1}(\alpha)$ is uniformly Lipschitz in $\alpha \in \mathcal{A}$ by assumption $\mathrm{C} 3$, so $\left\|J_{1}^{-1}(\alpha)-J_{1}^{-1}(\tilde{\alpha})\right\| \lesssim$ $\|\alpha-\tilde{\alpha}\|$. Finally, the fourth term is bounded by

$$
\begin{aligned}
& \mathrm{E}\left[\left(\tilde{q}^{\prime} \Sigma \mathrm{E}\left[z_{i} p_{i}^{\prime} 1\left\{\tilde{q}^{\prime} \Sigma z_{i}>0\right\}\right] J_{1}^{-1}(\tilde{\alpha}) p_{i}\left(\varphi_{i 1}(\alpha)-\varphi_{i 1}(\tilde{\alpha})\right)\right)^{2}\right]^{1 / 2} \lesssim \\
& \quad \lesssim \sup _{x_{i}, z_{i}} \mathrm{E}\left[\left(\varphi_{i 1}(\alpha)-\varphi_{i 1}(\tilde{\alpha})\right)^{4} \mid x_{i}, z_{i}\right]^{1 / 2} \\
& \quad \lesssim\|\alpha-\tilde{\alpha}\|^{\gamma_{\varphi}}
\end{aligned}
$$

where we used the assumption that $\mathrm{E}\left[\left(\varphi_{i 1}(\alpha)-\varphi_{i 1}(\tilde{\alpha})\right)^{4} \mid x_{i}, z_{i}\right]^{1 / 2}$ is uniformly $\gamma_{\varphi}$-Hölder continuous in $\alpha$. Combining, we have

$$
\begin{aligned}
\rho_{2}\left(h_{1}(t), h_{1}(\tilde{t})\right) & \lesssim\|q-\tilde{q}\|+\|q-\tilde{q}\|^{m / 2}+\|\alpha-\tilde{\alpha}\|+\|\alpha-\tilde{\alpha}\|^{\gamma_{\varphi}} \\
& \lesssim\left\|t-t^{\prime}\right\|^{1 \wedge m / 2 \wedge \gamma_{\varphi}}
\end{aligned}
$$

An identical argument shows that $\rho_{2}\left(h_{2}(t), h_{2}\left(t^{\prime}\right)\right) \lesssim\left\|t-t^{\prime}\right\|^{1 \wedge m / 2 \wedge \gamma_{\varphi}}$. 
The third and fourth components of $h(t)$ can be bounded using similar arguments. For $h_{3}(t)$, we have

$$
\begin{aligned}
\rho_{2}\left(h_{3}(t), h_{3}(\tilde{t})\right)= & \mathrm{E}\left[\left(q^{\prime} \Sigma x_{i} z_{i}^{\prime} \Sigma \mathrm{E}\left[z_{i} w_{i, q^{\prime} \Sigma}(\alpha)\right]-\tilde{q}^{\prime} \Sigma x_{i} z_{i}^{\prime} \Sigma \mathrm{E}\left[z_{i} w_{i, \tilde{q}^{\prime} \Sigma}(\tilde{\alpha})\right]\right)^{2}\right]^{1 / 2} \\
\lesssim & \mathrm{E}\left[\left((q-\tilde{q})^{\prime} \Sigma x_{i} z_{i}^{\prime} \Sigma \mathrm{E}\left[z_{i} w_{i, q^{\prime} \Sigma}(\alpha)\right]\right)^{2}\right]^{1 / 2}+ \\
& +\mathrm{E}\left[\left(\tilde{q}^{\prime} \Sigma x_{i} z_{i}^{\prime} \Sigma\left(\mathrm{E}\left[z_{i} w_{i, q^{\prime} \Sigma}(\alpha)\right]-\mathrm{E}\left[z_{i} w_{i, \tilde{q}^{\prime} \Sigma}(\alpha)\right]\right)\right)^{2}\right]^{1 / 2}+ \\
& +\mathrm{E}\left[\left(\tilde{q}^{\prime} \Sigma x_{i} z_{i}^{\prime} \Sigma\left(\mathrm{E}\left[z_{i} w_{i, \tilde{q}^{\prime} \Sigma}(\alpha)\right]-\mathrm{E}\left[z_{i} w_{i, \tilde{q}^{\prime} \Sigma}(\tilde{\alpha})\right]\right)\right)^{2}\right]^{1 / 2} \\
\lesssim & \|q-\tilde{q}\|\|\Sigma\| \mathrm{E}\left[z_{i}^{\prime} z_{i} \max _{\ell \in\{0,1\}} \theta_{\ell}\left(x_{i}, \alpha\right)^{2}\right]^{1 / 2}+ \\
& +\|\Sigma\| \mathrm{E}\left[z_{i}^{\prime} z_{i}\left(\theta_{1}\left(x_{i}, \alpha\right)-\theta_{0}\left(x_{i}, \alpha\right)\right)^{2} 1\left\{\left|(q-\tilde{q})^{\prime} \Sigma z_{i}\right| \geq\left|q^{\prime} \Sigma z_{i}\right|\right\}\right]^{1 / 2}+ \\
& +\|\Sigma\| \mathrm{E}\left[z_{i}^{\prime} z_{i} \max _{\ell \in\{0,1\}}\left(\theta_{\ell}\left(x_{i}, \alpha\right)-\theta_{\ell}\left(x_{i}, \tilde{\alpha}\right)\right)^{2}\right]^{1 / 2}
\end{aligned}
$$

By assumption, $\mathrm{E}\left[z_{i}^{\prime} z_{i} \theta_{\ell}\left(x_{i}, \alpha\right)^{2}\right] \leq\left(\mathrm{E}\left[\left\|z_{i}\right\|^{4}\right] \mathrm{E}\left[\theta_{\ell}\left(x_{i}, \alpha\right)^{4}\right]\right)^{1 / 2}$ is bounded uniformly in $\alpha$. Also,

$$
\begin{aligned}
& \mathrm{E}\left[z_{i}^{\prime} z_{i}\left(\theta_{1}\left(x_{i}, \alpha\right)-\theta_{0}\left(x_{i}, \alpha\right)\right)^{2} 1\left\{\left|q^{\prime} \Sigma z_{i}\right| /\left\|z_{i}\right\| \leq\|q-\tilde{q}\|\right\}\right] \lesssim \\
& \quad \lesssim \mathrm{E}\left[\left\|z_{i}\right\|^{4}\right]^{1 / 2}\left(\mathrm{E}\left[\theta_{1}\left(x_{i}, \alpha\right)^{4}\right]^{1 / 2}+\mathrm{E}\left[\theta_{0}\left(x_{i}, \alpha\right)^{4}\right]^{1 / 2}\right) \mathrm{E}\left[1\left\{\left|q^{\prime} \Sigma z_{i}\right| /\left\|z_{i}\right\| \leq\|q-\tilde{q}\|\right\}\right]^{1 / 2} \\
& \quad \lesssim\|q-\tilde{q}\|^{m / 2}
\end{aligned}
$$

where we have used the smoothness condition (C1) and the fact that $\mathrm{E}\left[\left\|z_{i}\right\|^{4}\right]<\infty$ and $\mathrm{E}\left[\theta_{\ell}\left(x_{i}, \alpha\right)^{4}\right]<\infty$ uniformly in $\alpha$.

By assumption, $\theta_{\ell}(x, \alpha)$ are Hölder continuous in $\alpha$ with coefficient $L(x)$, so

$$
\begin{aligned}
\mathrm{E}\left[z_{i}^{\prime} z_{i} \max _{\ell \in\{0,1\}}\left(\theta_{\ell}\left(x_{i}, \alpha\right)-\theta_{\ell}\left(x_{i}, \tilde{\alpha}\right)\right)^{2}\right]^{1 / 2} & \lesssim \mathrm{E}\left[\left\|z_{i}\right\|^{4}\right]^{1 / 2} \mathrm{E}\left[L\left(x_{i}\right)^{4}\right]^{1 / 2}\|\alpha-\tilde{\alpha}\|^{\gamma_{\theta}} \\
& \lesssim\|\alpha-\tilde{\alpha}\|^{\gamma_{\theta}}
\end{aligned}
$$

Thus,

$$
\begin{aligned}
\rho_{2}\left(h_{3}(t), h_{3}(\tilde{t})\right) & \lesssim\|q-\tilde{q}\|+\|q-\tilde{q}\|^{m / 2}+\|\alpha-\tilde{\alpha}\|^{\gamma_{\theta}} \\
& \lesssim\left\|t-t^{\prime}\right\|^{1 \wedge m / 2 \wedge \gamma_{\theta}}
\end{aligned}
$$


For $h_{4}$, we have

$$
\begin{aligned}
\rho_{2}\left(h_{4}(t), h_{4}(\tilde{t})\right)= & \mathrm{E}\left[\left(q^{\prime} \Sigma z_{i} w_{i, q^{\prime} \Sigma}(\alpha)-\tilde{q}^{\prime} \Sigma z_{i} w_{i, \tilde{q}^{\prime} \Sigma}(\tilde{\alpha})\right)^{2}\right]^{1 / 2} \\
\leq & \mathrm{E}\left[\left((q-\tilde{q})^{\prime} \Sigma z_{i} w_{i, q^{\prime} \Sigma}(\alpha)\right)^{2}\right]^{1 / 2}+ \\
& +\mathrm{E}\left[\left(\tilde{q}^{\prime} \Sigma z_{i}\left(w_{i, q^{\prime} \Sigma}(\alpha)-w_{i, \tilde{q}^{\prime} \Sigma}(\alpha)\right)\right)^{2}\right]^{1 / 2}+ \\
& +\mathrm{E}\left[\left(\tilde{q}^{\prime} \Sigma z_{i}\left(w_{i, \tilde{q}^{\prime} \Sigma}(\alpha)-w_{i, \tilde{q}^{\prime} \Sigma}(\tilde{\alpha})\right)\right)^{2}\right]^{1 / 2} \\
\lesssim & \|q-\tilde{q}\|+\|q-\tilde{q}\|^{m / 2}+\|\alpha-\tilde{\alpha}\|^{\gamma_{\theta}}
\end{aligned}
$$

by the exact same arguments used for $h_{3}$.

Claim 2. It suffices to show that $\mathrm{E}\left[h_{j}(t)\right]$ for $j=1, \ldots, 4$ and $\mathrm{E}\left[h_{j}(t) h_{k}\left(t^{\prime}\right)\right]$ for $j=1, \ldots, 4$ and $k=1, \ldots, 4$ are uniformly equicontinuous. Hölder continuity implies equicontinuity, so we show that each of these functions are uniformly Hölder continuous.

Jensen's inequality and the result in Part 1 show that $\mathrm{E}\left[h_{j}(t)\right]$ are uniformly Hölder.

$$
\left|\mathrm{E}\left[h_{j}(t)\right]-\mathrm{E}\left[h_{j}\left(t^{\prime}\right)\right]\right| \leq \mathrm{E}\left[\left(h_{j}(t)-h_{j}\left(t^{\prime}\right)\right)^{2}\right]^{1 / 2} \lesssim\left\|t-t^{\prime}\right\|^{c}
$$

Given this, a simple calculation shows that $\mathrm{E}\left[h_{j}\left(t_{1}\right) h_{k}\left(t_{2}\right)\right]$ are uniformly Hölder as well.

$$
\begin{aligned}
\left|\mathrm{E}\left[h_{j}\left(t_{1}\right) h_{k}\left(t_{2}\right)-h_{j}\left(t_{1}^{\prime}\right) h_{k}\left(t_{2}^{\prime}\right)\right]\right|= & \left|\mathrm{E}\left[\begin{array}{c}
\left(h_{j}\left(t_{1}\right)-h_{j}\left(t_{1}^{\prime}\right)\right) h_{k}\left(t_{2}\right)+ \\
+h_{j}\left(t_{1}^{\prime}\right)\left(h_{k}\left(t_{2}\right)-h_{k}\left(t_{2}^{\prime}\right)\right)
\end{array}\right]\right| \\
\leq & \mathrm{E}\left[\left(h_{j}\left(t_{1}\right)-h_{j}\left(t_{1}^{\prime}\right)\right)^{2}\right]^{1 / 2} \mathrm{E}\left[h_{k}\left(t_{2}\right)^{2}\right]^{1 / 2}+ \\
& +\mathrm{E}\left[h_{j}\left(t_{1}^{\prime}\right)^{2}\right]^{1 / 2} \mathrm{E}\left[\left(h_{k}\left(t_{2}\right)-h_{k}\left(t_{2}^{\prime}\right)\right)^{2}\right]^{1 / 2} \\
\lesssim & \left\|t_{1}-t_{1}^{\prime}\right\|^{c} \vee\left\|t_{2}-t_{2}^{\prime}\right\|^{c}
\end{aligned}
$$

Claim 3. By the law of total variance,

$$
\operatorname{var}(h(t))=\mathrm{E}\left[\operatorname{var}\left(h(t) \mid x_{i}, z_{i}\right)\right]+\operatorname{var}\left(\mathrm{E}\left[h(t) \mid x_{i}, z_{i}\right]\right) .
$$

Note that $h_{3}(t)$ and $h_{4}(t)$ are constant conditional on $x_{i}, z_{i}$, so

$$
\begin{aligned}
\operatorname{var}\left(h(t) \mid x_{i}, z_{i}\right)= & \operatorname{var}\left(h_{1}(t)+h_{2}(t) \mid x_{i}, z_{i}\right) \\
= & {\left[\begin{array}{l}
q^{\prime} \Sigma \mathrm{E}\left[z_{i} p_{i}^{\prime} 1\left\{q^{\prime} \Sigma z_{i}>0\right\}\right] J_{1}^{-1}(\alpha) p_{i} \\
q^{\prime} \Sigma \mathrm{E}\left[z_{i} p_{i}^{\prime} 1\left\{q^{\prime} \Sigma z_{i}<0\right\}\right] J_{0}^{-1}(\alpha) p_{i}
\end{array}\right] \operatorname{var}\left(\left[\begin{array}{l}
\varphi_{i 1}(\alpha) \\
\varphi_{i 0}(\alpha)
\end{array}\right] \mid x_{i}, z_{i}\right) \times } \\
& \times\left[\begin{array}{l}
q^{\prime} \Sigma \mathrm{E}\left[z_{i} p_{i}^{\prime} 1\left\{q^{\prime} \Sigma z_{i}>0\right\}\right] J_{1}^{-1}(\alpha) p_{i} \\
q^{\prime} \Sigma \mathrm{E}\left[z_{i} p_{i}^{\prime} 1\left\{q^{\prime} \Sigma z_{i}<0\right\}\right] J_{0}^{-1}(\alpha) p_{i}
\end{array}\right]
\end{aligned}
$$

Recall that $b_{1}=\mathrm{E}\left[z_{i} p_{i}^{\prime} 1\left\{q^{\prime} \Sigma z_{i}>0\right\}\right]$ and $b_{0}=\mathrm{E}\left[z_{i} p_{i}^{\prime} 1\left\{q^{\prime} \Sigma z_{i}<0\right\}\right]$. Let $\gamma_{\ell}=q^{\prime} \Sigma b_{\ell}$, and mineig $(M)$ denote the minimal eigenvalue of any matrix $M$. By assumption,

$$
\operatorname{mineig}\left(\operatorname{var}\left(\left[\varphi_{i 1}(\alpha) \varphi_{i 0}(\alpha)\right]^{\prime} \mid x_{i}, z_{i}\right)\right)>L,
$$


SO

$$
\begin{aligned}
\mathrm{E}\left[\operatorname{var}\left(h(t) \mid x_{i}, z_{i}\right)\right] & \gtrsim \mathrm{E}\left[\left\|\left[\gamma_{1} J_{1}^{-1}(\alpha) p_{i} \quad \gamma_{0} J_{0}^{-1}(\alpha) p_{i}\right]\right\|^{2}\right] \\
& \gtrsim \mathrm{E}\left[\left\|\gamma_{1} J_{1}^{-1}(\alpha) p_{i}\right\|^{2} \vee\left\|\gamma_{0} J_{0}^{-1}(\alpha) p_{i}\right\|^{2}\right] \\
& \gtrsim \mathrm{E}\left[\left\|\gamma_{1} J_{1}^{-1}(\alpha) p_{i}\right\|^{2}\right] \vee \mathrm{E}\left[\left\|\gamma_{0} J_{0}^{-1}(\alpha) p_{i}\right\|^{2}\right]
\end{aligned}
$$

Repeated use of the inequality $\|x y\|^{2} \geq \operatorname{mineig}\left(y y^{\prime}\right)\|x\|^{2}$ yields for $l=0,1$,

$$
\begin{aligned}
\mathrm{E}\left[\left\|\gamma_{\ell} J_{\ell}^{-1}(\alpha) p_{i}\right\|^{2}\right] & \geq \operatorname{mineig}\left(\mathrm{E}\left[p_{i} p_{i}^{\prime}\right]\right) \operatorname{mineig}\left(J_{\ell}^{-1}(\alpha)\right)^{2}\left\|\gamma_{\ell}\right\|^{2} \\
& \gtrsim \operatorname{mineig}\left(b_{\ell}^{\prime} b_{\ell}\right)\left\|q^{\prime} \Sigma\right\|^{2} \\
& \gtrsim b_{\ell}^{\prime} b_{\ell}
\end{aligned}
$$

where the last line follows from the fact that $b_{\ell}^{\prime} b_{\ell}$ is a scalar. We now show that $b_{\ell}^{\prime} b_{\ell}>0$. Let $f_{1 i}=z_{i} 1\left\{q^{\prime} \Sigma z_{i}>0\right\}$ and $f_{0 i}=z_{i} 1\left\{q^{\prime} \Sigma z_{i}<0\right\}$. Observe that $z_{i}=f_{1 i}+f_{0 i}$ and $\mathrm{E}\left[f_{1 i}^{\prime} f_{0 i}\right]=0$, so

$$
\mathrm{E}\left[f_{1 i}^{\prime} f_{1 i}\right] \vee \mathrm{E}\left[f_{0 i}^{\prime} f_{0 i}\right] \geq \frac{1}{2} \mathrm{E}\left[z_{i}^{\prime} z_{i}\right]>0
$$

By the completeness of our series functions, we can represent $f_{1 i}$ and $f_{0 i}$ in terms of the series functions. Let

$$
f_{1 i}=\sum_{j=1}^{\infty} c_{1 j} p_{j i} f_{0 i}=\sum_{j=1}^{\infty} c_{0 j} p_{j i}
$$

Without loss of generality, assume the series functions are orthonormal. Then

$$
\mathrm{E}\left[f_{1 i}^{\prime} f_{1 i}\right]=\sum_{j=1}^{\infty} c_{1 j}^{2} \mathrm{E}\left[f_{0 i}^{\prime} f_{0 i}\right]=\sum_{j=1}^{\infty} c_{0 j}^{2}
$$

Also,

$$
b_{\ell}^{\prime} b_{\ell}=\sum_{j=1}^{k} c_{\ell j}^{2}
$$

Thus,

$$
\mathrm{E}\left[\operatorname{var}\left(h(t) \mid x_{i}, z_{i}\right)\right] \gtrsim \operatorname{mineig}\left(b_{1}^{\prime} b_{1}\right) \vee \operatorname{mineig}\left(b_{0}^{\prime} b_{0}\right)>0
$$

Lemma 7. Suppose Conditions C1 C5 hold, with $x$ replaced by $(x, v)$ and the supremum over $x \in X$ replaced by the supremum over $(x, v) \in X \times \mathcal{V}$. Let $\mathcal{V}$ be a finite set. Let $\widehat{\vartheta}_{k \ell}=\arg \min _{\vartheta} \mathbb{E}_{n}\left[m\left(y_{i \ell}, p_{k}\left(x_{i}, v_{i}\right)^{\prime} \vartheta, \alpha\right)\right]$. To simplify notation, let $\ell=1$ so that we focus on the upper bounding function. Let

$$
v_{1}^{*}=\arg \min _{v \in \mathcal{V}} \theta_{1}(x, v, \alpha)
$$

and assume that $v_{1}^{*}$ is the unique minimizer of $\theta_{1}(x, v, \alpha)$. Let

$$
\widehat{v}_{1 n} \in \arg \min _{v \in \mathcal{V}} p_{k}\left(x_{i}, v_{i}\right)^{\prime} \widehat{\vartheta}_{k 1}
$$


Let $\vartheta_{k \ell}=\arg \min _{\vartheta} \mathrm{E}\left[m\left(y_{i \ell}, p_{k}\left(x_{i}, v_{i}\right)^{\prime} \vartheta, \alpha\right)\right]$ and assume that

$$
\sup _{(x, v) \in X \times \mathcal{V}, \alpha \in \mathcal{A}}\left|p_{k}(x, v, \alpha) \vartheta_{k 1}-\theta_{1}(x, v, \alpha)\right|=O\left(k^{-r}\right)
$$

for some $r>0.14$ Then the estimator $p_{k}\left(x_{i}, \widehat{v}_{1 n}\right)^{\prime} \widehat{\vartheta}_{k 1}$ satisfies Condition C2.

Proof. We show that $\sqrt{n}\left(p_{k}\left(x_{i}, \widehat{v}_{1 n}\right)^{\prime} \widehat{\vartheta}_{1}-\theta_{1}\left(x, v_{1}^{*}, \alpha\right)\right)$ satisfies equation 4.1). To simplify notation, omit dependence of $\widehat{\vartheta}, \vartheta$ on $k$ in what follows. Observe that:

$$
\begin{aligned}
& \sqrt{n}\left(p_{k}\left(x_{i}, \widehat{v}_{1 n}\right)^{\prime} \widehat{\vartheta}_{1}-\theta_{1}\left(x, v_{1}^{*}, \alpha\right)\right) \\
= & \sqrt{n}\left(p_{k}\left(x_{i}, \widehat{v}_{1 n}\right)^{\prime} \widehat{\vartheta}_{1}-p_{k}\left(x_{i}, v_{1}^{*}\right)^{\prime} \vartheta_{1}\right)+\sqrt{n}\left(p_{k}\left(x_{i}, v_{1}^{*}\right)^{\prime} \vartheta_{1}-\theta_{1}\left(x, v_{1}^{*}, \alpha\right)\right) \\
= & \sqrt{n}\left(p_{k}\left(x_{i}, \widehat{v}_{1 n}\right)^{\prime} \widehat{\vartheta}_{1}-p_{k}\left(x_{i}, v_{1}^{*}\right)^{\prime} \vartheta_{1}\right)+O\left(n^{1 / 2} k^{-r}\right) \\
= & \sqrt{n}\left(p_{k}\left(x_{i}, v_{1}^{*}\right)^{\prime} \widehat{\vartheta}_{1}-p_{k}\left(x_{i}, v_{1}^{*}\right)^{\prime} \vartheta_{1}\right)+\sqrt{n}\left(p_{k}\left(x_{i}, \widehat{v}_{1 n}\right)^{\prime} \widehat{\vartheta}_{1}-p_{k}\left(x_{i}, v_{1}^{*}\right)^{\prime} \widehat{\vartheta}_{1}\right)+O\left(n^{1 / 2} k^{-r}\right),
\end{aligned}
$$

and $\sqrt{n}\left(p_{k}\left(x_{i}, v_{1}^{*}\right)^{\prime} \widehat{\vartheta}_{1}-p_{k}\left(x_{i}, v_{1}^{*}\right)^{\prime} \vartheta_{1}\right)$ satisfies Condition $\mathrm{C} 2$ by assumption. We are left to show that the second term in equation (B.6) converges to zero in probability as $n \rightarrow \infty$. Recall that $\widehat{v}_{1 n}$ is a minimizer of $p_{k}\left(x_{i}, v_{i}\right)^{\prime} \widehat{\vartheta}_{1}$ and $v_{1}^{*}$ is the unique minimizer of $\theta_{1}(x, v, \alpha)$, and therefore

$$
\begin{aligned}
0 \geq & \sqrt{n}\left(p_{k}\left(x_{i}, \widehat{v}_{1 n}\right)^{\prime} \widehat{\vartheta}_{1}-p_{k}\left(x_{i}, v_{1}^{*}\right)^{\prime} \widehat{\vartheta}_{1}\right) \\
= & \sqrt{n}\left(p_{k}\left(x_{i}, \widehat{v}_{1 n}\right)^{\prime} \widehat{\vartheta}_{1}-p_{k}\left(x_{i}, \widehat{v}_{1 n}\right)^{\prime} \vartheta_{1}\right)+\sqrt{n}\left(p_{k}\left(x_{i}, \widehat{v}_{1 n}\right)^{\prime} \vartheta_{1}-p_{k}\left(x_{i}, v_{1}^{*}\right)^{\prime} \vartheta_{1}\right) \\
& +\sqrt{n}\left(p_{k}\left(x_{i}, v_{1}^{*}\right)^{\prime} \vartheta_{1}-p_{k}\left(x_{i}, v_{1}^{*}\right)^{\prime} \widehat{\vartheta}_{1}\right) \\
= & \sqrt{n}\left(p_{k}\left(x_{i}, \widehat{v}_{1 n}\right)^{\prime} \widehat{\vartheta}_{1}-p_{k}\left(x_{i}, \widehat{v}_{1 n}\right)^{\prime} \vartheta_{1}\right)+\sqrt{n}\left(\theta_{1}\left(x, \widehat{v}_{1 n}, \alpha\right)-\theta_{1}\left(x, v_{1}^{*}, \alpha\right)\right)+O\left(n^{1 / 2} k^{-r}\right) \\
& -\sqrt{n}\left(p_{k}\left(x_{i}, v_{1}^{*}\right)^{\prime} \widehat{\vartheta}_{1}-p_{k}\left(x_{i}, v_{1}^{*}\right)^{\prime} \vartheta_{1}\right) \\
\geq & \sqrt{n}\left(p_{k}\left(x_{i}, \widehat{v}_{1 n}\right)-p_{k}\left(x_{i}, v_{1}^{*}\right)\right)^{\prime}\left(\widehat{\vartheta}_{1}-\vartheta_{1}\right)+O\left(n^{1 / 2} k^{-r}\right) \\
= & o_{p}(1) .
\end{aligned}
$$

To establish the last equality in (B.8), let $\mathcal{V}=\left\{v^{1}, \ldots, v^{J}\right\}$ and notice that

$$
p_{k}\left(x_{i}, \tilde{v}_{1}\right)^{\prime} \vartheta=\sum_{j=1}^{J} p_{k}\left(x_{i}, v_{j}\right)^{\prime} \vartheta 1\left(\tilde{v}_{1}=v_{j}\right), \quad \vartheta=\widehat{\vartheta}_{1}, \vartheta_{1}, \text { and } \tilde{v}_{1}=\widehat{v}_{1 n}, v_{1}^{*} \text {. }
$$

\footnotetext{
${ }^{14}$ As stated in the examples, when using either polynomials or splines, this condition is met for mean regression with $r=\frac{s}{d}$, for quantile regression with $r=\frac{s-a d}{d}$, and for distribution regression with $r=\frac{s-2 a d}{2 d}$, where $s$ is the smoothness of $\theta_{\ell}, d$ is the dimension of $x$, and $a=1$ for polynomials or $a=1 / 2$ for splines. Notice that $n^{1 / 2} k^{-r} \rightarrow 0$ for our motivating examples under the conditions derived in Section 4.2
} 
Hence, $\sqrt{n}\left(p_{k}\left(x_{i}, \widehat{v}_{1 n}\right)-p_{k}\left(x_{i}, v_{1}^{*}\right)\right)^{\prime}\left(\widehat{\vartheta}_{1}-\vartheta_{1}\right)=\sqrt{n} \sum_{j=1}^{J} p_{k}\left(x_{i}, v_{j}\right)^{\prime}\left(\widehat{\vartheta}_{1}-\vartheta_{1}\right)\left[1\left(\widehat{v}_{1 n}=v_{j}\right)-1\left(v_{1}^{*}=v_{j}\right)\right]$.

Standard results give

$$
\sqrt{n} \sum_{j=1}^{J} p_{k}\left(x_{i}, v_{j}\right)^{\prime}\left(\widehat{\vartheta}_{1}-\vartheta_{1}\right)=O_{p}(\sqrt{k})
$$

Next, observe that

$$
\begin{aligned}
& \mathrm{P}\left\{\widehat{v}_{1 n}=v_{1}^{*}\right\}=\mathrm{P}\left(p_{k}\left(x_{i}, v_{1}^{*}\right)^{\prime} \widehat{\vartheta}_{1} \leq p_{k}\left(x_{i}, v^{j}\right)^{\prime} \widehat{\vartheta}_{1} \quad \forall v^{j} \in \mathcal{V}: v^{j} \neq v^{*}\right)
\end{aligned}
$$

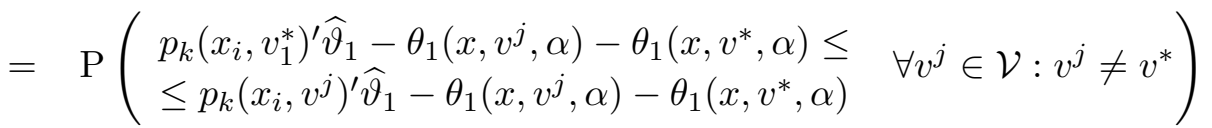

$$
\begin{aligned}
& =\mathrm{P}\left(\begin{array}{l}
\left(p_{k}\left(x_{i}, v_{1}^{*}\right)^{\prime} \widehat{\vartheta}_{1}-\theta_{1}\left(x, v^{*}, \alpha\right)\right)-\left(p_{k}\left(x_{i}, v^{j}\right)^{\prime} \widehat{\vartheta}_{1}-\theta_{1}\left(x, v^{j}, \alpha\right)\right) \leq \forall v^{j} \in \mathcal{V}: v^{j} \neq v^{*} \\
\leq \theta_{1}\left(x, v^{j}, \alpha\right)-\theta_{1}\left(x, v^{*}, \alpha\right)
\end{array}\right.
\end{aligned}
$$

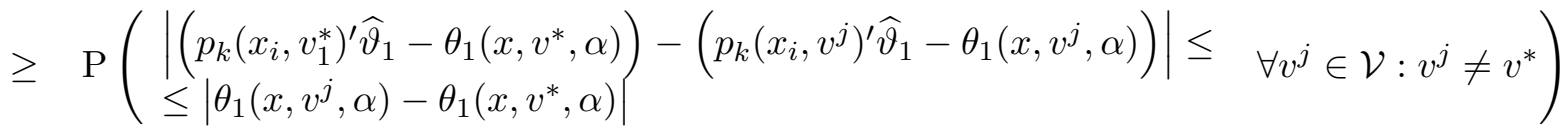

$$
\begin{aligned}
& \geq \mathrm{P}\left(\begin{array}{l}
\left.\left|p_{k}\left(x_{i}, v_{1}^{*}\right)^{\prime} \widehat{\vartheta}_{1}-\theta_{1}\left(x, v^{*}, \alpha\right)\right|+\left|p_{k}\left(x_{i}, v^{j}\right)^{\prime} \widehat{\vartheta}_{1}-\theta_{1}\left(x, v^{j}, \alpha\right)\right| \leq \quad \forall v^{j} \in \mathcal{V}: v^{j} \neq v^{*}\right) \\
\leq\left|\theta_{1}\left(x, v^{j}, \alpha\right)-\theta_{1}\left(x, v^{*}, \alpha\right)\right|
\end{array}\right. \\
& \gtrsim p \quad 1-\max \left\{\left(\frac{k}{n}\right)^{1 / 2}, k^{-r}\right\} \text {, }
\end{aligned}
$$

where the first inequality follows from the assumption that $\theta_{1}$ is uniquely minimized at $v^{*}$ and the last line follows from $(\mathrm{B} .5)$ and $(\mathrm{B} .9)$. This yields

$$
\left[1\left(\widehat{v}_{1 n}=v_{j}\right)-1\left(v_{1}^{*}=v_{j}\right)\right]=O_{p}\left(\max \left\{\left(\frac{k}{n}\right)^{1 / 2}, k^{-r}\right\}\right)
$$

and hence as long as $k \propto n^{\gamma}$ for any $\gamma<\frac{1}{2}$, the claim follows. Note that this condition is consistent with the requirements on the growth of $k$ that we obtained in verifying our assumptions for the motivating examples in Section 4.2 .

B.4. Conservative Inference with Discrete Covariates. Let $\Theta(x, \alpha)=\left[\theta_{0}(x, \alpha), \theta_{1}(x, \alpha)\right]$, and to simplify notation suppress the dependence of $\Theta$ and $\theta_{\ell}$ on $(x, \alpha)$ and let the instruments coincide with $x=\left[x_{1} x_{2}\right]^{\prime}$, with $x_{1}=1$ and $x_{2} \in \mathbb{R}^{d-1}$. Let $\Sigma=\mathrm{E}\left(x x^{\prime}\right)^{-1}$, $z=x+\sigma[0 \eta]^{\prime}$, with $\eta \sim N(0, I)$ and independent of $x$ and $\theta_{\ell}, \ell=0,1$, where $I$ denotes the identity matrix. Note that $\mathrm{E}\left(x x^{\prime}\right)=\mathrm{E}\left(z x^{\prime}\right)$, and define

$$
B=\Sigma \mathbf{E}(x \Theta), \quad \tilde{B}=\Sigma \mathbf{E}(z \Theta),
$$


where $\mathbf{E}(\cdot)$ denotes the Aumann expectation of the random set in parenthesis, see Molchanov (2005, Chapter 2). Denote by $\widehat{\tilde{B}}$ the estimator of $\tilde{B}$ (the unique convex set corresponding to the estimated support function) and by $\mathcal{B}_{\widehat{c}_{n(1-\tau)}}$ a ball in $\mathbb{R}^{d}$ centered at zero and with radius $\widehat{c}_{n(1-\tau)}$, with $\widehat{c}_{n(1-\tau)}$ the Bayesian bootstrap estimate of the $1-\tau$ quantile of $f\left(\mathbb{G}\left[h_{k}(t)\right]\right)$, with $f(s(t))=\sup _{t \in T}\{-s(t)\}_{+}$, see Section 4.3. Following arguments in BM (Section 2.3), one can construct a (convex) confidence set $C S_{n}$ such that $\sup _{\alpha \in \mathcal{A}}\left(\sigma_{C S_{n}}(q)-\sigma_{\widehat{\tilde{B}}}(q, \alpha)\right)=\widehat{c}_{n(1-\tau)}$ for all $q \in \mathcal{S}^{d-1}$, where $\sigma_{A}(\cdot)$ denotes the support function of the set $A$. It then follows that

$$
\lim _{n \rightarrow \infty} \mathrm{P}\left(\sup _{q, \alpha \in \mathcal{S}^{d-1} \times \mathcal{A}}\left|\sigma_{\tilde{B}}(q, \alpha)-\sigma_{C S_{n}}(q)\right|_{+}=0\right)=1-\tau .
$$

Lemma 8. For a given $\delta>0$, one can jitter $x$ via $z=x+\sigma_{\delta}[0 \eta]^{\prime}$, so as to obtain a set $\tilde{B}$ such that $\sup _{\alpha \in \mathcal{A}} \rho_{H}(\tilde{B}, B) \leq \delta$ and

$$
1-\gamma(\delta) \geq \lim _{n \rightarrow \infty} \mathrm{P}\left(\sup _{q, \alpha \in \mathcal{S}^{d-1} \times \mathcal{A}}\left|\sigma_{B}(q, \alpha)-\left(\sigma_{C S_{n}}(q)+\delta\right)\right|_{+}=0\right) \geq 1-\tau,
$$

where $\gamma(\delta)=\mathrm{P}\left(\sup _{t \in T}\left\{-\mathbb{G}\left[h_{k}(t)\right]\right\}_{+}>c_{n(1-\tau)}+2 \delta\right)$.

Proof. Observe that $\rho_{H}(\tilde{B}, B)=\rho_{H}(\Sigma \mathbf{E}(z \Theta), \Sigma \mathbf{E}(x \Theta))$. By the properties of the Aumann expectation (see, e.g., Molchanov (2005, Theorem 2.1.17)),

$$
\rho_{H}(\Sigma \mathbf{E}(z \Theta), \Sigma \mathbf{E}(x \Theta)) \leq \mathrm{E}\left[\rho_{H}(\Sigma(z \Theta), \Sigma(x \Theta))\right] .
$$

In turn,

$$
\begin{aligned}
& \sup _{\alpha \in \mathcal{A}} \mathrm{E}\left[\rho_{H}(\Sigma(z \Theta), \Sigma(x \Theta))\right] \\
&= \sup _{\alpha \in \mathcal{A}} \mathrm{E}\left[\sup _{v=\Sigma^{\prime} q:\|v\|=1}\left|\sup _{\theta \in \Theta}\left(v_{1}+z_{2} v_{2}\right) \tilde{\theta}-\sup _{\theta \in \Theta}\left(v_{1}+x_{2} v_{2}\right) \theta\right|\right] \\
&= \sup _{\alpha \in \mathcal{A}} \mathrm{E}\left[\sup _{v=\Sigma^{\prime} q:\|v\|=1} \mid\left(v_{1}+x_{2} v_{2}+\sigma \eta v_{2}\right)\left(\theta_{0} 1\left(v_{1}+x_{2} v_{2}+\sigma \eta v_{2}<0\right)+\theta_{1} 1\left(v_{1}+x_{2} v_{2}+\sigma \eta v_{2}>0\right)\right)\right. \\
&\left.-\left(v_{1}+x_{2} v_{2}\right)\left(\theta_{0} 1\left(v_{1}+x_{2} v_{2}<0\right)+\theta_{1} 1\left(v_{1}+x_{2} v_{2}>0\right)\right) \mid\right] \\
& \leq \sup _{\alpha \in \mathcal{A}} \mathrm{E}\left[\sup _{v=\Sigma^{\prime} q:\|v\|=1}\left|\sigma \eta v_{2}\left(\theta_{0} 1\left(v_{1}+x_{2} v_{2}+\sigma \eta v_{2}<0\right)+\theta_{1} 1\left(v_{1}+x_{2} v_{2}+\sigma \eta v_{2}>0\right)\right)\right|\right] \\
&+\sup _{\alpha \in \mathcal{A}} \mathrm{E}\left[\sup _{v=\Sigma^{\prime} q:\|v\|=1}\left|\left(v_{1}+x_{2} v_{2}\right)\left(\theta_{1}-\theta_{0}\right)\left(1\left(0<-\left(v_{1}+x_{2} v_{2}\right)<\sigma \eta v_{2}\right)-1\left(0<v_{1}+x_{2} v_{2}<-\sigma \eta v_{2}\right)\right)\right|\right] \\
& \leq \sigma \mathrm{E}|\eta|\left(\sup _{\alpha \in \mathcal{A}} \mathrm{E}\left|\theta_{0}(x, \alpha)\right|+\sup _{\alpha \in \mathcal{A}} \mathrm{E}\left|\theta_{1}(x, \alpha)\right|+\sup _{\alpha \in \mathcal{A}} \mathrm{E}\left|\theta_{1}(x, \alpha)-\theta_{0}(x, \alpha)\right|\right) \\
& \text { Hence, we } \operatorname{can} \operatorname{choose} \sigma_{\delta}=\frac{\delta}{\mathrm{E}|\eta|\left(\sup _{\alpha \in \mathcal{A}} \mathrm{E}\left|\theta_{0}(x, \alpha)\right|+\sup _{\alpha \in \mathcal{A}} \mathrm{E}\left|\theta_{1}(x, \alpha)\right|+\sup _{\alpha \in \mathcal{A}} \mathrm{E}\left|\theta_{1}(x, \alpha)-\theta_{0}(x, \alpha)\right|\right)}
\end{aligned}
$$


Now observe that because $\sup _{\alpha \in \mathcal{A}} \rho_{H}(\tilde{B}, B) \leq \delta$, we have $B(\alpha) \subseteq \tilde{B}(\alpha) \oplus \mathcal{B}_{\delta}$ for all $\alpha \in \mathcal{A}$, where " $\oplus$ " denotes Minkowski set summation and $\mathcal{B}_{\delta}$ is a ball of radius $\delta$ centered at the origin. Therefore

$$
\begin{aligned}
\sup _{\alpha \in \mathcal{A}}\left(\sigma_{\tilde{B}}(q, \alpha)-\sigma_{C S_{n}}(q)\right) & \leq 0 \forall q \in \mathcal{S}^{d-1} \\
& \Longrightarrow \sup _{\alpha \in \mathcal{A}}\left(\sigma_{B}(q, \alpha)-\left(\sigma_{C S_{n}}(q)+\delta\right)\right) \leq 0 \forall q \in \mathcal{S}^{d-1},
\end{aligned}
$$

from which the second inequality in $\mathrm{B} .10$ follows. Notice also that $\tilde{B}(\alpha) \subseteq B(\alpha) \oplus \mathcal{B}_{\delta}$ for all $\alpha \in \mathcal{A}$, and therefore

$$
\begin{aligned}
\sup _{\alpha \in \mathcal{A}}\left(\sigma_{B}(q, \alpha)-\left(\sigma_{C S_{n}}(q)+\delta\right)\right) & \leq 0 \forall q \in \mathcal{S}^{d-1} \\
& \Longrightarrow \sup _{\alpha \in \mathcal{A}}\left(\sigma_{\tilde{B}}(q, \alpha)-\left(\sigma_{C S_{n}}(q)+2 \delta\right)\right) \leq 0 \forall q \in \mathcal{S}^{d-1},
\end{aligned}
$$

from which the first inequality in $\mathrm{B} .10$ follows. Because $\delta>0$ is chosen by the researcher, inference is arbitrarily slightly conservative. Note that a similar argument applies if one uses a Kolmogorov statistic rather than a directed Kolmogorov statistic. Moreover, the Hausdorff distance among convex compact sets is larger than the $L_{p}$ distance among them (see, e.g., Vitale (1985, Theorem 1)), and therefore a similar conclusion applies for CramerVon-Mises statistics.

B.5. Lemmas on Entropy Bounds. We collect frequently used facts in the following lemma.

Lemma 9. Let $Q$ be any probability measure whose support concentrates on a finite set.

(1) Let $\mathcal{F}$ be a measurable $V C$ class with a finite $V C$ index $k$ or any other class whose entropy is bounded above by that of such a VC class, then its entropy obeys

$$
\log N\left(\epsilon\|F\|_{Q, 2}, \mathcal{F}, L^{2}(Q)\right) \lesssim 1+k \log (1 / \epsilon)
$$

Examples include e.g., linear functions $\mathcal{F}=\left\{\alpha^{\prime} w_{i}, \alpha \in \mathbb{R}^{k},\|\alpha\| \leq C\right\}$ and their indicators $\mathcal{F}=\left\{1\left\{\alpha^{\prime} w_{i}>0\right\}, \alpha \in \mathbb{R}^{k},\|\alpha\| \leq C\right\}$.

(2) Entropies obey the following rules for sets created by addition, multiplication, and unions of measurable function sets $\mathcal{F}$ and $\mathcal{F}^{\prime}$ :

$$
\begin{aligned}
& \log N\left(\epsilon\left\|F+F^{\prime}\right\|_{Q, 2}, \mathcal{F}+\mathcal{F}^{\prime}, L^{2}(Q)\right) \leq B \\
& \log N\left(\epsilon\left\|F \cdot F^{\prime}\right\|_{Q, 2}, \mathcal{F} \cdot \mathcal{F}^{\prime}, L^{2}(Q)\right) \leq B \\
& \log N\left(\epsilon\left\|F \vee F^{\prime}\right\|_{Q, 2}, \mathcal{F} \cup \mathcal{F}^{\prime}, L^{2}(Q)\right) \leq B \\
& B=\log N\left(\frac{\epsilon}{2}\|F\|_{Q, 2}, \mathcal{F}, L^{2}(Q)\right)+\log N\left(\frac{\epsilon}{2}\left\|F^{\prime}\right\|_{Q, 2}, \mathcal{F}^{\prime}, L^{2}(Q)\right) .
\end{aligned}
$$

(3) Entropies are preserved by multiplying a measurable function class $\mathcal{F}$ with a random variable $g_{i}$ :

$$
\log N\left(\epsilon\||g| F\|_{Q, 2}, g \mathcal{F}, L^{2}(Q)\right) \lesssim \log N\left(\epsilon / 2\|F\|_{Q, 2}, \mathcal{F}, L^{2}(Q)\right)
$$


(4) Entropies are preserved by integration or taking expectation: for $f^{*}(x):=\int f(x, y) d \mu(y)$ where $\mu$ is some probability measure,

$$
\log N\left(\epsilon\|F\|_{Q, 2}, \mathcal{F}^{*}, L^{2}(Q)\right) \leq \log N\left(\epsilon\|F\|_{Q, 2}, \mathcal{F}, L^{2}(Q)\right)
$$

Proof. For the proof of (1)-(3) see e.g., Andrews (1994). For the proof of (4), see e.g., Ghosal, Sen, and van der Vaart (2000, Lemma A2).

Next consider function classes and their envelops

$$
\begin{aligned}
\mathcal{H}_{1} & =\left\{q^{\prime} \Sigma \mathrm{E}\left[z_{i} p_{i}^{\prime} 1\left\{q^{\prime} \Sigma z_{i}<0\right\}\right] J_{0}^{-1}(\alpha) p_{i} \varphi_{i 0}(\alpha), t \in T\right\}, \quad H_{1} \lesssim\left\|z_{i}\right\| \xi_{k} F_{1} \\
\mathcal{H}_{2} & =\left\{q^{\prime} \Sigma \mathrm{E}\left[z_{i} p_{i}^{\prime} 1\left\{q^{\prime} \Sigma z_{i}>0\right\}\right] J_{1}^{-1}(\alpha) p_{i} \varphi_{i 1}(\alpha), t \in T\right\}, \quad H_{2} \lesssim\left\|z_{i}\right\| \xi_{k} F_{1} \\
\mathcal{H}_{3} & =\left\{q^{\prime} \Sigma x_{i} z_{i}^{\prime} \Sigma \mathrm{E}\left[z_{i} w_{i, q^{\prime} \Sigma}(\alpha)\right], t \in T\right\}, \quad H_{3} \lesssim\left\|x_{i}\right\|\left\|z_{i}\right\| \\
\mathcal{H}_{4} & =\left\{q^{\prime} \Sigma z_{i} w_{i, q^{\prime} \Sigma}(\alpha), t \in T\right\}, \quad H_{4} \lesssim\left\|z_{i}\right\| F_{2} \\
\mathcal{F}_{3} & =\left\{\bar{\mu}^{\prime} J^{-1}(\alpha) p_{i} \varphi_{i}(\alpha), \alpha \in \mathcal{A}\right\}, \quad F_{3} \lesssim \xi_{k} F_{1},
\end{aligned}
$$

where $\bar{\mu}^{\prime}$ is defined in equation $(\overline{B .2})$.

Lemma 10. 1. (a) The following bounds on the empirical entropy apply

$$
\begin{aligned}
& \log N\left(\epsilon\left\|H_{1}\right\|_{\mathbb{P}_{n}, 2}, \mathcal{H}_{1}, L^{2}\left(\mathbb{P}_{n}\right)\right) \lesssim_{\mathrm{P}} \log n+\log (1 / \epsilon) \\
& \log N\left(\epsilon\left\|H_{2}\right\|_{\mathbb{P}_{n}, 2}, \mathcal{H}_{2}, L^{2}\left(\mathbb{P}_{n}\right)\right) \lesssim_{\mathrm{P}} \log n+\log (1 / \epsilon) \\
& \log N\left(\epsilon\left\|F_{3}\right\|_{\mathbb{P}_{n}, 2}, \mathcal{F}_{3}, L^{2}\left(\mathbb{P}_{n}\right)\right) \lesssim_{\mathrm{P}} \log n+\log (1 / \epsilon)
\end{aligned}
$$

(b) Moreover similar bounds apply to function classes $g_{i}\left(\mathcal{H}_{l}^{o}-\mathcal{H}_{l}^{o}\right)$ with the envelopes given by $\left|g_{i}\right| 4 H \ell$, where $g_{i}$ is a random variable.

2. (a) The following bounds on the uniform entropy apply

$$
\begin{aligned}
& \sup _{Q} \log N\left(\epsilon\left\|H_{1}\right\|_{Q, 2}, \mathcal{H}_{1}, L^{2}(Q)\right) \lesssim k \log (1 / \epsilon) \\
& \sup _{Q} \log N\left(\epsilon\left\|H_{2}\right\|_{Q, 2}, \mathcal{H}_{2}, L^{2}(Q)\right) \lesssim k \log (1 / \epsilon) \\
& \sup _{Q} \log N\left(\epsilon\left\|F_{3}\right\|_{Q, 2}, \mathcal{F}_{3}, L^{2}(Q)\right) \lesssim k \log (1 / \epsilon) \\
& \sup _{Q} \log N\left(\epsilon\left\|H_{3}\right\|_{Q, 2}, \mathcal{H}_{3}, L^{2}(Q)\right) \lesssim \log (1 / \epsilon) \\
& \sup _{Q} \log N\left(\epsilon\left\|H_{4}\right\|_{Q, 2}, \mathcal{H}_{4}, L^{2}(Q)\right) \lesssim \log (1 / \epsilon) .
\end{aligned}
$$

(b) Moreover similar bounds apply to function classes $g_{i}\left(\mathcal{H}_{l}^{o}-\mathcal{H}_{l}^{o}\right)$ with the envelopes given by $\left|g_{i}\right| 4 H \ell$, where $g_{i}$ is a random variable.

Proof. Part 1 (a). Case of $\mathcal{H}_{1}$ and $\mathcal{H}_{2}$. We shall detail the proof for this case, while providing shorter arguments for others, as they are simpler or similar.

Note that $\mathcal{H}_{1} \subseteq \mathcal{M}_{1} \cdot \mathcal{M}_{2} \cdot \mathcal{F}_{1}$, where $\mathcal{M}_{1}=\left\{q^{\prime} \Sigma z_{i}, q \in \mathcal{S}^{d-1}\right\}$ with envelope $M_{1}=\left\|z_{i}\right\|$ is $\mathrm{VC}$ with index $\operatorname{dim}\left(z_{i}\right)+\operatorname{dim}\left(x_{i}\right)$, and $\mathcal{M}_{2}=\left\{\gamma(q) J_{0}^{-1}(\alpha) p_{i},(q, \alpha) \in \mathcal{S}^{d-1} \times \mathcal{A}\right\}$ with envelope $M_{2} \lesssim\left\|\xi_{k}\right\|, \mathcal{F}_{1}=\left\{\varphi_{i 0}(\alpha), \alpha \in \mathcal{A}\right\}$ with envelope $F_{1}$, where $\gamma(q)$ is uniformly 
Holder in $q \in \mathcal{S}^{d-1}$ by Lemma 3 . Elementary bounds yield

$$
\begin{aligned}
& \left\|m_{2}(t)-m_{2}(\tilde{t})\right\|_{\mathbb{P}_{n}, 2} \leq L_{1 n}\|\alpha-\tilde{\alpha}\|+L_{2 n}\|q-\tilde{q}\|, \\
& L_{1 n} \lesssim \sup _{\alpha \in \mathcal{A}}\left\|J^{-1}(\alpha)\right\|\left\|\xi_{k}\right\| \quad L_{2 n} \lesssim\left\|\mathbb{E}_{n}\left[p_{i} p_{i}^{\prime}\right]\right\|, \\
& \log L_{1 n} \lesssim_{\mathrm{P}} \log n \text { and } \log L_{2 n} \lesssim_{\mathrm{P}} 1 .
\end{aligned}
$$

Note that $\log \xi_{k} \lesssim \log n$ by assumption, $\sup _{\alpha \in \mathcal{A}}\left\|J^{-1}(\alpha)\right\| \lesssim 1$ by assumption, $\left\|\mathbb{E}_{n}\left[p_{i} p_{i}^{\prime}\right]\right\| \lesssim \mathrm{P}$ 1 by Lemma 11. The sets $\mathcal{S}^{d-1}$ and $\mathcal{A}$ are compact subsets of Euclidian space of fixed dimension, and so can be covered by a constant times $1 / \epsilon^{c}$ balls of radius $\epsilon$ for some constant $c>0$. Therefore, we can conclude

$$
\log N\left(\epsilon\left\|M_{2}\right\|_{\mathbb{P}_{n}, 2}, \mathcal{M}_{2}, L_{2}\left(\mathbb{P}_{n}\right)\right) \lesssim_{\mathrm{P}} \log n+\log (1 / \epsilon) .
$$

Repeated application of Lemma 9 yields the conclusion, given the assumption on the function class $\mathcal{F}_{1}$. The case for $\mathcal{H}_{2}$ is very similar.

Case of $\mathcal{F}_{3}$. Note that $\mathcal{F}_{3} \subset \mathcal{M}_{2} \cdot \mathcal{F}_{1}$ and $\|\bar{\mu}\|=o_{\mathrm{P}}(1)$ by Step 4 in the proof of Lemma 1. Repeated application of Lemma 9 yields the conclusion, given the assumption on the function class $\mathcal{F}_{1}$.

Part 1 (b). Note that $\mathcal{H}^{o}=\mathcal{H}-\mathrm{E}\left[\mathcal{H}^{o}\right]$, so it is created by integration and summation. Hence repeated application of Lemma 9 yields the conclusion.

Part 2. (a) Case of $\mathcal{H}_{1}, \mathcal{H}_{2}$, and $\mathcal{F}_{3}$. Note that all of these classes are subsets of $\left\{\mu^{\prime} p_{i},\|\mu\| \leq C\right\} \cdot \mathcal{F}_{1}$ with envelope $\xi_{k} F_{1}$. The claim follows from repeated application of Lemma 9 .

Case of $\mathcal{H}_{3}$. Note that $\mathcal{H}_{3} \subset\left\{q^{\prime} \Sigma x_{i} z_{i}^{\prime} \mu,\|\mu\| \leq C\right\}$ with envelope $\left\|x_{i}\right\|\left\|z_{i}\right\|$. The claim follows from repeated application of Lemma 9.

Case of $\mathcal{H}_{4}$. Note that $\mathcal{H}_{4}$ is a subset of a function class created from taking the class $\mathcal{F}_{2}$ multiplying it with indicator function class $1\left\{q^{\prime} \Sigma z_{i}>0, q \in \mathcal{S}^{d-1}\right\}$ and with function class $\left\{q^{\prime} \Sigma z_{i}, q \in \mathcal{S}^{d-1}\right\}$ and then adding the resulting class to itself. The claim follows from repeated application of Lemma 9 .

Part 2 (b). Note that $\mathcal{H}^{o}=\mathcal{H}-\mathrm{E}\left[\mathcal{H}^{o}\right]$, so it is created by integration and summation. Hence repeated application of Lemma 9 yields the conclusion.

B.6. Auxiliary Maximal and Random Matrix Inequalities. We repeatedly use the following matrix LLN.

Lemma 11 (Matrix LLN). Let $Q_{1}, \ldots, Q_{n}$ be i.i.d. symmetric non-negative matrices such that $Q=\mathrm{E} Q_{i}$ and $\left\|Q_{i}\right\| \leq M$, then for $\widehat{Q}=\mathbb{E}_{n} Q_{i}$

$$
\mathrm{E}\|\widehat{Q}-Q\| \lesssim \sqrt{\frac{M(1+\|Q\|) \log k}{n}} .
$$

In particular, if $Q_{i}=p_{i} p_{i}^{\prime}$, with $\left\|p_{i}\right\| \leq \xi_{k}$, then

$$
\mathrm{E}\|\widehat{Q}-Q\| \lesssim \sqrt{\frac{\xi_{k}^{2}(1+\|Q\|) \log k}{n}} .
$$


Proof. This is a variant of a result from Rudelson (1999). By the symmetrization lemma,

$$
\Delta:=\mathrm{E}\|\widehat{Q}-Q\| \leq 2 \mathrm{EE}_{\epsilon}\left\|\mathbb{E}_{n}\left[\epsilon_{i} Q_{i}\right]\right\|
$$

where $\epsilon_{i}$ are Rademacher random variables. The Khintchine inequality for matrices, which was shown by Rudelson (1999) to follow from the results of Lust-Piquard and Pisier (1991), states that

$$
\mathrm{E}_{\epsilon}\left\|\mathbb{E}_{n}\left[\epsilon_{i} Q_{i}\right]\right\| \lesssim \sqrt{\frac{\log k}{n}}\left\|\left(\mathbb{E}_{n}\left[Q_{i}^{2}\right]\right)^{1 / 2}\right\|
$$

Since (remember that $\|\cdot\|$ is the operator norm)

$$
\mathrm{E}\left\|\left(\mathbb{E}_{n}\left[Q_{i}^{2}\right]\right)^{1 / 2}\right\|=\mathrm{E}\left\|\left(\mathbb{E}_{n}\left[Q_{i}^{2}\right]\right)\right\|^{1 / 2} \leq\left[M \mathrm{E}\left\|\mathbb{E}_{n} Q_{i}\right\|\right]^{1 / 2}
$$

and

one has

$$
\left\|\mathbb{E}_{n} Q_{i}\right\| \leq \Delta+\|Q\|
$$

$$
\Delta \leq 2 \sqrt{\frac{M \log k}{n}}[\Delta+\|Q\|]^{1 / 2}
$$

Solving for $\Delta$ gives

$$
\Delta \leq \sqrt{\frac{4 M\|Q\| \log k}{n}+\left(\frac{M \log k}{n}\right)^{2}}+\frac{M \log k}{n},
$$

which implies the result stated in the lemma if $\frac{M \log k}{n}<1$.

We also use the following maximal inequality.

Lemma 12. Consider a separable empirical process $\mathbb{G}_{n}(f)=n^{-1 / 2} \sum_{i=1}^{n}\left\{f\left(Z_{i}\right)-\mathrm{E}\left[f\left(Z_{i}\right)\right]\right\}$, where $Z_{1}, \ldots, Z_{n}$ is an underlying independent data sequence on the space $(\Omega, \mathcal{G}, \mathrm{P})$, defined over the function class $\mathcal{F}$, with an envelope function $F \geq 1$ such that $\log \left[\max _{i \leq n}\|F\|\right] \lesssim_{\mathrm{P}}$ $\log n$ and

$$
\log N\left(\varepsilon\|F\|_{\mathbb{P}_{n}, 2}, \mathcal{F}, L_{2}\left(\mathbb{P}_{n}\right)\right) \leq v m \log (\kappa / \epsilon), \quad 0<\epsilon<1,
$$

with some constants $0<\log \kappa \lesssim \log n, m$ potentially depending on $n$, and $1<v \lesssim 1$. For any $\delta \in(0,1)$, there is a constant $K_{\delta}$ large enough, such that for $n$ sufficiently large

$$
\mathrm{P}\left\{\sup _{f \in \mathcal{F}}\left|\mathbb{G}_{n}(f)\right| \leq K_{\delta} \sqrt{m \log n} \max \left\{\sup _{i \leq n, f \in \mathcal{F}}\left\|f\left(Z_{i}\right)\right\|_{\mathrm{P}, 2}, \sup _{f \in \mathcal{F}}\|f\|_{\mathbb{P}_{n}, 2}\right\}\right\} \geq 1-\delta .
$$

Proof. This is a restatement of Lemma 19 from Belloni and Chernozhukov (2009b). 


\section{Appendix C. Additional Results for the Empirical Application}

\section{Figure C.1. Quantile bounds for singles}

1975-1979

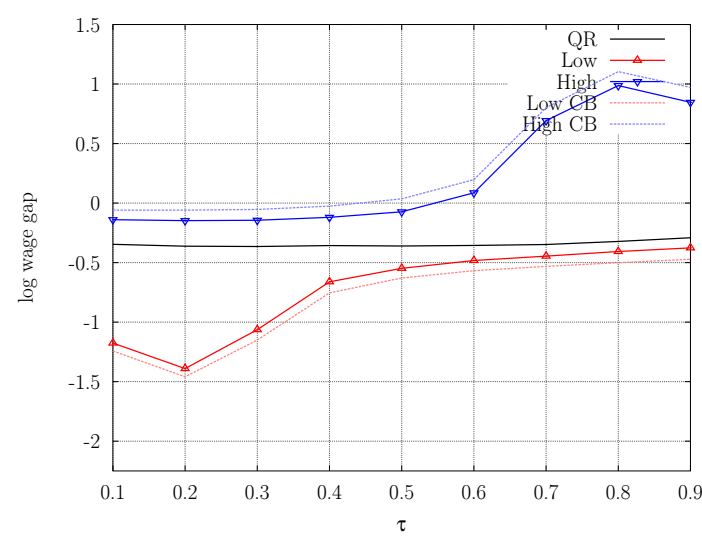

1995-1999

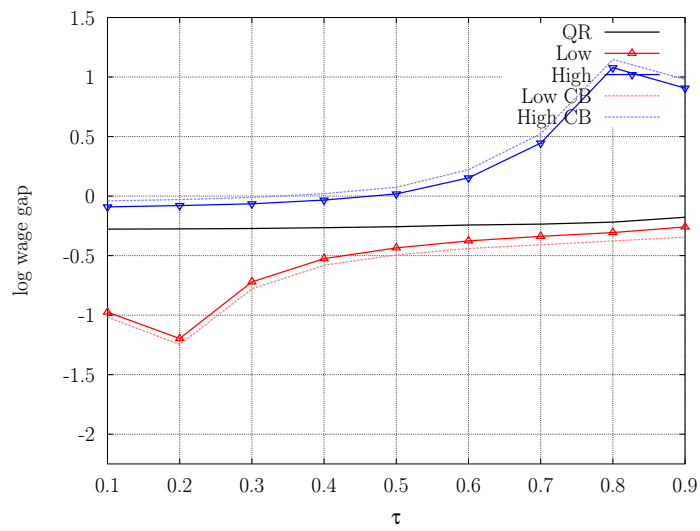

Estimated quantile gender wage gap (female - male) conditional on being single with average characteristics. The solid black line shows the quantile gender wage gap when selection is ignored. The blue and red lines with upward and downward pointing triangles show upper and lower bounds that account for employment selection for females. The dashed lines represent a uniform $90 \%$ confidence region for the bounds.

Figure C.2. Quantile bounds for $\geq 16$ years of education 1975-1979 1995-1999
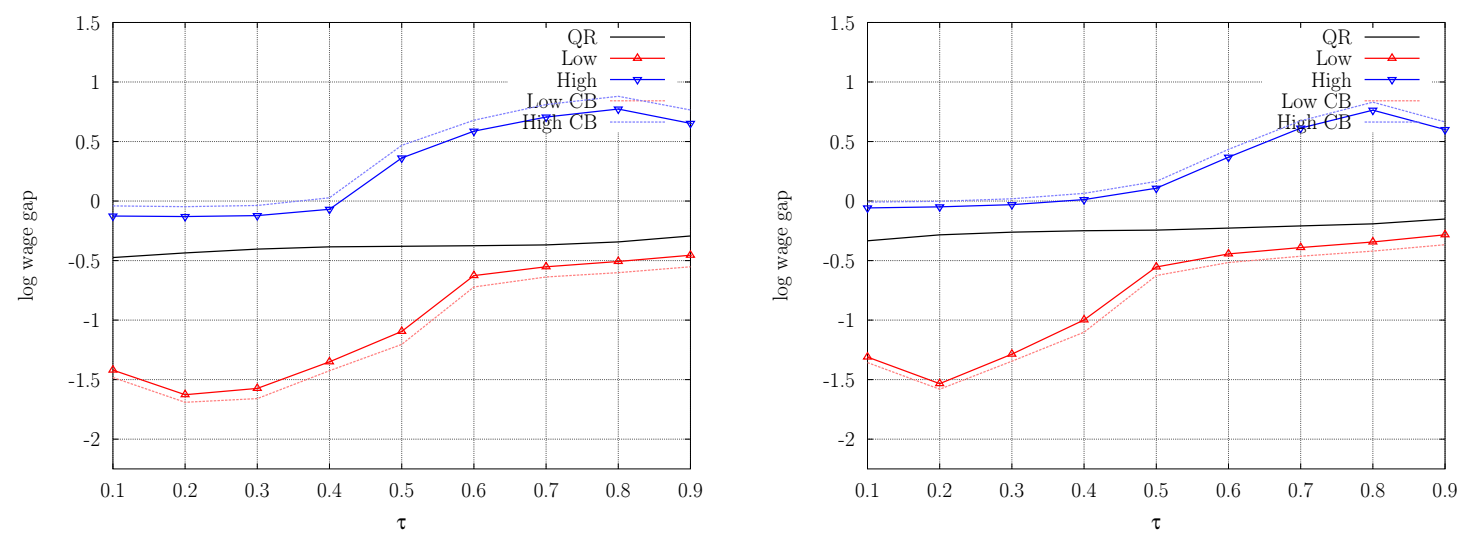

Estimated quantile gender wage gap (female - male) conditional on having at least 16 years of education with average characteristics. The solid black line shows the quantile gender wage gap when selection is ignored. The blue and red lines with upward and downward pointing triangles show upper and lower bounds that account for employment selection for females. The dashed lines represent a uniform $90 \%$ confidence region for the bounds. 
FIGURE C.3. Quantile bounds for $\leq 12$ years of education

1975-1979

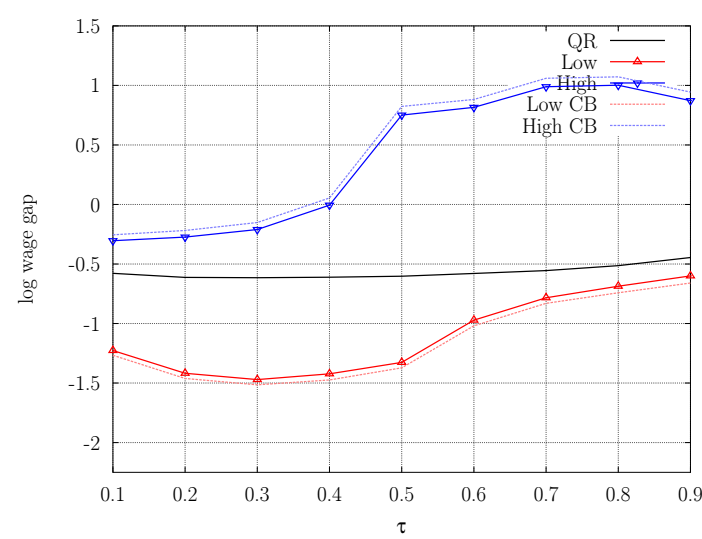

1995-1999

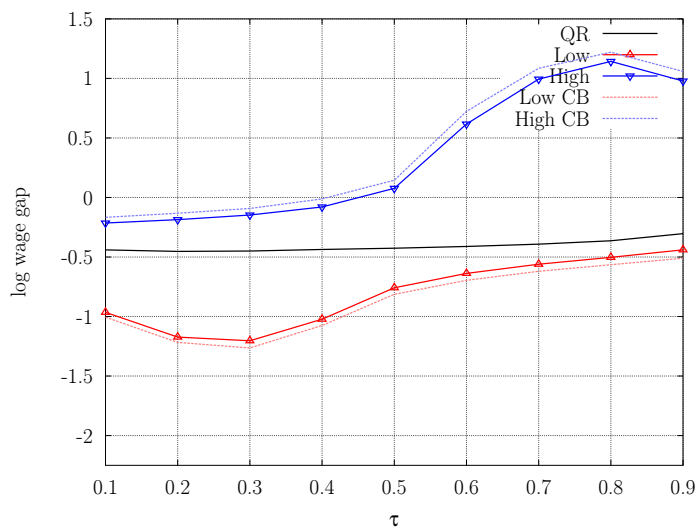

Estimated quantile gender wage gap (female - male) conditional on having 12 or fewer years of education with average characteristics. The solid black line shows the quantile gender wage gap when selection is ignored. The blue and red lines with upward and downward pointing triangles show upper and lower bounds that account for employment selection for females. The dashed lines represent a uniform $90 \%$ confidence region for the bounds.

Figure C.4. Quantile bounds for $\geq 16$ years of education and single 1975-1979

1995-1999
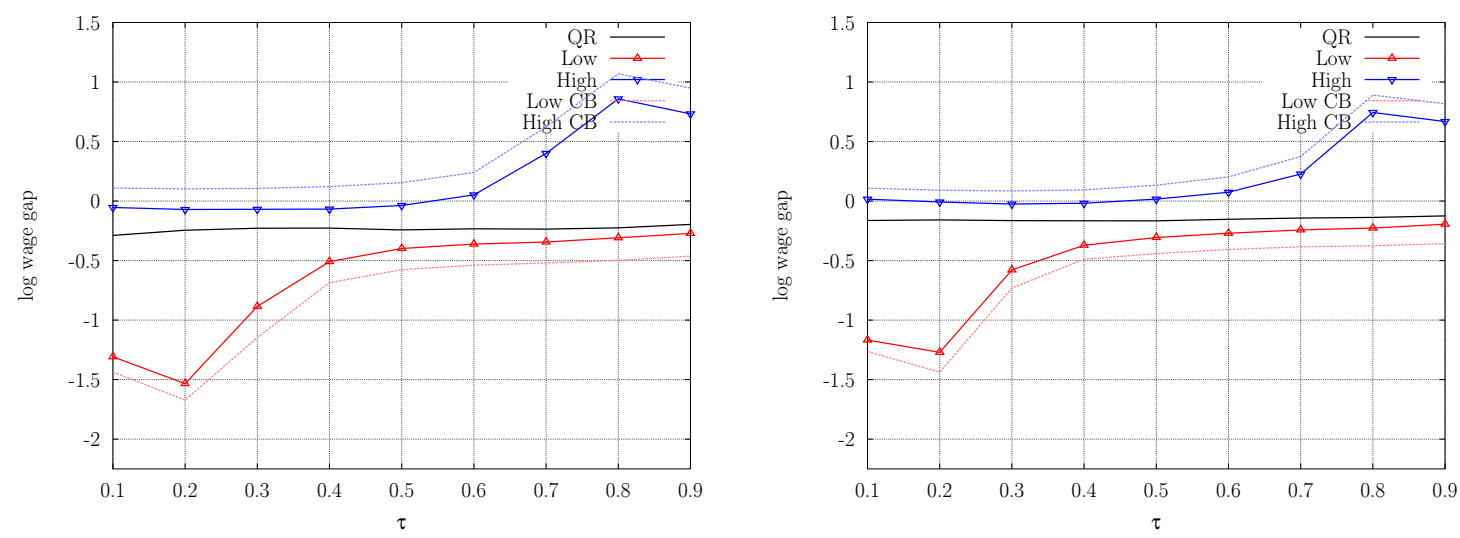

Estimated quantile gender wage gap (female - male) conditional on being single with at least 16 years of education and average characteristics. The solid black line shows the quantile gender wage gap when selection is ignored. The blue and red lines with upward and downward pointing triangles show upper and lower bounds that account for employment selection for females. The dashed lines represent a uniform $90 \%$ confidence region for the bounds. 
Figure C.5. Quantile bounds for single and $\geq 16$ years of education imposing stochastic dominance

1975-1979

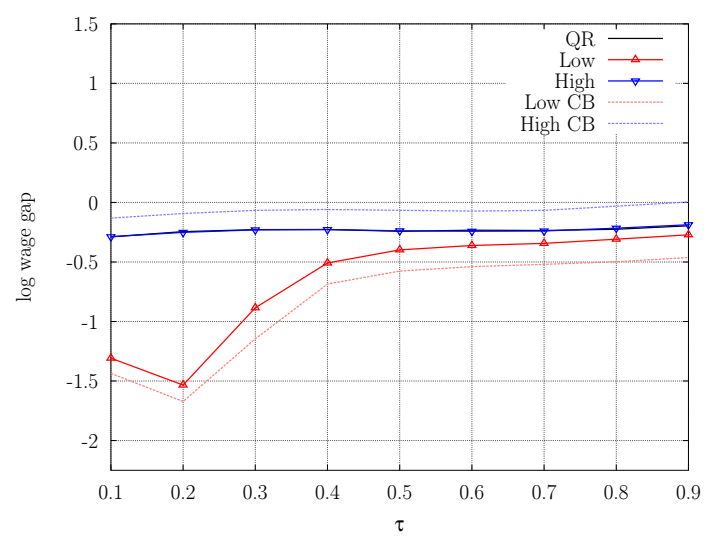

1995-1999

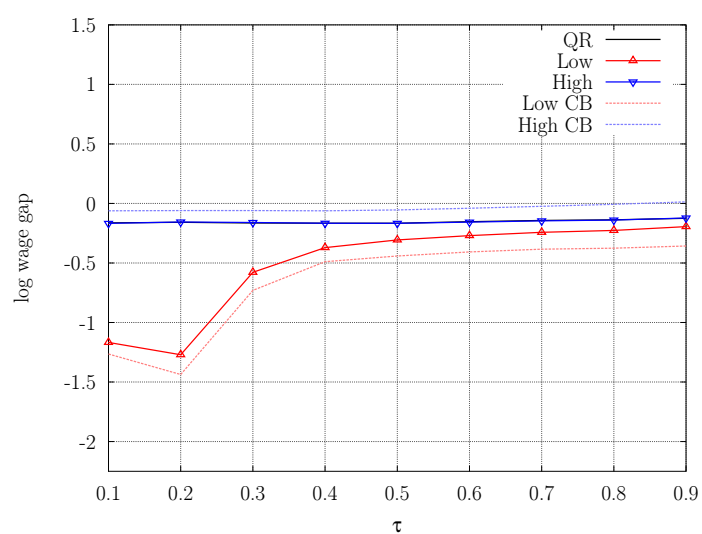

Estimated quantile gender wage (female - male) conditional on average characteristics. The solid black line shows the quantile gender wage when selection is ignored. The blue and red lines with upward and downward pointing triangles show upper and lower bounds that account for employment selection for females. The dashed lines represent a uniform $90 \%$ confidence region for the bounds.

Figure C.6. Quantile bounds for single and $\geq 16$ years of education imposing the median restriction

1975-1979

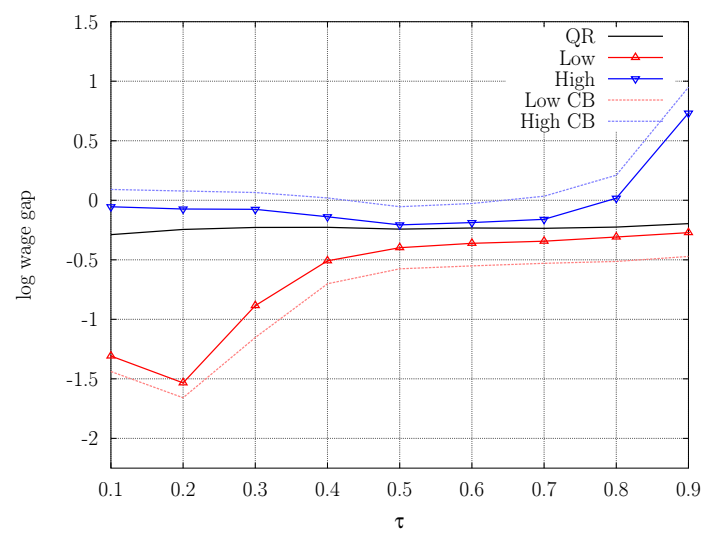

1995-1999

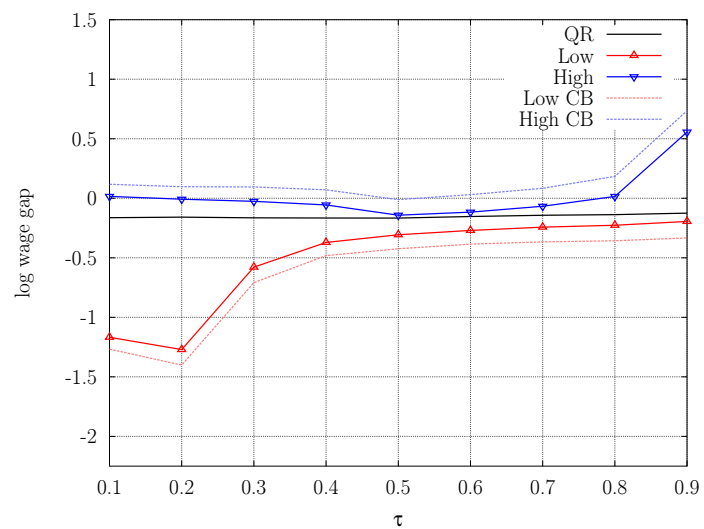

Estimated quantile gender wage (female - male) conditional on average characteristics. The solid black line shows the quantile gender wage when selection is ignored. The blue and red lines with upward and downward pointing triangles show upper and lower bounds that account for employment selection for females. The dashed lines represent a uniform $90 \%$ confidence region for the bounds. 


\section{REFERENCES}

Andrews, D. W. K. (1994): Empirical Process Methods in Econometricsvol. IV of Handbook of Econometrics, pp. 2248-2294.

Andrews, D. W. K., AND P. J. BArwick (2012): "Inference for Parameters Defined by Moment Inequalities: A Recommended Moment Selection Procedure," Econometrica, 80(6), 2805-2826.

Andrews, D. W. K., AND X. Shi (2013): "Inference Based on Conditional Moment Inequalities," Econometrica, 81, 609-666.

Andrews, D. W. K., And G. Soares (2010): "Inference for Parameters Defined by Moment Inequalities Using Generalized Moment Selection," Econometrica, 78, 119-157.

Artstein, Z., AND R. A. Vitale (1975): "A Strong Law of Large Numbers for Random Compact Sets," The Annals of Probability, 3(5), 879-882.

Belloni, A., And V. Chernozhukov (2009a): "On the computational complexity of MCMC-based estimators in large samples," The Annals of Statistics, 37(4), 2011-2055.

(2009b): "Post-L1-Penalized Estimators in High-Dimensional Linear Regression Models," arXiv:1001.0188.

Belloni, A., V. Chernozhukov, D. Chetverikov, and I. Fernandez-Val (2018): "Conditional Quantile Processes based on Series or Many Regressors," Journal of Econometrics, forthcoming.

Beresteanu, A., AND F. Molinari (2008): "Asymptotic Properties for a Class of Partially Identified Models," Econometrica, 76(4), 763-814.

Blau, F. D., AND L. M. Kahn (1997): "Swimming Upstream: Trends in the Gender Wage Differential in the 1980s," Journal of Labor Economics, 15, 1-42.

(2017): "The Gender Wage Gap: Extent, Trends, and Explanations," Journal of Economic Literature, 55, 789-865.

Blundell, R., A. Gosling, H. Ichimura, And C. Meghir (2007): "Changes in the Distribution of Male and Female Wages Accounting for Employment Composition Using Bounds," Econometrica, 75(2), 323-363.

Bontemps, C., T. Magnac, And E. Maurin (2012): "Set Identified Linear Models," Econometrica, 80, 1129-1155.

Bugni, F. A. (2010): "Bootstrap Inference in Partially Identified Models Defined by Moment Inequalities: Coverage of the Identified Set," Econometrica, 78, 735-753.

Canay, I. A. (2010): "EL Inference for Partially Identified Models: Large Deviations Optimality and Bootstrap Validity," Journal of Econometrics, 156, 408-425.

CARD, D. (1999): The Causal Effect of Education on Earningschap. 30, pp. 1801-1863. Elsevier.

CARD, D., AND J. E. DiNARdo (2002): "Skill Biased Technological Change and Rising Wage Inequality: Some Problems and Puzzles," Journal of Labor Economics, 20, 733-783.

Chen, X. (2007): "Large Sample Sieve Estimation of Semi-Nonparametric Models," Handbook of Econometrics, 6 .

Chernozhukov, V., H. Hong, And E. Tamer (2007): "Estimation and Confidence Regions for Parameter Sets in Econometric Models," Econometrica, 75(5), 1243-1284.

Chernozhukov, V., E. Kocatulum, And K. Menzel (2015): "Inference on sets in finance," Quantitative Economics, 6, 309-358.

Chernozhukov, V., S. Lee, And A. Rosen (2013): "Intersection Bounds: Estimation and Inference," Econometrica, 81, 667-737.

Davydov, Y. A., M. A. Lifshits, And N. V. Smorodina (1998): Local Properties of Distribuions of Stochastic Functionals. American Mathematical Society.

Einav, L., A. Finkelstein, S. P. Ryan, P. Schrimpf, And M. R. Cullen (2013): "Selection on Moral Hazard in Health Insurance," American Economic Review, 103(1), 178-219.

FAnG, Z., AND A. SAntos (2018): "Inference on Directionally Differentiable Functions," Review of Economic Studies, forthcoming.

Foresi, S., AND F. Peracchi (1995): "The Conditional Distribution of Excess Returns: An Empirical Analysis," Journal of the American Statistical Association, 90, 451-466. 
Galichon, A., AND M. Henry (2009): "A test of non-identifying restrictions and confidence regions for partially identified parameters," Journal of Econometrics, 152(2), 186-196.

Ghosal, S., A. Sen, And A. W. van Der VaArt (2000): "Testing Monotonicity of Regression," Annals of Statistics, 28, 1054-1082.

Han, A., AND J. A. Hausman (1990): "Flexible Parametric Estimation of Duration and Competing Risk Models," Journal of Applied Econometrics, 5, 1-28.

He, X., AND Q.-M. Shao (2000): "On Parameters of Increasing Dimensions," Journal of Multivariate Analysis, 73(1), 120-135.

Hirano, K., G. Imbens, AND G. Ridder (2003): "Efficient estimation of average treatment effects using the estimated propensity score," Econometrica, 71(4), 1161-1189.

Imbens, G. W., And C. F. Manski (2004): "Confidence Intervals for Partially Identified Parameters," Econometrica, 72, 1845-1857.

Imbens, G. W., AND J. M. WooldRIDGe (2009): "Recent developments in the econometrics of program evaluation," Journal of Economic Literature, 47, 5-86.

Jasso, G., And M. R. Rosenzweig (2008): "Selection Criteria and the Skill Composition of Immigrants: A Comparative Analysis of Australian and U.S. Employment Immigration," DP 3564, IZA.

Juster, F. T., AND R. Suzman (1995): "An Overview of the Health and Retirement Study," Journal of Human Resources, 30 (Supplement), S7-S56.

KAIDO, H. (2016): "A Dual Approach to Inference for Partially Identified Econometric Models," Journal of Econometrics, 192, 269-290.

Kaido, H., AND A. Santos (2014): "Asymptotically Efficient Estimation of Models Defined by Convex Moment Inequalities," Econometrica, 82, 387-413.

Kline, P., And A. Santos (2013): "Sensitivity to Missing Data Assumptions: Theory and An Evaluation of the U.S. Wage Structure," Quantitative Economics, 4, 231-267.

Lorentz, G. G. (1986): Approximation of Functions. Chelsea.

Lust-Piquard, F., AND G. Pisier (1991): "Non commutative Khintchine and Paley inequalities," Arkiv för Matematik, 29(1), 241-260.

Magnac, T., And E. Maurin (2008): "Partial Identification in Monotone Binary Models: Discrete Regressors and Interval Data," Review of Economic Studies, 75(3), 835-864.

Manski, C. F. (1994): The selection problemchap. 4, pp. 143-170, Advances in Econometrics, Sixth World Congress. Cambridge University Press.

- (2003): Partial Identification of Probability Distributions. Springer Verlag, New York. (2007): Identification for Prediction and Decision. Harvard University Press, Cambridge, MA.

Manski, C. F., AND E. TAMER (2002): "Inference on Regressions with Interval Data on a Regressor or Outcome," Econometrica, 70(2), 519-546, ArticleType: primary_article / Full publication date: Mar., 2002 / Copyright (C) 2002 The Econometric Society.

Molchanov, I. (2005): Theory of Random Sets. Springer Verlag, London.

Mulligan, C. B., AND Y. Rubinstein (2008): "Selection, Investment, and Women's Relative Wages Over Time," Quarterly Journal of Economics, 123(3), 1061-1110.

NEWEy, W. K. (1997): "Convergence rates and asymptotic normality for series estimators," Journal of Econometrics, 79(1), 147-168.

Olley, G. S., AND A. PAKes (1996): "The Dynamics of Productivity in the Telecommunications Equipment Industry," Econometrica, 64(6), 1263-1297, ArticleType: primary_article / Full publication date: Nov., 1996 / Copyright 1996 The Econometric Society.

Picketty, T. (2005): "Top Income Shares in the Long Run: An Overview," Journal of the European Economic Association, 3, 382-392.

Pollard, D. (2002): A User's Guide to Measure Theoretic Probability. Cambridge.

Rockafellar, R. (1970): Convex Analysis. Princeton University Press.

Romano, J. P., AND A. M. Shaikh (2008): "Inference for identifiable parameters in partially identified econometric models," Journal of Statistical Planning and Inference, 138(9), 2786-2807.

(2010): "Inference for the Identified Set in Partially Identified Econometric Models," Econometrica, 78, 169-211. 
Rosen, A. M. (2008): "Confidence sets for partially identified parameters that satisfy a finite number of moment inequalities," Journal of Econometrics, 146(1), 107-117.

Rudelson, M. (1999): "Random vectors in the isotropic position," Journal of Functional Analysis, 164(1), $60-72$.

Schneider, R. (1993): Convex Bodies: The Brunn-Minkowski Theory, Encyclopedia of Mathematics and Its Applications. Cambridge University Press, Cambridge, UK.

Stoye, J. (2007): "Bounds on Generalized Linear Predictors with Partially Identified Outcomes," Reliable Computing, 13, 293-302.

1299-1315.

Townsend, R. M., AND S. S. URzuA (2009): "Measuring the Impact of Financial Intermediation: Linking Contract Theory to Econometric Policy Evaluation," Macroeconomic Dynamics, 13(Supplement S2), 268316.

VAN DER VAART, A. W. (2000): Asymptotic statistics. Cambridge University Press.

VAN DER VAART, A. W., AND J. Wellner (1996): Weak Convergence and Empirical Processes: With Applications to Statistics. Springer.

Vitale, R. A. (1985): " $L_{p}$ Metric for Compact, Convex Sets," Journal of Approximation Theory, 45, $280-287$.

Department of Economics, Stanford University \& Microsoft Research New England

Department of Economics, M. I. T.

Department of Economics, Cornell University

Vancouver School of Economics, University of British Columbia 